\title{
CAKE DIVISION BY MAJORITY DECISION
}

\author{
HANS GERSBACH \\ BERNHARD PACHL
}

CESIFO WORKING PAPER NO. 1872

CATEgORY 2: Public CHOICE

DECEMBER 2006

An electronic version of the paper may be downloaded

- from the SSRN website:

www.SSRN.com

- from the RePEc website:

www.RePEc.org

- from the CESifo website:

www.CESifo-group.de 


\title{
CAKE DIVISION BY MAJORITY DECISION
}

\begin{abstract}
We consider a collective choice process where three players make proposals sequentially on how to divide a given quantity of resources. Afterwards, one of the proposals is chosen by majority decision. If no proposal obtains a majority, a proposal is drawn by lot. We establish the existence of the set of subgame perfect equilibria, using a suitable refinement concept. In any equilibrium, the first agent offers the whole cake to the second proposal-maker, who in turn offers the whole cake back to the first agent. The third agent is then indifferent about dividing the cake between himself and the first or the second agent.
\end{abstract}

JEL Code: C72, D30, D39, D72.

Keywords: division of a cake, majority decisions, tie-breaking rules.

Hans Gersbach
Center of Economic Research
at ETH Zurich
Zürichbergstr. 18
8092 Zurich
Switzerland
hgersbach@ethz.ch

Hans Gersbach

at ETH Zurich

8092 Zurich

gersbach@ethz.ch

\author{
Bernhard Pachl \\ Department of Economics \\ University of Heidelberg \\ Grabengasse 14 \\ 69117 Heidelberg \\ Germany \\ Bernhard.Pachl@urz.uni-heidelberg.de
}

This version: November 2006

We have received extremely valuable comments from a referee. We also wish to thank Volker Hahn, Noemi Hummel, Achim Schniewind, Till Requate and seminar participants in Heidelberg and Virginia for helpful comments. Financial support from the "Deutsche Forschungsgemeinschaft (DFG)" is gratefully acknowledged. 


\section{Introduction}

In this paper, we study the division of a cake by majority decision with lotteries to break ties. Each of three players proposes a division of the cake. Afterwards, they choose one of the proposals by majority voting. If no proposal receives a majority, one of the proposals is chosen by lottery. The essential features of this model are that there is a definite end of the collective choice process and that no resources are discarded in case of disagreement.

There is a number of real-world examples in which proposals are made sequentially and voting takes place after all the proposals have been made. For instance, in legislative bargaining with simple open rules, agents are appointed sequentially for agenda-setting and they can make proposals, knowing the proposals made so far. If an agent brings a set of proposals to vote and one proposal passes, the process ends [see Krehbiel (1991) for a survey of such legislative organizations]. Moreover, many collective decisions by committees of public or private organizations are governed by open sequential proposals followed by a majority vote. The lottery rule for tie-breaking is somewhat less common, although there is a number of real-world substitutes more or less resembling a lottery. In some legislatures, for instance, the chairman of a committee does not participate in the voting itself, but he has a casting vote allowing him to break ties. If his preferences are not known to the committee members, to them, his tie-breaking move may be similar to a lottery. One can experience such decision schemes personally in committee meetings on budget allocations at German universities.

We show here that there is an infinite number of subgame perfect equilibria that all yield the same two outcomes. In both outcomes, the first player making a proposal offers the whole cake to the second proposal-maker, who in turn offers the whole cake back to the first player. The difference between the two outcomes is as follows: The third player offers half of the cake to himself in both outcomes, while he offers the other half to the first player in one outcome and to the second player in the other. The proposal will be chosen by a majority in the voting stage. Hence, in both outcomes, the cake will merely be divided among 2 players. Player 3 is always one of the two players receiving half of the cake.

Player 3 is in a dominant position because given any two former proposals, he can decide which proposal should get a majority by making an adequate proposal himself. Since Player 2 is aware of this, he will always try to design his proposal in such a 
way that Player 3 will choose him as a partner, thus getting more than Player 1. Ultimately, this is the reason why Player 1 tries to disadvantage Player 2 as much as possible by offering the whole cake to Player 2, because then, the only chance Player 2 has of countering this proposal is to offer the whole cake to Player 1 as well. This competition results in symmetric disadvantages for Players 1 and 2 and it allows Player 3 to choose a partner at random. Player 3 is indifferent about whom to cooperate with because he has to offer both players the same utility, compensating one of them for a tie and drawing him into a coalition. Therefore, any mixed strategy by Player 3, to offer half of the cake to Player 1 or 2, can be played in equilibrium with some probability.

\section{Relation to the Literature}

Majority rule and drawing lots are standard procedures for dividing resources in collective choice processes of the kind encountered in legislatures or committees [e.g. Baron and Ferejohn (1989), Bernholz and Breyer (1994)]. Accordingly, we are interested in the positive analysis of the division of resources we obtain in such cases. Our work is related to two strands of literature. First, the division of resources has been studied from the perspective of alternative collective choice processes. Mueller (1978) examined the veto rule, under which the resources are thrown away in case in disagreement. In such games, equilibria show a strong tendency towards equal shares for each individual. Our analysis deals with majority decisions and takes the view that resources are not thrown away in case of disagreement, but are subject to a tie-breaking procedure.

Second, Baron and Ferejohn (1989) have examined the division of resources by majority rule. If no agreement is reached, players can make new proposals. The proposer receives disproportionate benefits and the number of recipients of positive shares of the cake is at least a bare majority, but may also exceed this figure. In our case, there is a definite end to the collective choice process, which forces players to choose between agreement and tie-breaking procedure.

\section{The Game}

We consider a game involving three players denoted by $i, j$, and $k=1,2,3$ who wish to divide a cake in the following way:

First, at the proposal stage, the players sequentially make open proposals about the 
division of the cake.

1. Player 1 proposes a division of the cake to Players 2 and 3, not knowing their proposals.

2. Player 2 makes a second proposal, knowing Player 1's proposal.

3. Player 3 suggests a division, knowing the proposals of the two former players.

Then, at the voting stage, the group selects one of these proposals ${ }^{1}$ by majority voting. An offer receiving 2 or 3 votes will be implemented. If no majority can be reached, i.e. if each proposal gets one vote, ${ }^{2}$ the winning proposal is selected by drawing lots and each proposal is selected with a probability of $\frac{1}{3}$. Hence, the cake is either divided by majority decision or by lot.

Note that without loss of generality, the labeling of the players and hence the sequence of proposals by the players is given exogenously. ${ }^{3}$

A proposal by Player $i$ is denoted by $D_{i}=\left(a_{i 1}, a_{i 2}, a_{i 3}\right)$. Accordingly, $a_{i 1}, a_{i 2}$, and $a_{i 3}$ denote the shares of the cake offered by Player $i$ to Players 1, 2, and 3 respectively. The resource constraint implies that $\sum_{j=1}^{3} a_{i j}=1, \forall i$. Thus, after every player has made his proposal, we obtain

$$
\begin{aligned}
& D_{1}=\left(a_{11}, a_{12}, a_{13}\right) \\
& D_{2}=\left(a_{21}, a_{22}, a_{23}\right) \\
& D_{3}=\left(a_{31}, a_{32}, a_{33}\right)
\end{aligned}
$$

To simplify our presentation, we rewrite the complete set of three proposals as the matrix

$$
A=\left(\begin{array}{lll}
a_{11} & a_{12} & a_{13} \\
a_{21} & a_{22} & a_{23} \\
a_{31} & a_{32} & a_{33}
\end{array}\right)
$$

Every player is risk-neutral and individual $j$ derives utility ${ }^{4}$

$$
U:[0,1] \rightarrow[0,1], U\left(a_{i j}\right)=a_{i j}
$$

\footnotetext{
${ }^{1}$ If two proposals coincide, they are treated as one proposal.

${ }^{2}$ The omission of abstentions will be explained in the characterization of equilibria.

${ }^{3}$ We might add a pre-stage at which the label of an agent is selected at random. Such an additional stage would equalize expected utilities across players in case of identical utility functions. Since we are focusing on actual resource divisions, we skip the pre-stage.

${ }^{4}$ Since all players are identical, we drop the index for the utility function.
} 
if the proposal $D_{i}$ is selected by majority voting. The expected utility $u_{j}$ for Player $j$ in the case of drawing lots is then given by

$$
u_{j}=\frac{1}{3} \sum_{i=1}^{3} a_{i j}, j=1,2,3
$$

In many parts of the analysis, we need the relative quality of the offers made to the specific players. Therefore, we introduce the rank matrix $R$. In this matrix, the best offer (the biggest share of the cake) $a_{i j}$ for Player $j$ is labeled 3, the second best 2 , and the worst 1. If two or more offers are identical, they have the same rank. For example the proposal matrices

$$
A_{1}=\left(\begin{array}{lll}
0.3 & 0.2 & 0.5 \\
0.1 & 0.7 & 0.2 \\
0.6 & 0.3 & 0.1
\end{array}\right) A_{2}=\left(\begin{array}{ccc}
0.3 & 0.6 & 0.1 \\
0.4 & 0.5 & 0.1 \\
0.4 & 0.5 & 0.1
\end{array}\right)
$$

then convert into the rank matrices

$$
R_{1}=\left(\begin{array}{lll}
2 & 1 & 3 \\
1 & 3 & 2 \\
3 & 2 & 1
\end{array}\right) R_{2}=\left(\begin{array}{lll}
1 & 3 & 3 \\
3 & 2 & 3 \\
3 & 2 & 3
\end{array}\right)
$$

Additionally, we denote the biggest share $a_{i j}$ of the cake offered to Player $j$ by $X_{j}$, the second biggest by $Y_{j}$, and the worst by $Z_{j}$.

If we label the entries of $R$ as $r_{i j}(i, j=1,2,3)$, where $r_{i j}$ is the rank of Player $i$ 's proposal made to Player $j$, then the conversion function $\Phi[A]=R$ is technically given by

$$
\Phi: r_{i j}=\sum_{k=1}^{3} \Theta\left(a_{i j}-a_{k j}\right)
$$

where $\Theta(p-q)(p, q \in \mathbb{R})$ is the Heavyside function given by

$$
\Theta(p-q)= \begin{cases}1 & p-q \geq 0 \\ 0 & \text { otherwise }\end{cases}
$$

\section{Voting Equilibria and Proposal-Making}

In this section, we first derive the voting equilibria based on a given set of proposals. The voting strategy of Player $i$ is to select one proposal. ${ }^{5}$ We note that the concept

\footnotetext{
${ }^{5}$ Allowing abstention does not change the voting equilibria. Abstention is weakly dominated by voting for the best proposal for Player $i$.
} 
of a Nash equilibrium is insufficient for voting games with non-unanimity voting rules, as it can involve weakly dominated strategies. For that purpose, we use the following refinements and tie-breaking rules (for cases of indifference between payoffs). These hold throughout the paper.

\subsection{Refinements}

\section{Refinement 1}

A Nash equilibrium of the voting game has to be trembling hand perfect. ${ }^{6}$

Trembling hand perfection has two immediate consequences:

\section{Lemma 1}

Suppose that refinement 1 holds. Then

(i) A player never votes for the least favorable proposal made to him, i.e. a proposal labeled 1 in the rank matrix.

(ii) In any Nash equilibrium where agents $i$ and $j(i \neq j)$ vote for the same proposal, agent $k(k \neq i, j)$ votes for his best proposal.

The elimination of weakly dominated strategies is standard. The second property also follows directly from the definition of trembling hand perfection, as any error by Players $i$ or $j$ leads either to a tie-break or to a majority win for the best proposal of voter $i$.

The next refinement eliminates voting equilibria that are payoff-dominated.

\section{Refinement 2}

If only one proposal includes 2 maxima, then it is the unique equilibrium.

This property follows from payoff dominance (see Fudenberg and Tirole 1992). A proposal with 2 maxima for, say, individuals $i$ and $j$, is a Nash equilibrium supported by the votes of $i$ and $j$. Any other possible Nash equilibrium is worse for $i$ and $j$ and hence payoff-dominated for the coalition $\{i, j\}$.

Even if both refinements are applied, we will still have multiple equilibria. For that purpose, we use the notion of correlated equilibria with public randomization introduced by Aumann (1974) (see e.g. Myerson 1991 for discussion). This concept assumes that voters engage in pre-play communication and use a coordination device to settle for

\footnotetext{
${ }^{6}$ See Selten (1975) for the original formalization and Fudenberg and Tirole (1992) for a survey.
} 
a particular equilibrium. Such a device is a publicly observable random variable that agents use to determine which equilibrium should be played. For instance, agents may flip a coin, or a mediator may announce the outcome of the randomization process.

This is illustrated in the following example:

$$
A=\left(\begin{array}{lll}
0.6 & 0.0 & 0.4 \\
0.4 & 0.1 & 0.5 \\
0.2 & 0.8 & 0.0
\end{array}\right) \longrightarrow R=\left(\begin{array}{lll}
3 & 1 & 2 \\
2 & 2 & 3 \\
1 & 3 & 1
\end{array}\right)
$$

In this case, $D_{1}$ and $D_{2}$ are equilibria and Player 1 and Player 3 prefer their second-best offer to a tie-break. Furthermore, these proposals contain the first- and second-best offer by Player 1 and Player 3. Therefore, we assume that Player 1 and Player 3 are playing a correlated strategy. With probability $p_{1}=\frac{1}{2}$, both play $D_{1}$, and with $p_{2}=\frac{1}{2}$ they play $D_{2}$.

Accordingly, we use the concept of correlated equilibria as follows:

\section{Refinement 3}

A correlated equilibrium arises if two proposals $D_{i}, D_{j}(i \neq j, i, j=1,2,3)$ are equilibria ${ }^{7}$ and they contain the best and second-best offer by the same two players. In the correlated equilibrium, both players under consideration will vote with probability $\frac{1}{2}$ for $D_{i}$ or $D_{j}$. The expected payoffs for all players are then given by $C_{i j}=\frac{1}{2}\left(D_{i}+D_{j}\right)$.

Note that correlated equilibria cannot be used in particular cases, e.g. when two equilibria occur:

$$
A=\left(\begin{array}{lll}
0.0 & 0.5 & 0.4 \\
0.4 & 0.1 & 0.5 \\
0.1 & 0.3 & 0.6
\end{array}\right) \longrightarrow R=\left(\begin{array}{lll}
1 & 3 & 1 \\
3 & 1 & 2 \\
2 & 2 & 3
\end{array}\right)
$$

Here, $D_{2}$ and $D_{3}$ are equilibria, but Player 2, who is needed for the majority of $D_{3}$, has no incentive to establish a coordination for $D_{2}$ and $D_{3}$ because $D_{3}$ contains his second-best and $D_{2}$ his worst offer. These cases are discussed in subsection 4.3 and ruled out by Refinement 4 .

\subsection{Tie-breaking Rules}

In this subsection, we introduce some tie-breaking rules to simplify the exposition. Since agents try to receive a share as big as possible, there is only one possible motive

\footnotetext{
${ }^{7}$ We can neglect correlated equilibria with public randomization over all three proposals, as they are equivalent to selecting proposals by lot.
} 
for an agent to deviate from voting for the best proposal and to vote for the secondbest offer instead: If he cannot establish a majority of votes for his best proposal, the player will prefer his second-best offer to a tie-break. To illustrate this case, consider the following example involving the proposal matrix:

$$
A=\left(\begin{array}{lll}
0.7 & 0.0 & 0.3 \\
0.5 & 0.5 & 0.0 \\
0.0 & 0.1 & 0.9
\end{array}\right) \longrightarrow R=\left(\begin{array}{lll}
3 & 1 & 2 \\
2 & 3 & 1 \\
1 & 2 & 3
\end{array}\right)
$$

Because of Refinement 1, Player 2 votes for $D_{2}$ and Player 3 for $D_{3}$. $D_{1}$, favored by Player 1, thus has no chance of becoming selected, but $Y_{1}=\frac{1}{2}$ is greater than $u_{1}=\frac{1}{3}\left(\frac{7}{10}+\frac{1}{2}+0\right)=\frac{2}{5}$, his expected share in the case of drawing lots. Therefore, Player 1 votes for $D_{2}$ together with Player 2 to avoid a tie-break decision. The case where the payoffs of the second-best offer and of drawing lots coincide is resolved in the following tie-breaking rule:

\section{Tie-Break Rule 1}

If $Y_{j}=u_{j}$, all players will prefer their second-best bid to drawing lots.

Note that "prefer" in tie-breaking rule 1 means that Player $i$ will avoid drawing lots if he is indifferent between his second-best proposal and drawing lots. The tie-breaking rule means that Player $i$ will vote for his best proposal if it receives a majority. Otherwise, he will vote for the second-best proposal. The tie-breaking rule immediately implies

\section{Lemma 2}

Suppose that there exists at least one voting equilibrium where a majority is formed. Then drawing lots will not occur as an equilibrium.

To formulate the next tie-breaking rule, we denote an equilibrium as a single-proposal equilibrium if that single proposal is selected without the use of random selection devices. A single-proposal equilibrium necessarily requires that pure voting strategies are played and that a majority supports one proposal.

\section{Tie-Break Rule 2}

If the payoffs coincide, a player will prefer a single-proposal equilibrium to a correlated equilibrium and a correlated equilibrium to drawing lots.

\subsection{Proposal-Making}

Having characterized the structure of the voting equilibria, we turn now to proposalmaking. In formulating the proposal-making stage, we face two problems. 
First, multiple voting equilibria may still exist (see e.g. the proposal matrix in equation 9). Second, players may be indifferent between several proposals (e.g. Player 3 is always indifferent between at least two proposals if $D_{1}=\left(a_{11}, 1-a_{11}, 0\right)$ and $\left.D_{2}=\left(1-a_{11}, a_{11}, 0\right)\right)$.

Addressing the first point, we now introduce another refinement.

\section{Refinement 4}

Given the proposals $D_{1}$ and $D_{2}$, Player 3 makes his proposal in such a way that the proposal matrix $A$ exhibits either a single-proposal equilibrium, a correlated equilibrium, or an equilibrium with drawing lots.

This refinement ensures that payoffs are well-defined at the voting stage. This can be justified by the aversion against strategic uncertainty. A priori, it is unclear whether Player 3 can always choose among the three options in the refinement. In the proof of the overall equilibrium, we will show that this is always possible for Player 3.

The second point is handled by an additional tie-breaking rule.

\section{Tie-Break Rule 3}

If Player $i$ is indifferent between making several proposals, every proposal is submitted with the same probability.

\subsection{The Structure of Voting Equilibria}

After these preparations, we can now provide an overview of the structure of voting equilibria. We start by calculating equilibria in two examples. For this purpose, we extend the conversion of proposal matrix $A$ to rank matrix $R$, since it is often necessary to distinguish whether it is possible for a player to deviate to his second-best proposal or not. This is done by introducing rank $2^{*}$ if any $Y_{j} \geq u_{j}(j=1,2,3)$. If we consider the example given in (10), we get

$$
A=\left(\begin{array}{lll}
0.7 & 0.0 & 0.3 \\
0.5 & 0.5 & 0.0 \\
0.0 & 0.1 & 0.9
\end{array}\right) \longrightarrow R=\left(\begin{array}{lll}
3 & 1 & 2 \\
2^{*} & 3 & 1 \\
1 & 2 & 3
\end{array}\right)
$$

To calculate the equilibria, we ask whether Player $i$ deviates, given the votes of Players $j$ and $k\left(i \neq j \neq k\right.$ and the players are labeled $P_{1}, P_{2}$ and $\left.P_{3}\right)$. If Player $i$ does deviate, his payoff is underlined. 


\begin{tabular}{|c|c|c|c|}
\hline \multicolumn{4}{|c|}{$P_{1} \longrightarrow D_{1}$} \\
\hline$P_{2}{ }^{P_{3}}$ & $D_{1}$ & $D_{2}$ & $D_{3}$ \\
\hline$D_{1}$ & $\frac{0.7}{0.0}$ & $\frac{0.7}{0_{0.0}}$ & $\frac{0.7}{0.0}$ \\
\hline$D$ & $\underline{\underline{0.7}} \underline{\underline{0.0}}$ & $\frac{0.5}{\underline{0.5}}$ & 0.4 \\
\hline & 0.7 & 0. & \\
\hline
\end{tabular}

\begin{tabular}{|c|c|c|c|}
\hline \multicolumn{4}{|c|}{$P_{1} \longrightarrow D_{2}$} \\
\hline$P_{2}^{P_{3}}$ & $D_{1}$ & $D_{2}$ & $D_{3}$ \\
\hline$D_{1}$ & $\begin{array}{ll}0.7 & \\
& 0.0\end{array}$ & & \\
\hline$D$ & $\begin{array}{l}0.5 \\
\quad \underline{0.5}\end{array}$ & & $\begin{array}{c}\underline{0.5} \\
\underline{0.5}\end{array}$ \\
\hline$D$ & & & \\
\hline
\end{tabular}

\begin{tabular}{|c|c|c|c|}
\hline \multicolumn{4}{|c|}{$P_{1} \longrightarrow D_{3}$} \\
\hline & $D_{1}$ & $D_{2}$ & $D$ \\
\hline$D_{1}$ & $\begin{array}{l}0.7 \\
\quad 0.0\end{array}$ & & \\
\hline & $\begin{array}{l}0.4 \\
\quad \underline{0.2} \\
0\end{array}$ & $\begin{array}{l}0.5 \\
\quad 0 .\end{array}$ & $\begin{array}{l}0.0 \\
0 . \\
\end{array}$ \\
\hline & & & \\
\hline
\end{tabular}

In this case, the only equilibrium voting scheme is

$$
\left.\begin{array}{l}
P_{1} \longrightarrow D_{2} \\
P_{2} \longrightarrow D_{2} \\
P_{3} \longrightarrow D_{3}
\end{array}\right\} \Longrightarrow D_{2} \text { is chosen }
$$

We see that these calculations can be done simply by regarding the extended rank matrix. For this purpose, we look at the example given in (8). We obtain the extended rank matrix

$$
A=\left(\begin{array}{lll}
0.6 & 0.0 & 0.4 \\
0.4 & 0.1 & 0.5 \\
0.2 & 0.8 & 0.0
\end{array}\right) \longrightarrow R=\left(\begin{array}{lll}
3 & 1 & 2^{*} \\
2^{*} & 2 & 3 \\
1 & 3 & 1
\end{array}\right)
$$

and end up with

\begin{tabular}{|c|c|c|c|}
\hline & $\begin{array}{l}P_{1} \\
D_{1}\end{array}$ & ] & \\
\hline$P_{1}$ & & & \\
\hline & $\begin{array}{r}2 \\
{ }_{3} \\
\end{array}$ & ${ }^{2}$ & ${ }^{2}$ \\
\hline & $\underline{\underline{T}}_{T}$ & & $\underline{3}$ \\
\hline
\end{tabular}

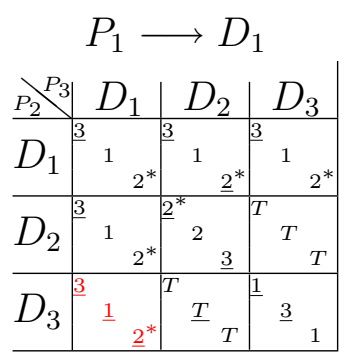

\begin{tabular}{|c|c|c|c|}
\hline \multicolumn{3}{|c|}{$P_{1} \longrightarrow D_{3}$} & $D_{3}$ \\
\hline 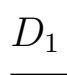 & & & 3 \\
\hline$D_{2}$ & $T_{T}$ & 2 & 3 \\
\hline & $\underline{3}_{1}$ & $\underline{3}$ & $\underline{3}$ \\
\hline
\end{tabular}

Now, two voting schemes are equilibria.

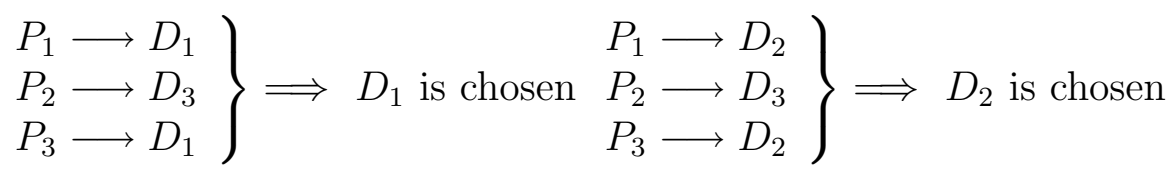

Since players 1 and 3 form the majority in both voting schemes, these equilibria are correlated.

In the following, we discuss the possibility of extending rank matrices and their equilibria. We denote a single-proposal equilibrium as $D_{i}^{*}$, an equilibrium with drawing lots as $T^{*}$ and a correlated equilibrium as $C_{i j}^{*}(i \neq j)$. In the discussion, the matrices 
described are representatives of a whole class of matrices that can be derived from the given matrix by interchanging columns and rows, or two entries.

For example

$$
\left(\begin{array}{lll}
1 & 3 & 2 \\
2 & 1 & 3 \\
3 & 2 & 1
\end{array}\right)
$$

is, among other things, a representative of

$$
\left(\begin{array}{lll}
2 & 1 & 3 \\
1 & 3 & 2 \\
3 & 2 & 1
\end{array}\right) \text { and }\left(\begin{array}{lll}
2 & 3 & 2 \\
1 & 1 & 3 \\
3 & 2 & 1
\end{array}\right)
$$

Where convenient, we omit cases with $X_{i} \neq Y_{i}=Z_{i}$, because they are qualitatively equal to cases with $X_{i} \neq Y_{i} \neq Z_{i} \wedge Y_{i}<u_{i}$, and we use $r_{i j}=2^{(*)}$ if the conversion of $a_{i j}$ in 2 or $2^{*}$ does not change the equilibrium outcome.

1. $D_{1}=D_{2}=D_{3} \longrightarrow R$ shrinks to a $(3 \times 1)$ matrix and we have

$$
\left(\begin{array}{lll}
3 & 3 & 3
\end{array}\right) \longrightarrow D_{1}^{*}
$$

2. $D_{1} \neq D_{2}=D_{3} \longrightarrow R$ shrinks to a $(3 \times 2)$ matrix and we have

$$
\left(\begin{array}{lll}
3 & 3 & 2 \\
3 & 2 & 3
\end{array}\right) \longrightarrow\left\{\begin{array}{l}
D_{1}^{*} \\
D_{2}^{*}
\end{array} \quad\left(\begin{array}{lll}
3 & 3 & 2 \\
2 & 2 & 3
\end{array}\right) \longrightarrow D_{1}^{*}\right.
$$

3. $D_{1} \neq D_{2} \neq D_{3}$

(a) $X_{1}=Y_{1}=Z_{1} \wedge X_{2} \neq Y_{2} \neq Z_{2} \wedge X_{3} \neq Y_{3} \neq Z_{3}$

$$
\left(\begin{array}{lll}
3 & 3 & 1 \\
3 & 2^{(*)} & 2^{(*)} \\
3 & 1 & 3
\end{array}\right) \longrightarrow\left\{\begin{array}{l}
D_{1}^{*} \\
D_{3}^{*}
\end{array}\right.
$$

(b) $X_{1}=Y_{1} \neq Z_{1} \wedge X_{2}=Y_{2} \neq Z_{2} \wedge X_{3}=Y_{3} \neq Z_{3}$

$$
\left(\begin{array}{lll}
3 & 1 & 3 \\
3 & 3 & 1 \\
1 & 3 & 3
\end{array}\right) \longrightarrow\left\{\begin{array}{c}
D_{1}^{*} \\
D_{2}^{*} \\
D_{3}^{*}
\end{array}\right.
$$

(c) $X_{1}=Y_{1} \neq Z_{1} \wedge X_{2}=Y_{2} \neq Z_{2} \wedge X_{3} \neq Y_{3} \neq Z_{3}$

$$
\left(\begin{array}{lll}
3 & 1 & 3 \\
3 & 3 & 1 \\
1 & 3 & 2^{(*)}
\end{array}\right) \longrightarrow\left\{\begin{array}{l}
D_{1}^{*} \\
D_{2}^{*}
\end{array}\right.
$$


(d) $X_{1}=Y_{1} \neq Z_{1} \wedge X_{2} \neq Y_{2} \neq Z_{2} \wedge X_{3} \neq Y_{3} \neq Z_{3}$

$$
\begin{aligned}
& \left(\begin{array}{lll}
3 & 1 & 3 \\
3 & 3 & 1 \\
1 & 2^{(*)} & 2^{(*)}
\end{array}\right) \longrightarrow\left\{\begin{array}{l}
D_{1}^{*} \\
D_{2}^{*}
\end{array} \quad\left(\begin{array}{lll}
3 & 1 & 2^{(*)} \\
3 & 3 & 1 \\
1 & 2^{(*)} & 3
\end{array}\right) \longrightarrow D_{2}^{*}\right. \\
& \left(\begin{array}{lll}
3 & 1 & 2^{(*)} \\
3 & 2^{(*)} & 1 \\
1 & 3 & 3
\end{array}\right) \longrightarrow D_{3}^{*}
\end{aligned}
$$

(e) $X_{1} \neq Y_{1} \neq Z_{1} \wedge X_{2} \neq Y_{2} \neq Z_{2} \wedge X_{3} \neq Y_{3} \neq Z_{3}$

i. Doublemax: $=\left(\exists r_{i j}=r_{i k}=3 \quad j \neq k \quad i, j, k \in\{1,2,3\}\right)$

$$
\left(\begin{array}{lll}
3 & 3 & 2 \\
1 & 2^{(*)} & 3 \\
2^{(*)} & 2 & 1
\end{array}\right) \longrightarrow D_{1}^{*}
$$

ii. $Y_{1}<u_{1} \wedge Y_{2}<u_{2} \wedge Y_{3}<u_{3} \wedge$ no Doublemax

$$
\left(\begin{array}{lll}
1 & 3 & 2 \\
2 & 1 & 3 \\
3 & 2 & 1
\end{array}\right) \longrightarrow L^{*}
$$

iii. $Y_{1} \geq u_{1} \wedge Y_{2}<u_{2} \wedge Y_{3}<u_{3} \wedge$ no Doublemax

$$
\left(\begin{array}{lll}
1 & 3 & 2 \\
2^{*} & 1 & 3 \\
3 & 2 & 1
\end{array}\right) \longrightarrow D_{2}^{*}
$$

iv. $Y_{1} \geq u_{1} \wedge Y_{2} \geq u_{2} \wedge Y_{3}<u_{3} \wedge$ no Doublemax

$$
\begin{aligned}
& \left(\begin{array}{lll}
1 & 3 & 2 \\
2^{*} & 1 & 3 \\
3 & 2^{*} & 1
\end{array}\right) \longrightarrow\left\{\begin{array}{l}
D_{2}^{*} \\
D_{3}^{*}
\end{array} \quad\left(\begin{array}{lll}
1 & 1 & 3 \\
2^{*} & 3 & 2 \\
3 & 2^{*} & 1
\end{array}\right) \longrightarrow C_{23}^{*}\right. \\
& \left(\begin{array}{lll}
1 & 3 & 1 \\
3 & 1 & 2 \\
2^{*} & 2^{*} & 3
\end{array}\right) \longrightarrow D_{3}^{*}
\end{aligned}
$$

v. $Y_{1} \geq u_{1} \wedge Y_{2} \geq u_{2} \wedge Y_{3} \geq u_{3} \wedge$ no Doublemax

$$
\left(\begin{array}{lll}
1 & 3 & 2^{*} \\
2^{*} & 1 & 3 \\
3 & 2^{*} & 1
\end{array}\right) \longrightarrow\left\{\begin{array}{l}
D_{1}^{*} \\
D_{2}^{*} \\
D_{3}^{*}
\end{array} \quad\left(\begin{array}{lll}
1 & 1 & 3 \\
2^{*} & 3 & 2^{*} \\
3 & 2^{*} & 1
\end{array}\right) \longrightarrow C_{23}^{*}\right.
$$




\section{Division of the Cake}

In this section, we derive the overall equilibrium of the game. For that purpose, we have to compare the expected payoffs for the different players. Accordingly, we denote the expected payoff of Player $i$ by $\pi_{i}(i=1,2,3)$.

\subsection{Overall Equilibrium}

The solution of the game is given by the following theorem. ${ }^{8}$

\section{Theorem 1}

There exist two overall equilibria of the game

$$
\begin{aligned}
& D_{1}=(0,1,0) \\
& D_{2}=(1,0,0) \\
& D_{3}=\left(\frac{1}{2}, 0, \frac{1}{2}\right)
\end{aligned} \quad \text { or } \quad \begin{array}{ll}
D_{1}=(0,1,0) \\
D_{2}=(1,0,0) \\
D_{3}=\left(0, \frac{1}{2}, \frac{1}{2}\right)
\end{array}
$$

where $D_{3}=\left(\frac{1}{2}, 0, \frac{1}{2}\right)$ is a single-proposal equilibrium supported by Players 1 and 3, and $D_{3}=\left(0, \frac{1}{2}, \frac{1}{2}\right)$ is a single-proposal equilibrium supported by Players 2 and 3. As Player 3 is indifferent between $D_{3}=\left(\frac{1}{2}, 0, \frac{1}{2}\right)$ and $D_{3}=\left(0, \frac{1}{2}, \frac{1}{2}\right)$, he will make either proposal with a probability of $\frac{1}{2}$ (Tie-Break Rule 3 ). For the payoffs, this implies

$$
\pi_{1}=\frac{1}{4} \quad \pi_{2}=\frac{1}{4} \quad \pi_{3}=\frac{1}{2}
$$

The proof of the theorem follows directly from Corollary 1 and Propositions 5 and 6 in subsections 5.3, 5.4, and 5.5.

This theorem can be motivated as follows: If Player 1 makes any proposal $D_{1} \neq(0,1,0)$, Player 2 will counter it with a proposal $D_{2}$ such that $\pi_{1}$ is less than $\frac{1}{4}$. But by proposing $D_{1}=(0,1,0)$, Player 1 makes Player 2 so unattractive that any counter-proposal $D_{2} \neq(1,0,0)$ will give Player 2 a payoff $\pi_{2}$ of less than $\frac{1}{4}$. This implies that ultimately, the cake is divided half-by-half between Players 1 and 3 or Players 2 and 3 .

\subsection{Strategy of the Proof}

We prove Theorem 1 by backward induction. This is illustrated in the following steps:

Step 1: We determine the best response of Player 3 given any $D_{1}$ and $D_{2}$ and calculate the minimum payoff $\pi_{3}$, given specific relations between $D_{1}$ and $D_{2}$.

\footnotetext{
${ }^{8}$ The solution has been conjectured in Gersbach and Wehrspohn (2001).
} 
Step 2: Given $D_{1} \neq(0,1,0)$, we determine a proposal $D_{2}$ giving Player 2 a payoff of $\pi_{2}$ such that, together with the minimum payoff of $\pi_{3}$ of Player 3 from step 1 , the resource constraint implies $\pi_{1}<\frac{1}{4}$ for Player 1 .

Step 3: Given $D_{1}=(0,1,0)$, we calculate the payoff $\pi_{2}$ if Player 2 makes any proposal $D_{2} \neq(1,0,0)$ and compare this with his payoff $\pi_{2}$ when proposing $D_{2}=(1,0,0)$.

\subsection{Strategy of Player 3}

Given $D_{1}, D_{2}$, Player 3's ambition is to maximize his share of the cake by making an appropriate proposal $D_{3}$.

Given $D_{1}$ and $D_{2}$, Player 3 will generally consider four possible equilibrium outcomes when designing his proposal $D_{3}$.

I: $D_{3}$ is a single-proposal equilibrium.

II: $D_{1}$ or $D_{2}$ is a single-proposal equilibrium.

III: A correlated equilibrium arises.

IV: The cake is divided by drawing lots.

We now give examples of cases where the different designing principles (I-IV) are the best reactions of Player 3. The detailed rationalization of the best reaction of Player 3 will be given in the proof.

$$
\begin{aligned}
& \text { I } \left.\quad: \quad \begin{array}{l}
D_{1}=(0.7,0.3,0.0) \\
D_{2}=(0.3,0.5,0.2)
\end{array}\right\} \Longrightarrow D_{3}=(0.0,0.4,0.6) \quad \Longrightarrow D_{3}^{*} \\
& \left.\mathrm{II}^{9} \quad: \quad \begin{array}{l}
D_{1}=(0.1,0.3,0.6) \\
D_{2}=(0.5,0.5,0.0)
\end{array}\right\} \Longrightarrow D_{3}=(1.0,0.0,0.0) \quad \Longrightarrow D_{1}^{*} \\
& \text { III : } \left.\begin{array}{l}
D_{1}=(0.0,0.9,0.1) \\
D_{2}=(0.4,0.1,0.5)
\end{array}\right\} \Longrightarrow D_{3}=(0.2,0.0,0.8) \quad \Longrightarrow C_{23}^{*} \\
& \text { IV : } \left.\begin{array}{l}
D_{1}=(0.1,0.1,0.8) \\
D_{2}=(0.6,0.4,0.0)
\end{array}\right\} \Longrightarrow D_{3}=(0.0,0.7+\epsilon, 0.3-\epsilon) \Longrightarrow L^{*}
\end{aligned}
$$

with $\epsilon>0$ and infinitesimally small.

\footnotetext{
${ }^{9}$ In this case, we have a continuum of best proposals $D_{3}=\left(a_{31}, a_{32}, 1-a_{31}-a_{32}\right)$ with $a_{31} \in(0.9,1]$ and $a_{32} \in\left[0,1-a_{31}\right]$.
} 
The kind of equilibrium outcome (I-IV) Player 3 prefers depends strongly on the relations between the proposals $D_{1}$ and $D_{2}$, characterized by the submatrix $a$ of the proposal matrix $A$, which is defined by

$$
A=\left(\begin{array}{ccc}
a & a_{13} \\
a_{31} & a_{32} & a_{33}
\end{array}\right) \Longrightarrow a=\left(\begin{array}{ll}
a_{11} & a_{12} \\
a_{21} & a_{22}
\end{array}\right)
$$

and the corresponding rank matrix $\rho$, which is calculated from $a$ in a similar way as $R$ from $A$ :

$$
\rho=\phi[a]=\left(\begin{array}{ll}
\rho_{11} & \rho_{12} \\
\rho_{21} & \rho_{22}
\end{array}\right)
$$

where $\phi$ is defined in a similar way to $\Phi($ see (6)) as

$$
\phi: \rho_{i j}=\sum_{k=1}^{2} \Theta\left(a_{i j}-a_{k j}\right)
$$

The relevance of $a$ and $\rho$ will be shown in the proofs of the following propositions and corollaries.

\section{Proposition 1}

Given $D_{1}$ and $D_{2}$ and that $\rho$ is symmetric $\Longrightarrow$ determining the best reaction of Player 3 implies $\pi_{3} \geq \frac{3}{8}$.

\section{Corollary 1}

Given $D_{1}=(0,1,0)$ and $D_{2}=(1,0,0) \Longrightarrow$ Player 3's best proposal is $D_{3}=\left(0, \frac{1}{2}, \frac{1}{2}\right)$ or $D_{3}=\left(\frac{1}{2}, 0, \frac{1}{2}\right)$, which implies $\pi_{1}=\pi_{2}=\frac{1}{4}$ and $\pi_{3}=\frac{1}{2}$.

\section{Proposition 2}

Given $D_{1}, D_{2}, a \neq\left(\begin{array}{cc}0 & 0 \\ \frac{1}{2} & \frac{1}{2}\end{array}\right) \vee\left(\begin{array}{cc}\frac{1}{2} & \frac{1}{2} \\ 0 & 0\end{array}\right)$ and that $\rho$ is non-symmetric $\Longrightarrow$ determining the best reaction of Player 3 implies $\pi_{3}>\frac{1}{4}$.

\section{Corollary 2}

Given $a=\left(\begin{array}{cc}0 & 0 \\ \frac{1}{2} & \frac{1}{2}\end{array}\right) \vee\left(\begin{array}{cc}\frac{1}{2} & \frac{1}{2} \\ 0 & 0\end{array}\right) \Longrightarrow$ Player 3's best proposal is $D_{3}=\left(\frac{1}{2}+\epsilon, \frac{1}{4}, \frac{1}{4}-\epsilon\right)$ or $D_{3}=\left(\frac{1}{4}, \frac{1}{2}+\epsilon, \frac{1}{4}-\epsilon\right)$, which implies $\pi_{1}=\pi_{2}=\frac{7}{16}+\frac{\epsilon}{4}$ and $\pi_{3}=\frac{1}{8}-\frac{\epsilon}{2}$.

(The proofs are given in the appendix). 


\subsection{Strategy of Player 2}

In this section, we analyze possible reactions by Player 2 , given $D_{1}$ and the reaction of Player 3 derived in section 5.3.

For that purpose, we divide the proposal set of $D_{1}$ given by

$$
\mathcal{P}=\left\{\left(a_{11}, a_{12}\right) \mid\left(a_{11}, a_{12}\right) \in[0,1] \times\left[0,1-a_{11}\right]\right\}
$$

into four subsets:

$$
\begin{aligned}
\mathcal{A} & =\left[\frac{1}{2}, 1\right] \times\left[0,1-a_{11}\right] \backslash\left(\frac{1}{2}, \frac{1}{2}\right) \\
\mathcal{B} & =\left[0, \frac{1}{2}\right] \times\left[0,1-a_{11}\right] \backslash(0,1) \\
\mathcal{C} & =\left[0, \frac{1}{2}\right) \times\left[0, \frac{1}{2}\right) \\
D^{*} & =(0,1)
\end{aligned}
$$

\section{Proposition 3}

$\forall D_{1} \in \mathcal{A} \cup \mathcal{B} \exists$ a proposal ${ }^{10} D_{2}^{s}$ of Player 2 , such that $\rho$ is symmetric and $\pi_{2}>\frac{3}{8}$.

\section{Corollary 3}

If for $D_{1} \in \mathcal{A} \cup \mathcal{B} \exists$ a proposal ${ }^{11} D_{2}^{n s}$ of Player 2, such that $\rho$ is non-symmetric and $\pi_{2}\left(D_{2}^{n s}\right) \geq \pi_{2}\left(D_{2}^{s}\right) \Longrightarrow \pi_{2}\left(D_{2}^{n s}\right) \geq \frac{1}{2}$ or $\pi_{1}\left(D_{2}^{n s}\right)<\frac{1}{4}$.

\section{Proposition 4}

$\forall D_{1} \in \mathcal{C} \exists$ a proposal $D_{2}^{n s}$ of Player 2 , such that $\rho$ is non-symmetric and $\pi_{2} \geq \frac{1}{2}$ or the best proposal $D_{2}^{n s}$ with $\rho$ non-symmetric for Player 2 implies $\pi_{1}=0$.

\section{Corollary 4}

If for $D_{1} \in \mathcal{C} \exists$ a proposal $D_{2}^{s}$ of Player 2 , such that $\rho$ is symmetric and $\pi_{2}\left(D_{2}^{s}\right) \geq$ $\pi_{2}\left(D_{2}^{n s}\right) \Longrightarrow \pi_{2}\left(D_{2}^{s}\right)>\frac{3}{8}$.

\section{Proposition 5}

Given $D_{1}=(0,1,0)$, the best reaction of Player 2 is $D_{2}=(1,0,0)$.

(The proofs are given in the appendix).

\footnotetext{
${ }^{10} D_{2}^{s}$ is a proposal made by Player 2 such that $a$ is symmetric.

${ }^{11} D_{2}^{n s}$ is a proposal made by Player 2 such that $a$ is non-symmetric.
} 


\subsection{Strategy of Player 1}

Since Player 1 anticipates the reactions of Players 2 and 3, we obtain the following proposition:

\section{Proposition 6}

The best proposal of Player 1 is $D_{1}=(0,1,0)$.

\section{Proof of Proposition 6}

(i) Suppose $D_{1} \neq(0,1,0)$ and $D_{2}$ with $\rho$ symmetric is the best reaction of Player 2 $\Longrightarrow \pi_{3} \geq \frac{3}{8}$ (Proposition 1) and $\pi_{2}>\frac{3}{8}$ (Proposition 3 and Corollary 3 ) $\Longrightarrow$ together with the resource constraint, we obtain $\pi_{1}+\stackrel{>\frac{3}{8}}{\pi_{2}^{8}}+\stackrel{2}{\pi_{3}^{8}}=1 \Longrightarrow \pi_{1}<\frac{1}{4}$.

(ii) Suppose $D_{1} \neq(0,1,0)$ and $D_{2}$ with $\rho$ non-symmetric is the best reaction of Player 2

$\Longrightarrow \pi_{3}>\frac{1}{4}$ Proposition $^{12} 2$ ) and $\pi_{2} \geq \frac{1}{2} \vee \pi_{1}<\frac{1}{4}$ (Corollary 3 and Proposition 4) $\Longrightarrow$ together with the resource constraint, we obtain $\pi_{1}+\stackrel{2 \frac{1}{2}}{\pi_{2}}+\stackrel{>^{\frac{1}{4}}}{\pi_{3}}=1 \Longrightarrow \pi_{1}<\frac{1}{4}$, or we have directly $\pi_{1}<\frac{1}{4}$.

$\Longrightarrow$ (i) and (ii), together with Corollary 1 and Proposition 5, imply that $D_{1}=(0,1,0)$ is the best proposal for Player 1 .

\section{Discussion and Conclusion}

We have examined a common collective choice process to study the allocation of resources among a group of people. The analysis reveals that the first two agents want to make each other as unattractive as possible with regard to the third agent's proposal. To do so, they offer each other the whole cake, and the third player can ensure that he obtains one half of the cake.

This outcome exhibits a powerful last-mover advantage, whereas the other players are forced to make strategic proposals involving zero resources for themselves. They expect $\frac{1}{4}$ of the cake. Additionally, it seems surprising that the first player can totally

\footnotetext{
${ }^{12}$ Note that neither $D_{2}=\left(\frac{1}{2}, \frac{1}{2}, 0\right)$ is the best reaction of Player 2 given that $D_{1}=(0,0,1)$, nor is $D_{2}=(0,0,1)$ the best reaction of Player 2 given that $D_{1}=\left(\frac{1}{2}, \frac{1}{2}, 0\right)$.
} 
outweigh the second-mover advantage of the second player. The way in which these characteristics extend to group decisions with a larger number of individuals is an important avenue to future research. Of course, there is a variety of game-theoretic considerations and alternative refinement concepts that can be examined. How robust our main findings are with regard to such extensions remains to be explored. A further useful extension of our framework would be to consider the role of risk preferences. A large literature has generated the finding that it is disadvantageous to be relatively risk averse in bargaining settings. ${ }^{13}$ Harrington (1989 and 1990) has, however, shown that in bargaining games in which acceptance of a proposed allocation only requires the approval of a majority, it is advantageous for a player to be relatively risk averse. How risk aversion in our model affects the utility of each member would be be an interesting research project.

\footnotetext{
${ }^{13}$ At least since Zeuthen (1930), risk preferences have been thought to be an important determinant of the outcome of bargaining. The role of risk preferences in a bargaining setting has been examined using both an axiomatic framework [see Roth (1979), Kihlstrom, Roth and Schmeidler (1981), Nielsen (1984)] and a non-cooperative game framework [Roth (1985), Binmore, Rubinstein and Wolinksy (1986) and Harrington (1986)]. These studies have confirmed that the more risk averse an agent is, the lower his share. Notable exceptions to this finding are described by Roth and Rothblum (1982) and Osborne (1985).
} 


\section{Appendix}

We have already mentioned that the specific relations between $D_{1}$ and $D_{2}$ are relevant for the construction of the best reaction $D_{3}$ given by $\rho$ and $a$. Now we need a more detailed specification of the relations between the entries of $a$. Therefore we define

$$
\begin{aligned}
\mu_{j} & =\frac{1}{2}\left(a_{1 j}+a_{2 j}\right) \\
\bar{\mu} & =\max \left\{\mu_{1}, \mu_{2}\right\} \\
\underline{\mu} & =\min \left\{\mu_{1}, \mu_{2}\right\} \\
\bar{x}_{j} & =\max \left\{a_{1 j}, a_{2 j}\right\} \\
y_{j} & =\min \left\{a_{1 j}, a_{2 j}\right\} \\
\underline{x} & =\min \left\{x_{1}, x_{2}\right\}
\end{aligned}
$$

\subsection{Proof of Propositions 1 and 2 and Corollaries 1 and 2}

Before we start, note that the best reaction $D_{3}$ is not always unique (i.e. $x_{1}=x_{2} \wedge y_{1}=$ $y_{2}$, or see footnote 9). But as we only want to determine the minimum share of the cake for Player 3, it is sufficient to give only one best reaction.

\subsubsection{Proof of Proposition 1}

There are three different possibilities for $\rho$ to be symmetric:

$$
\rho_{1}=\left(\begin{array}{ll}
2 & 1 \\
1 & 2
\end{array}\right) \quad \rho_{1^{\prime}}=\left(\begin{array}{ll}
1 & 2 \\
2 & 1
\end{array}\right) \quad \rho_{2}=\left(\begin{array}{ll}
2 & 2 \\
2 & 2
\end{array}\right)
$$

For $\rho_{2}$ we have $D_{1}=D_{2}$, and the best proposal $D_{3}$ is given by

1. If $D_{1}=(0,0,1) \Rightarrow D_{3}=(0,0,1)$

2. If $D_{1} \neq(0,0,1) \Rightarrow D_{3}=(\underline{x}+\epsilon, 0,1-\underline{x}-\epsilon)$ if $x_{1}=\underline{x}$ and $D_{3}=(0, \underline{x}+\epsilon, 1-\underline{x}-\epsilon)$ if $x_{2}=\underline{x}$.

For $\rho_{1}$ or $\rho_{1^{\prime}}$ it is sufficient to analyze $\rho_{1}$ only, as the same arguments follow for $\rho_{1^{\prime}}$ by exchanging the columns in matrix $a$.

For $\rho_{1} A$ is given by

$$
A=\left(\begin{array}{ccc}
x_{1} & y_{2} & a_{13} \\
y_{1} & x_{2} & a_{23} \\
a_{31} & a_{32} & a_{33}
\end{array}\right) \quad \text { with } \quad \begin{aligned}
& a_{13}=1-x_{1}-y_{2} \\
& a_{23}=1-x_{2}-y_{1} \\
& \\
& a_{33}=1-a_{31}-a_{32}
\end{aligned}
$$


To calculate the best reaction of Player 3, the following properties of $A$ are relevant:

(i) $x_{i}>a_{3 i} \geq \mu_{i} \Longrightarrow \Phi\left(x_{i}\right)=3, \Phi\left(y_{i}\right)=1, \Phi\left(a_{3 i}\right)=2^{*}$

(ii) $x_{i}>y_{i}>a_{3 i}>2 y_{i}-x_{i} \Longrightarrow \Phi\left(x_{i}\right)=3, \Phi\left(y_{i}\right)=2, \Phi\left(a_{3 i}\right)=1$

(iii) $x_{i}>y_{i}>a_{3 i}$ and $a_{3 i} \leq 2 y_{i}-x_{i} \Longrightarrow \Phi\left(x_{i}\right)=3, \Phi\left(y_{i}\right)=2^{*}, \Phi\left(a_{3 i}\right)=1$

(iv) $x_{i}=a_{3 i}>y_{i} \Longrightarrow \Phi\left(x_{i}\right)=3, \Phi\left(y_{i}\right)=1, \Phi\left(a_{3 i}\right)=3$

With these properties we distinguish six different kinds of proposals $D_{3}$ discussed in detail below:

$$
D_{3}^{\underline{\mu}} \quad D_{3}^{\bar{\mu}} \quad D_{3}^{\mu_{i}} \quad D_{3}^{\underline{x}} \quad D_{3}^{C o r} \quad D_{3}^{L}
$$

$D_{3} \frac{\mu}{3}$ : Player 3 offers Player 1 or $2 \mu$ and keeps the rest for himself, such that $D_{3}$ is a unique equilibrium and he receives a payoff $\pi_{3}=1-\underline{\mu}$. For example

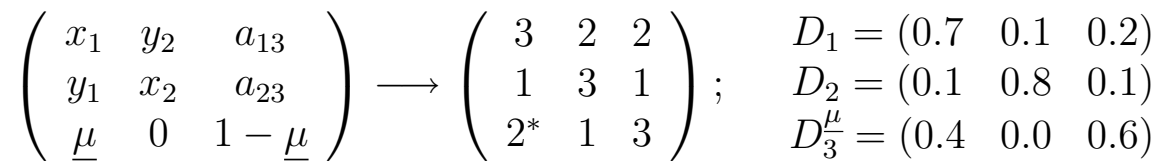

$D_{3}^{\bar{\mu}}$ : Player 3 offers Player 1 or $2 \bar{\mu}$ and keeps the rest for himself, such that $D_{3}$ is a unique equilibrium and he receives a payoff $\pi_{3}=1-\bar{\mu}$. For example

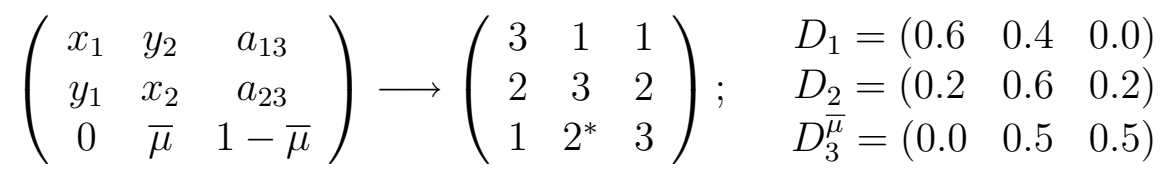

$D_{3}^{\mu_{i}}$ : Player 3 offers Player $i a_{3 i}=\mu_{i}$, Player $j a_{3 j}=2 y_{j}-x_{j}+\epsilon$ and keeps the rest for himself, such that $D_{3}$ is a unique equilibrium and he receives a payoff $\pi_{3}=1-\left(\mu_{i}+2 y_{j}-x_{j}+\epsilon\right)\left(i, j=1,2 i \neq j\right.$ and $\left.2 y_{j}-x_{j} \geq 0\right)$. For example

$$
\begin{aligned}
& \left(\begin{array}{ccc}
x_{1} & y_{2} & a_{13} \\
y_{1} & x_{2} & a_{23} \\
\mu_{1} & 2 y_{2}-x_{2}+\epsilon & 1-\left(\mu_{1}+2 y_{2}-x_{2}+\epsilon\right)
\end{array}\right) \longrightarrow\left(\begin{array}{ccc}
3 & 2 & 2 \\
1 & 3 & 1 \\
2^{*} & 1 & 3
\end{array}\right) \\
& D_{1}=\left(\begin{array}{lll}
0.58 & 0.38 & 0.04
\end{array}\right. \\
& D_{2}=\left(\begin{array}{lll}
0.32 & 0.68 & 0.00
\end{array}\right) \\
& D_{3}^{\mu_{1}}=\left(\begin{array}{lll}
0.45 & 0.08+\epsilon & 0.47-\epsilon
\end{array}\right)
\end{aligned}
$$

$D^{\underline{x}}$ : Player 3 offers Player 1 or $2 \underline{x}$ and keeps the rest for himself, such that $D_{3}$ is a unique equilibrium and he receives a payoff $\pi_{3}=1-\underline{x}$. For example

$$
\left(\begin{array}{ccc}
x_{1} & y_{2} & a_{13} \\
y_{1} & x_{2} & a_{23} \\
\underline{x} & 0 & 1-\underline{x}
\end{array}\right) \longrightarrow\left(\begin{array}{ccc}
3 & 2^{*} & 1 \\
2 & 3 & 2 \\
3 & 1 & 3
\end{array}\right) ; \quad \begin{aligned}
& D_{1}=\left(\begin{array}{lll}
0.52 & 0.40 & 0.08
\end{array}\right) \\
& D_{2}=\left(\begin{array}{lll}
0.30 & 0.54 & 0.16
\end{array}\right) \\
& D_{3}^{\underline{x}}=\left(\begin{array}{lll}
0.52 & 0.00 & 0.48
\end{array}\right)
\end{aligned}
$$


$D_{3}^{C o r}$ : Player 3 offers Player $i a_{3 i}=\mu_{i}$, Player $j a_{3 j}=0$

and keeps the rest for himself, such that $D_{3}$ is part of the correlated equilibrium and he receives a payoff $\pi_{3}=\frac{1}{2}\left(1-\mu_{i}+a_{i 3}\right)$. For example

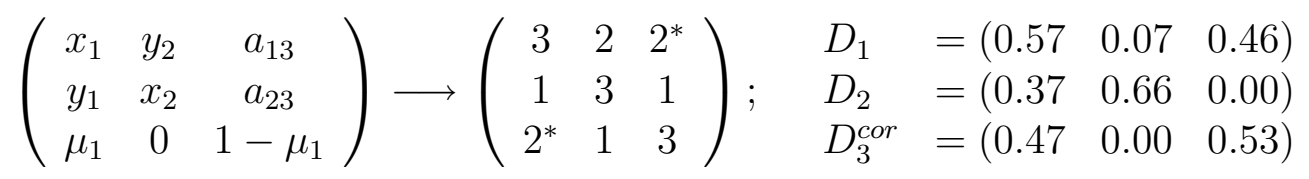

$D_{3}^{T}$ : (a) Player 3 proposes $D_{3}=(0,0,1)$ if $2 y_{i}<x_{i}(i=1,2)$, such that the proposal is selected by drawing lots and he receives a payoff $\pi_{3}=1-\frac{1}{3}\left(x_{1}+x_{2}+y_{1}+y_{2}\right)$. For example

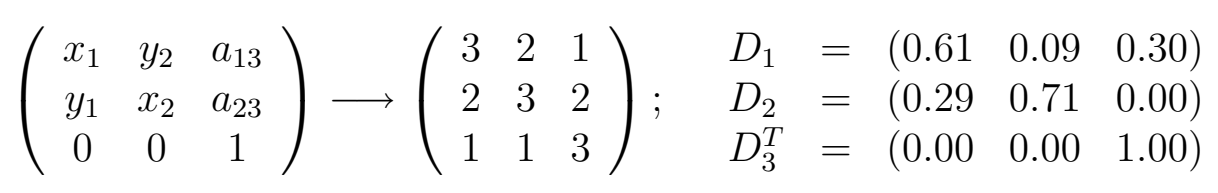

(b) Player 3 offers Player $i a_{3 i}=2 y_{i}-x_{i}+\epsilon$ if $2 y_{i} \geq x_{i}$ and Player $j a_{3 j}=0$ if $2 y_{j}<x_{j}(i \neq j)$ and keeps the rest for himself, such that the proposal is selected by drawing lots and he receives a payoff $\pi_{3}=1-\frac{1}{3}\left(3 y_{i}+x_{j}+y_{j}+\epsilon\right)$. For example

$$
\begin{aligned}
& \left(\begin{array}{ccc}
x_{1} & y_{2} & a_{13} \\
y_{1} & x_{2} & a_{23} \\
0 & 2 y_{2}-x_{2}+\epsilon_{2} & 1-\left(2 y_{2}-x_{2}+\epsilon_{2}\right)
\end{array}\right) \longrightarrow\left(\begin{array}{ccc}
3 & 2 & 1 \\
2 & 3 & 2 \\
1 & 1 & 3
\end{array}\right) \\
& \begin{array}{l}
D_{1}=\left(\begin{array}{lll}
0.58 & 0.30 & 0.12
\end{array}\right) \\
D_{2}=\left(\begin{array}{lll}
0.05 & 0.55 & 0.40
\end{array}\right) \\
D_{3}^{T}=\left(\begin{array}{lll}
0.00 & 0.05+\epsilon & 0.95-\epsilon
\end{array}\right)
\end{array}
\end{aligned}
$$

Note that constructing $D_{3}$ in such a way that $D_{1}$ or $D_{2}$ are unique equilibria or $D_{1}$ and $D_{2}$ form a correlated equilibrium can never be better than $D_{3}^{\underline{x}}$, since $1-\underline{x} \geq a_{i 3}(i=$ $1,2)$ and offering $D_{3}^{\underline{x}}$ is always possible for Player 3 if $\rho$ symmetric. This ensures that Refinement 4 can always be satisfied and Player 3's best reaction is an element of $\left\{D_{3}^{\frac{\mu}{3}}, D_{3}^{\bar{\mu}}, D_{3}^{\mu_{i}}, D_{3}^{\frac{x}{3}}, D_{3}^{C o r}, D_{3}^{T}\right\}$

In the following we calculate for every proposal $D_{3}^{\underline{\mu}}, D_{3}^{\bar{\mu}}, D_{3}^{\mu_{i}}, D_{3}^{\underline{x}}, D_{3}^{C o r}$ and $D_{3}^{L}$ the feasible set in which it is the best reaction of Player 3. Also, we minimize the payoff $\pi_{3}$ giving the best reaction and the feasible set. In the course of these calculations we will often draw upon the argument of contradiction. Accordingly, we introduce the sign 4 to indicate a conclusion that contradicts the assumptions. Since every feasible set is bounded by linear inequalities we can calculate $\min \left\{\pi_{3}\right\}$ given the best reaction of Player 3 by a simplex algorithm. ${ }^{14}$

\footnotetext{
${ }^{14}$ This minimization is done by Maple. Since the constraints are linear, there exists a finite algorithm for calculating the extrema. These are located in the corners of the 4-dimensional hyper-polyeder. The number of constraints does not exceed 30 . Thus the problem has less than $\left(\begin{array}{c}30 \\ 4\end{array}\right) \approx 10^{5}$ corners, which can be checked in a few seconds using a $2 \mathrm{GHz}$ processor.
} 
1. $D^{\underline{\mu}}$

Constraints on $D^{\frac{\mu}{3}}$ being the best proposal for Player 3 (w.l.o.g $\mu_{1}=\underline{\mu}$ ):

$\left.\begin{array}{|r|rr|}\hline 1 . & \mu_{1} \leq \mu_{2} \\ \hline 2 . & 2 y_{2} & <x_{2} \\ \hline 3 . & a_{i 3} & \leq a_{j 3} \\ \hline 4 . & 1-\mu_{1}+a_{i 3} & >2 a_{j 3}\end{array}\right\} i, j=1,2 i \neq j$

- Drawing lots versus $D_{\frac{\mu}{3}}$ :

$$
\begin{aligned}
& u_{3}^{\max }=\frac{1}{31_{\leq} \mu_{2}}=\overbrace{1-x_{1}-y_{2}}^{a_{13}}+\overbrace{1-x_{2}-y_{1}}^{a_{23}}+\overbrace{1}^{a_{33}^{\text {max }}}) \\
& \stackrel{\mu 1_{\leq} \mu_{2}}{\leq} 1-\frac{2}{3}\left(x_{1}+y_{1}\right)<1-\frac{1}{2}\left(x_{1}+y_{1}\right)=1-\underline{\mu}=a_{33}\left(D_{3}^{\underline{\mu}}\right)
\end{aligned}
$$

- Correlated equilibrium versus $D_{3}^{\frac{\mu}{3}}$ :

$1-\underline{\mu}>\frac{1}{2}\left(1-\underline{\mu}+a_{i 3}\right), i=1,2$.

- Simplex minimization for Player 3:

$$
\pi_{3}^{\min }\left(D^{\frac{\mu}{3}}\right)=\frac{1}{2}
$$

$$
\begin{aligned}
& \text { General example: } \quad\left(\begin{array}{ccc}
0.6 & 0.3 & 0.1 \\
0.2 & 0.8 & 0.0 \\
0.4 & 0.0 & 0.6
\end{array}\right) \\
& \text { Minium payoff example: } \quad\left(\begin{array}{ccc}
\frac{7}{10} & \frac{3}{10} & 0 \\
\frac{3}{10} & \frac{7}{10} & 0 \\
\frac{1}{2} & 0 & \frac{1}{2}
\end{array}\right)
\end{aligned}
$$

2. $D_{3}^{\bar{\mu}}$

Constraints on $D_{3}^{\bar{\mu}}$ being the best proposal for Player 3 (w.l.o.g $\mu_{2}=\bar{\mu}$ ):

\begin{tabular}{|r|rc|}
\hline 1. & $2 y_{2}$ & $\geq x_{2}$ \\
\hline 2. & $2 y_{1}$ & $<x_{1}$ \\
\hline 3. & $\mu_{2}$ & $\leq \underline{x}$ \\
\hline 4. & $a_{i 3}$ & $\leq a_{j 3}$ \\
\hline 5. & $1-\mu_{2}+a_{i 3}$ & $>2 a_{j 3}$ \\
\hline 6. & $\mu_{1}+2 y_{2}-x_{2}$ & $\geq \mu_{2}$ \\
\hline
\end{tabular}

- Drawing lots versus $D_{3}^{\bar{\mu}}$ :

$$
\begin{aligned}
u_{3}^{\max } & =\frac{1}{3}(\overbrace{1-x_{1}-y_{2}}^{a_{13}}+\overbrace{1-x_{2}-y_{1}}^{a_{23}}+\overbrace{1-\left(2 y_{2}-x_{2}\right)}^{a_{33}^{\max }}) \\
& =1-\frac{1}{3}\left(x_{1}+y_{1}\right)-y_{2}
\end{aligned}
$$


We have

$$
\begin{aligned}
& x_{1}+y_{1} \stackrel{\mu_{2} \leq \underline{x}}{\geq} \frac{1}{2}\left(x_{2}+y_{2}\right) \stackrel{2 y_{2} \geq x_{2}}{\geq} \frac{3}{2}\left(x_{2}-y_{2}\right) \\
& \Longrightarrow \underbrace{1-\frac{1}{3}\left(x_{1}+y_{1}\right)-y_{2}}_{u_{3}^{\max }} \leq \underbrace{1-\frac{1}{2}\left(x_{2}+y_{2}\right)}_{a_{33}\left(D_{3}^{\bar{\mu}}\right)}
\end{aligned}
$$

- Correlated equilibrium versus $D_{3}^{\bar{\mu}}$ :

Suppose $\pi_{3}$ (Correlated equilibrium) $>a_{33}\left(D_{3}^{\bar{\mu}}\right) \Longrightarrow$

$$
\overbrace{\frac{1}{2}\left(1-\mu_{1}+1-x_{1}-y_{2}\right)}^{\pi_{3}^{\max }(\text { Correlated equilibrium) }}>\overbrace{1-\mu_{2}}^{a_{33}\left(D_{3}^{\bar{\mu}}\right)} \Longrightarrow \underbrace{\frac{1}{2}\left(y_{1}-x_{1}\right)}_{<0}>\underbrace{2-x_{2}}_{>0}
$$

- Simplex minimization for Player 3:

$$
\pi_{3}^{\min }\left(D_{3}^{\bar{\mu}}\right)=\frac{3}{7}
$$

$$
\begin{aligned}
& \text { General example: } \quad\left(\begin{array}{ccc}
0.6 & 0.34 & 0.06 \\
0.2 & 0.50 & 0.30 \\
0.0 & 0.42 & 0.58
\end{array}\right) \\
& \text { Minium payoff example: } \quad\left(\begin{array}{ccc}
\frac{4}{7}-\epsilon & \frac{3}{7}+\epsilon & 0 \\
\frac{2}{7}+\epsilon & \frac{5}{7}-\epsilon & 0 \\
0 & \frac{4}{7} & \frac{3}{7}
\end{array}\right)
\end{aligned}
$$

3. $D_{3}^{\mu_{i}}$

Constraints on $D_{3}^{\mu_{i}}$ being the best proposal for Player 3 (w.l.o.g. we consider only the constraints for $\left.D_{3}^{\mu_{1}}\right)$ :

(i)

$\left.\begin{array}{|r|rl|}\hline 1 . & 2 y_{2} & \geq x_{2} \\ \hline 2 . & 2 y_{1} & <x_{1} \\ \hline 3 . & \mu_{1}+2 y_{2}-x_{2} & <\underline{x} \\ \hline 4 . & \mu_{1}+2 y_{2}-x_{2} & <\mu_{2} \\ \hline 5 . & a_{i 3} & \leq a_{j 3} \\ \hline 6 . & 1-\left(\mu_{1}+2 y_{2}-x_{2}+\epsilon\right)+a_{i 3} & >2 a_{j 3}\end{array}\right\} i, j=1,2 i \neq j$

- Drawing lots versus $D_{3}^{\mu_{1}}$ :

(a) $\mu_{2}<\underline{x}$ see argumentation for $D_{3}^{\bar{\mu}}$.

(b) $\mu_{2} \geq \underline{x} \Longrightarrow \underline{x}=x_{1}$ and with $\mu_{1}+2 y_{2}-x_{2}<x_{1} \Longrightarrow$

$x_{1}+y_{1}<2 x_{1}+2 x_{2}-4 y_{2}<4\left(x_{2}-y_{2}\right)<6\left(x_{2}-y_{2}\right) \Longrightarrow$

$u_{3}^{\max }=1-\frac{1}{3}\left(x_{1}+y_{1}\right)-y_{2}<1-\mu_{1}-\left(2 y_{2}-x_{2}\right)=a_{33}\left(D_{3}^{\mu_{1}}\right)$ 
- Correlated equilibrium versus $D_{3}^{\mu_{i}}$

For constraint set (i) we have $\mu_{2}>\mu_{1}+2 y_{2}-x_{2}$

- Simplex minimization for Player 3:

$$
\pi_{3}^{\min }\left(D_{3}^{\mu_{i}}(i)\right)>\frac{3}{7}
$$

General example: $\quad\left(\begin{array}{ccc}0.55 & 0.4 & 0.15 \\ 0.27 & 0.7 & 0.03 \\ 0.41 & 0.1+\epsilon & 0.49-\epsilon\end{array}\right)$

Minium payoff example: $\quad\left(\begin{array}{ccc}\frac{4}{7} & \frac{3}{7} & 0 \\ \frac{2}{7}-2 \epsilon & \frac{7}{10} & 2 \epsilon \\ \frac{3}{7}-\epsilon & \frac{1}{7} & \frac{3}{7}+\epsilon\end{array}\right)$

(ii)

\begin{tabular}{|c|c|c|c|}
\hline 1. & $2 y_{1}$ & $\geq$ & $x_{1}$ \\
\hline 2. & $2 y_{2}$ & $\geq$ & $x_{2}$ \\
\hline 3. & $\mu_{1}+2 y_{2}-x_{2}$ & $<$ & $\underline{x}$ \\
\hline 4. & $\mu_{1}+2 y_{2}-x_{2}$ & $\leq$ & $\mu_{2}+2 y_{1}-x_{1}$ \\
\hline
\end{tabular}

- Drawing lots versus $D_{3}^{\mu_{1}}$ :

$$
\begin{aligned}
u_{3}^{\max } & =\frac{1}{3}(\overbrace{1-x_{1}-y_{2}}^{a_{13}}+\overbrace{1-x_{2}-y_{1}}^{a_{23}}+\overbrace{1-2 y_{1}+x_{1}-\epsilon-2 y_{2}+x_{2}-\epsilon^{\prime}}^{a_{33}^{\max }}) \\
& =1-\left(y_{1}+y_{2}\right)-\frac{1}{3}\left(\epsilon+\epsilon^{\prime}\right)
\end{aligned}
$$

and

$$
\begin{array}{cc}
y_{1}+y_{2} \stackrel{\substack{2 y_{1} \geq x_{1} \\
2 y_{2} \geq x_{2}}}{\geq} \underline{x} & \Longrightarrow \\
\underbrace{1-\left(y_{1}+y_{2}\right)-\frac{1}{3}\left(\epsilon+\epsilon^{6}\right)}_{u_{3}^{\max }}<1-\underline{x} \stackrel{\substack{\mu_{1}+2 y_{2}-x_{2}<\underline{x}}}{\leq} \underbrace{1-\left(\mu_{1}+2 y_{2}-x_{2}+\epsilon\right)}_{a_{33}\left(D_{3}^{\mu_{1}}\right)} \\
\left(\epsilon \leq \underline{x}-\left(\mu_{1}+2 y_{2}-x_{2}\right)\right.
\end{array}
$$

- Correlated equilibrium versus $D_{3}^{\mu_{i}}$

$\mu_{2}+2 y_{1}-x_{1} \geq \mu_{1}+2 y_{2}-x_{2}$

- Simplex minimization for Player 3:

$$
\pi_{3}^{\min }\left(D_{3}^{\mu_{i}}(i i)\right)>\frac{3}{8}
$$

- Examples 
General example: $\quad\left(\begin{array}{ccc}0.55 & 0.4 & 0.05 \\ 0.27 & 0.7 & 0.03 \\ 0.41 & 0.1+\epsilon & 0.49-\epsilon\end{array}\right)$

Minium payoff example: $\quad\left(\begin{array}{ccc}\frac{5}{8}+2 \epsilon & \frac{3}{8}-4 \epsilon & 2 \epsilon \\ \frac{3}{7} & \frac{5}{8} & 0 \\ \frac{4}{8}+\epsilon & \frac{1}{8}-8 \epsilon+\epsilon^{\prime} & \frac{3}{8}+7 \epsilon-\epsilon^{\prime}\end{array}\right) \quad\left(\epsilon^{\prime}<\epsilon\right)$

4. $D^{\frac{x}{3}}$

Constraints on $D_{\frac{x}{3}}^{x}$ being the best proposal for Player 3:

(i)

\begin{tabular}{|l|rc|}
\hline 1. & $2 y_{2}$ & $\geq x_{2}$ \\
\hline 2. & $2 y_{1}$ & $<x_{1}$ \\
\hline 3. & $\mu_{2}$ & $\geq \underline{x}$ \\
\hline 4. & $\mu_{1}+2 y_{2}-x_{2}$ & $>\underline{x}$ \\
\hline
\end{tabular}

- Drawing lots versus $D^{\underline{x}}$ :

$$
u_{3}^{\max }=\frac{1}{3}(a_{13}+a_{23}+\underbrace{1-\left(\mu_{1}+2 y_{2}-x_{2}+\epsilon\right)}_{a_{33}\left(D_{\frac{x}{3}}\right)}) \stackrel{\substack{a_{13} \leq 1-\underline{x} \\ a_{23} \leq 1-\underline{x}}}{\leq} a_{33}\left(D_{3}^{\frac{x}{3}}\right)
$$

- Correlated equilibrium versus $D_{3}^{\underline{x}}$ :

For a correlated equilibrium we need $\mu_{1}=\underline{\mu}$. Otherwise it could not be better than $D_{3}^{\underline{x}}$, since $1-\mu_{1}$ is part of the correlated payoff of Player 3 and $1-\mu_{1} \leq 1-\underline{x}$.

Additionally we need the following rank matrix:

$$
\left(\begin{array}{ccc}
x_{1} & y_{2} & 1-x_{1}-y_{2} \\
y_{1} & x_{2} & 1-y_{1}-x_{2} \\
\mu_{1} & 0 & 1-\mu_{1}
\end{array}\right) \longrightarrow\left(\begin{array}{ccc}
3 & 2^{*} & 2^{*} \\
1 & 3 & 1 \\
2^{*} & 1 & 3
\end{array}\right)
$$

This implies the following three conditions for the existence of a correlated equilibrium:

(a) $\mu_{1}<\mu_{2}$

(b) $a_{23}<a_{13}$

(c) $1-\mu_{1}+a_{23}<2 a_{13}$

Two cases are possible $x_{1}=\underline{x} \vee x_{2}=\underline{x}$

(a) Suppose $x_{1}=\underline{x} \Longrightarrow$

$$
\begin{aligned}
& 1-\mu_{1}+a_{23}<2 a_{13} \Longleftrightarrow 3 x_{1}+4 y_{2}<3 y_{1}+2 x_{2} \text { and } \\
& 2 y_{1}<x_{1} \Longleftrightarrow 3 x_{1}+4 y_{2}>6 y_{1}+4 y_{2} \stackrel{2 y_{2} \geq x_{2}}{\geq} 6 y_{1}+2 x_{2} \geq 3 y_{1}+2 x_{2}
\end{aligned}
$$


(b) Suppose $x_{2}=\underline{x} \Longrightarrow \rho$ is no longer symmetric.

- Simplex minimization for Player 3:

$$
\pi_{3}^{\min }\left(D \frac{x}{3}(i)\right)>\frac{3}{7}
$$

- Examples

$$
\begin{aligned}
& \text { General example: } \quad\left(\begin{array}{ccc}
0.55 & 0.45 & 0.00 \\
0.27 & 0.72 & 0.01 \\
0.55 & 0.00 & 0.45
\end{array}\right) \\
& \text { Minium payoff example: } \quad\left(\begin{array}{ccc}
\frac{4}{7}-\epsilon & \frac{3}{7}+\epsilon & 0 \\
\frac{2}{7}-11 \epsilon & \frac{5}{7}-3 \epsilon & 14 \epsilon \\
\frac{4}{7}-\epsilon & 0 & \frac{3}{7}+\epsilon
\end{array}\right)
\end{aligned}
$$

(ii)

\begin{tabular}{|l|rl|}
\hline 1. & $2 y_{2}$ & $\geq x_{2}$ \\
\hline 2. & $2 y_{1}$ & $<x_{1}$ \\
\hline 3. & $\mu_{2}$ & $\leq \underline{x}$ \\
\hline 4. & $\mu_{1}+2 y_{2}-x_{2}$ & $\geq \mu_{2}$ \\
\hline $5 .^{15}$ & $a_{13}$ & $\leq a_{23}$ \\
\hline 6. & $1-\mu_{2}+a_{13}$ & $\leq 2 a_{23}$ \\
\hline 7. & $\frac{1}{2}\left(1-\mu_{2}+a_{23}\right)$ & $\leq 1-\underline{x}$ \\
\hline
\end{tabular}

- Drawing lots versus $D_{3}^{\frac{x}{3}}$ :

If $\left(x_{1}=\underline{x}\right)$ constraint (4.) fails and if $\left(x_{2}=\underline{x}\right)$ the additionally required constraint $\frac{1}{3}\left(1-\left(2 y_{2}-x_{2}\right)+a_{13}+a_{23}\right)>1-\underline{x}$ fails.

- Correlated equilibrium versus $D_{3}^{\underline{x}}$ :

See constraint (7.)

- Simplex minimization for Player 3:

$$
\pi_{3}^{\min }\left(D_{3}^{x}(i i)\right)=\frac{3}{7}
$$

- Examples

$$
\begin{aligned}
& \text { General example: } \quad\left(\begin{array}{ccc}
0.55 & 0.45 & 0.00 \\
0.07 & 0.56 & 0.37 \\
0.55 & 0.00 & 0.45
\end{array}\right) \\
& \text { Minium payoff example: } \quad\left(\begin{array}{ccc}
\frac{4}{7} & \frac{3}{7} & 0 \\
\frac{1}{14} & \frac{4}{7} & \frac{5}{14} \\
\frac{4}{7} & 0 & \frac{3}{7}
\end{array}\right)
\end{aligned}
$$

\footnotetext{
${ }^{15}$ Note that $a_{13}>a_{23}$ is not possible. Suppose $a_{13}>a_{23} \Longrightarrow$ condition (6.) converts to $1-\mu_{2}+a_{23} \leq$ $2 a_{13} \Longrightarrow 4 x_{1}-2 y_{1}+3 y_{2} \leq 3 x_{2} \stackrel{\mu_{2} \leq \mu_{1}+2 y_{2}-x_{2}}{\leq} x_{1}+y_{1}+3 y_{2} \Longrightarrow 3 x_{1} \leq 2 y_{1}$
} 
(iii)

\begin{tabular}{|c|c|c|c|}
\hline 1. & $2 y_{2}$ & $\geq$ & $x_{2}$ \\
\hline 2. & $2 y_{1}$ & $<$ & $x_{1}$ \\
\hline 3. & $\mu_{1}+2 y_{2}-x_{2}$ & $\leq$ & $\underline{x}$ \\
\hline 4. & $\mu_{1}+2 y_{2}-x_{2}$ & $\leq$ & $\mu_{2}$ \\
\hline $5 .^{16}$ & $a_{13}$ & $\leq$ & $a_{23}$ \\
\hline 6. & $1-\left(\mu_{1}+2 y_{2}-x_{2}\right)+a_{13}$ & $\leq$ & $2 a_{23}$ \\
\hline 7. & $\frac{1}{2}\left(1-\mu_{2}+a_{23}\right)$ & $\leq$ & $1-\underline{x}$ \\
\hline 8. & $1-\left(2 y_{2}-x_{2}\right)+a_{13}$ & $>$ & $2 a_{23}$ \\
\hline 9. & $\frac{1}{3}\left(1-\left(2 y_{2}-x_{2}\right)+a_{13}+a_{23}\right)$ & $\leq$ & $1-\underline{x}$ \\
\hline
\end{tabular}

- Drawing lots versus $D \frac{x}{3}$ :

The same argumentation holds as for constraint set (i) of $D_{3}^{\mu_{i}}$.

- Correlated equilibrium versus $D_{3}^{\underline{x}}$ :

The same argumentation holds as for constraint sets (i) and (ii) of $D_{\frac{x}{3}}$

- Simplex minimization for Player 3:

$$
\pi_{3}^{\min }\left(D_{3}^{\underline{x}}(\mathrm{iii})\right)=\frac{3}{8}
$$

- Examples

$$
\begin{aligned}
& \text { General example: } \quad\left(\begin{array}{ccc}
0.6 & 0.38 & 0.02 \\
0.1 & 0.61 & 0.29 \\
0.6 & 0.00 & 0.40
\end{array}\right) \\
& \text { Minium payoff example: } \quad\left(\begin{array}{ccc}
\frac{5}{8} & \frac{3}{8} & 0 \\
\frac{1}{8} & \frac{5}{8} & \frac{2}{8} \\
\frac{5}{8} & 0 & \frac{3}{8}
\end{array}\right)
\end{aligned}
$$

\begin{tabular}{|l|rc|}
\hline 1. & $2 y_{2}$ & $\geq x_{2}$ \\
\hline 2. & $2 y_{1}$ & $\geq x_{1}$ \\
\hline 3. & $\mu_{1}+2 y_{2}-x_{2}$ & $\geq \underline{x}$ \\
\hline 4. & $\mu_{2}+2 y_{1}-x_{1}$ & $\geq \underline{x}$ \\
\hline
\end{tabular}

- Drawing lots versus $D^{\underline{x}}$ :

$u_{3}^{\max } \leq 1-\underline{x}$ follows directly from constraints (3.) and (4.).

- Correlated equilibrium versus $D^{\underline{x}}$ :

$\pi_{3}^{\max }$ (Correlated equilibrium) $\leq 1-\underline{x}$ follows directly from constraints (3.) and (4.).

\footnotetext{
${ }^{16}$ Note that $a_{23}<a_{13}$ is not possible. Suppose $a_{23}<a_{13} \Longrightarrow$ condition 6 . converts to $1-\left(\mu_{1}+\right.$ $\left.2 y_{2}-x_{2}\right)+a_{23} \leq 2 a_{13} \Longrightarrow x_{1}<y_{1}$
} 
- Simplex minimization for Player 3:

$$
\pi_{3}^{\min }\left(D_{\frac{x}{3}}(\mathrm{iv})\right)=\frac{3}{8}
$$

- Examples

$$
\begin{aligned}
& \text { General example: } \quad\left(\begin{array}{ccc}
0.60 & 0.40 & 0.0 \\
0.35 & 0.65 & 0.0 \\
0.60 & 0.00 & 0.4
\end{array}\right) \\
& \text { Minium payoff example: } \quad\left(\begin{array}{ccc}
\frac{5}{8} & \frac{3}{8} & 0 \\
\frac{3}{8} & \frac{5}{8} & 0 \\
\frac{5}{8} & 0 & \frac{3}{8}
\end{array}\right)
\end{aligned}
$$

\begin{tabular}{|c|c|c|c|}
\hline 1. & $2 y_{2}$ & $\geq$ & $x_{2}$ \\
\hline 2. & $2 y_{1}$ & $<$ & $x_{1}$ \\
\hline 3. & $\mu_{2}$ & $\leq$ & $\underline{x}$ \\
\hline 4. & $\mu_{1}+2 y_{2}-x_{2}$ & $\geq$ & $\mu_{2}$ \\
\hline $5 .^{17}$ & $a_{13}$ & $\leq$ & $a_{23}$ \\
\hline 6. & $1-\mu_{2}+a_{13}$ & $\leq$ & $2 a_{23}$ \\
\hline 7. & $\frac{1}{2}\left(1-\mu_{2}+a_{23}\right)$ & $<$ & $1-\underline{x}$ \\
\hline 8. & $1-\left(2 y_{2}-x_{2}\right)+a_{13}$ & $>$ & $2 a_{23}$ \\
\hline 9. & $\frac{1}{3}\left(1-\left(2 y_{2}-x_{2}\right)+a_{13}+a_{23}\right)$ & $\leq$ & $\frac{1}{2}\left(1-\mu_{2}+a_{23}\right)$ \\
\hline
\end{tabular}

5. Constraints on $D_{3}^{C o r}$ being the best proposal for Player 3:

(i)

- Drawing lots versus $D_{3}^{\text {cor }}$ : See constraint (9.).

- Simplex minimization for Player 3:

$$
\pi_{3}^{\min }\left(D_{3}^{\text {cor }}(i)\right)>\frac{3}{8}
$$

- Examples

$$
\begin{aligned}
& \text { General example: } \quad\left(\begin{array}{ccc}
0.58 & 0.42 & 0.00 \\
0.06 & 0.58 & 0.36 \\
0.00 & 0.50 & 0.50
\end{array}\right) \\
& \text { Minium payoff example: } \quad\left(\begin{array}{ccc}
\frac{5}{8}-\epsilon & \frac{3}{8}+\epsilon & 0 \\
\frac{1}{8}-5 \epsilon & \frac{5}{8}-\epsilon & \frac{1}{4}+6 \epsilon \\
0 & \frac{1}{2} & \frac{1}{2}
\end{array}\right)
\end{aligned}
$$

\footnotetext{
${ }^{17}$ See footnote 15 .
} 
(ii)

\begin{tabular}{|c|c|c|c|}
\hline 1. & $2 y_{2}$ & $\geq$ & $x_{2}$ \\
\hline 2. & $2 y_{1}$ & $<$ & $x_{1}$ \\
\hline 3. & $\mu_{1}+2 y_{2}-x_{2}$ & $<$ & $\underline{x}$ \\
\hline 4. & $\mu_{1}+2 y_{2}-x_{2}$ & $<$ & $\mu_{2}$ \\
\hline $5 . .^{18}$ & $a_{13}$ & $\leq$ & $a_{23}$ \\
\hline 6. & $1-\left(\mu_{1}+2 y_{2}-x_{2}\right)+a_{13}$ & $\leq$ & $2 a_{23}$ \\
\hline 7. & $\frac{1}{2}\left(1-\mu_{2}+a_{23}\right)$ & $>$ & $1-\underline{x}$ \\
\hline 8. & $1-\left(2 y_{2}-x_{2}\right)+a_{13}$ & $>$ & $2 a_{23}$ \\
\hline 9. & $\frac{1}{3}\left(1-\left(2 y_{2}-x_{2}\right)+a_{13}+a_{23}\right)$ & $\leq$ & $\frac{1}{2}\left(1-\mu_{2}+a_{23}\right)$ \\
\hline
\end{tabular}

- Drawing lots versus $D_{3}^{c o r}$ : See constraint (9.).

- Simplex minimization for Player 3:

$$
\pi_{3}^{\min }\left(D_{3}^{c o r}(i)\right)>\frac{3}{8}
$$

- Examples:

$$
\begin{aligned}
& \text { General example: } \quad\left(\begin{array}{ccc}
0.61 & 0.39 & 0.00 \\
0.04 & 0.65 & 0.31 \\
0.00 & 0.52 & 0.48
\end{array}\right) \\
& \text { Minium payoff example: } \quad\left(\begin{array}{ccc}
\frac{5}{8}+\epsilon & \frac{3}{8}-\epsilon & 0 \\
\frac{1}{8}-7 \epsilon & \frac{5}{8}+\epsilon & \frac{1}{4}+6 \epsilon \\
0 & \frac{1}{2} & \frac{1}{2}
\end{array}\right)
\end{aligned}
$$

\begin{tabular}{|c|c|c|c|}
\hline 1. & $2 y_{2}$ & $\geq$ & $x_{2}$ \\
\hline 2. & $2 y_{1}$ & $<$ & $x_{1}$ \\
\hline 3. & $\mu_{1}+2 y_{2}-x_{2}$ & $\leq$ & $\underline{x}$ \\
\hline 4. & $\mu_{1}+2 y_{2}-x_{2}$ & $\leq$ & $\mu_{2}$ \\
\hline $5^{19}$ & $a_{13}$ & $\leq$ & $a_{23}$ \\
\hline 6. & $1-\left(\mu_{1}+2 y_{2}-x_{2}\right)+a_{13}$ & $\leq$ & $2 a_{23}$ \\
\hline 7. & $\frac{1}{2}\left(1-\mu_{2}+a_{23}\right)$ & $<$ & $\frac{1}{3}\left(1-\left(2 y_{2}-x_{2}\right)+a_{13}+a_{23}\right)$ \\
\hline 8. & $1-\left(2 y_{2}-x_{2}\right)+a_{13}$ & $>$ & $2 a_{23}$ \\
\hline 9. & $\frac{1}{3}\left(1-\left(2 y_{2}-x_{2}\right)+a_{13}+a_{23}\right)$ & $>$ & $1-\underline{x}$ \\
\hline
\end{tabular}

6. $D_{3}^{T}$

Constraints on $D_{3}^{T}$ being the best proposal for Player 3:

- Drawing lots versus $D_{3}^{c o r}$ : See constraint (9.).

\footnotetext{
${ }^{18}$ See footnote 16 .

${ }^{19}$ See footnote 16 .
} 
- Simplex minimization for Player 3:

$$
\pi_{3}^{\min }\left(D_{3}^{L}\right)>\frac{3}{8}
$$

- Examples

$$
\begin{aligned}
& \text { General example: } \quad\left(\begin{array}{ccc}
0.64 & 0.36 & 0.00 \\
0.08 & 0.64 & 0.28 \\
0.00 & 0.08+\epsilon & 0.92-\epsilon
\end{array}\right) \quad(\epsilon<0.01) \\
& \text { Minium payoff example: } \quad\left(\begin{array}{ccc}
\frac{5}{8}+\epsilon & \frac{3}{8}-\epsilon & 0 \\
\frac{1}{8}-3 \epsilon & \frac{5}{8}+\epsilon & \frac{1}{4}+2 \epsilon \\
0 & \frac{1}{8}-3 \epsilon+\epsilon^{\prime} & \frac{7}{8}+3 \epsilon-\epsilon^{\prime}
\end{array}\right) \quad\left(\epsilon>3 \epsilon^{\prime}\right)
\end{aligned}
$$

Since the minimum payoff of $D_{\frac{x}{3}}$ is given by $\pi_{3}^{\min }\left(D_{3}^{\underline{x}}\right)=\frac{3}{8}$ and the constraints for $D_{3}^{\text {cor }}$ and $D_{3}^{L}$ directly imply that $\pi_{3}\left(D_{3}^{\text {cor }}\right)>1-\underline{x}$ and $\pi_{3}\left(D_{3}^{L}\right)>1-\underline{x}$, we obtain $\pi_{3}^{\min }\left(D_{3}^{\text {cor }}\right)>\frac{3}{8}$ and $\pi_{3}^{\min }\left(D_{3}^{L}\right)>\frac{3}{8}$.

Altogether we obtain

$$
\min _{\alpha \in\left\{D_{3}^{\mu}, D_{3}^{\bar{\mu}}, D_{3}^{\mu_{i}}, D_{3}^{\frac{x}{x}}, D_{3}^{C o r}, D_{3}^{L}\right\}}\left\{\pi_{3}(\alpha)\right\}=\min _{\rho \text { symmetric }}\left\{\pi_{3}\right\}=\frac{3}{8}
$$

\subsubsection{Proof of Corollary 1}

Given $a=\left(\begin{array}{ll}0 & 1 \\ 1 & 0\end{array}\right)$ the constraints on $D_{3}^{\underline{\mu}}$ hold when $\mu_{1}=\mu_{2}=\underline{\mu}=\frac{1}{2}$, which implies that $D_{3}=\left(\frac{1}{2}, 0, \frac{1}{2}\right)$ or $D_{3}=\left(0, \frac{1}{2}, \frac{1}{2}\right)$ are the best reactions for Player 3 resulting in the payoffs $\pi_{1}=\pi_{2}=\frac{1}{4}$ and $\pi_{3}=\frac{1}{2}$

By direct comparisons between the six possible proposals $\left(D_{3}^{\underline{\mu}}, D_{3}^{\bar{\mu}}, D_{3}^{\mu_{i}}, D_{3}^{\underline{x}}, D_{3}^{C o r}\right.$, $D_{3}^{L}$ ) for Player 3, we also obtain the following corollary, which will be used later:

Corollary 5

Given symmetric $\rho \Longrightarrow \pi_{2}>\underline{x}$ iff $D_{3}^{\text {cor }}$ or $D_{3}^{L}$ is the best reaction for Player 3 .

\subsubsection{Proof of Proposition 2}

There are six possibilities for $\rho$ to be non-symmetric:

$$
\begin{gathered}
\rho_{1}^{n s}=\left(\begin{array}{ll}
1 & 1 \\
2 & 2
\end{array}\right) \quad \rho_{1^{\prime}}^{n s}=\left(\begin{array}{ll}
2 & 2 \\
1 & 1
\end{array}\right) \\
\rho_{3}^{n s}=\left(\begin{array}{ll}
1 & 2 \\
2 & 2
\end{array}\right) \quad \rho_{3^{\prime}}^{n s}=\left(\begin{array}{ll}
2 & 1 \\
2 & 2
\end{array}\right) \quad \rho_{3^{\prime \prime}}^{n s}=\left(\begin{array}{ll}
2 & 2 \\
2 & 1
\end{array}\right) \quad \rho_{3^{\prime \prime \prime}}^{n s}=\left(\begin{array}{ll}
2 & 2 \\
1 & 2
\end{array}\right)
\end{gathered}
$$


In the following we only discuss $\rho_{1}^{n s}$, as the other cases arise by interchanging rows and observing that for $\rho_{3}^{n s}$. nothing changes qualitatively. For $\rho_{1}^{n s} A$ is given by

$$
A=\left(\begin{array}{ccc}
y_{1} & y_{2} & a_{13} \\
x_{1} & x_{2} & a_{23} \\
a_{31} & a_{32} & a_{33}
\end{array}\right) \quad \begin{aligned}
& a_{13}=1-y_{1}-y_{2} \\
& a_{23}=1-x_{1}-x_{2} \\
& a_{33}=1-a_{31}-a_{32}
\end{aligned}
$$

First we observe that Player 3 has to offer at least Player $i$ more than $x_{i}$ to gain more than $\pi_{3}=1-x_{1}-x_{2}$, because otherwise $D_{2}$ contains two maximum shares and is a single-proposal equilibrium. ${ }^{20}$

As in the proof of Proposition 1, we look for the best offer $D_{3}$ and see that the following properties of $a$ are relevant:

- $1 \geq a_{3 i}>2 x_{i}-y_{i} \Longrightarrow \Phi\left(x_{i}\right)=2, \Phi\left(y_{i}\right)=1, \Phi\left(a_{3 i}\right)=3$

- $y_{i}>a_{3 i}>2 y_{i}-x_{i} \Longrightarrow \Phi\left(x_{i}\right)=3, \Phi\left(y_{i}\right)=2, \Phi\left(a_{3 i}\right)=1$

With these properties we can distinguish four different kinds of proposals $D_{3}$ discussed in detail below:

$$
D_{3}^{x^{\epsilon}} \quad D_{3}^{o} \quad D_{3}^{3} \quad D_{3}^{L^{\epsilon}}
$$

1. $D^{\underline{x}^{\epsilon}}$

Player 3 offers Player 1 or $2 \underline{x}+\epsilon$ and keeps the rest for himself, such that $D_{3}$ is a single-proposal equilibrium and he receives a payoff $\pi_{3}=1-\underline{x}-\epsilon$ (i.e):

$$
\begin{aligned}
\left(\begin{array}{ccc}
y_{1} & y_{2} & a_{13} \\
x_{1} & x_{2} & a_{23} \\
\underline{x}+\epsilon & 0 & 1-\underline{x}-\epsilon
\end{array}\right) \longrightarrow\left(\begin{array}{ccc}
1 & 2 & 1 \\
2^{*} & 3 & 2 \\
3 & 1 & 3
\end{array}\right) ; \\
D_{1}=\left(\begin{array}{llll}
0.2 & 0.4 & 0.4
\end{array}\right) \\
D_{2}=\left(\begin{array}{llll}
0.4 & 0.6 & 0.0
\end{array}\right) \\
D_{\underline{x}^{\epsilon}}=\left(\begin{array}{lll}
0.4+\epsilon & 0.0 & 0.6-\epsilon
\end{array}\right)
\end{aligned}
$$

2. $D_{3}^{o}$

Player 3 offers Player $i a_{3 i}=2 x_{i}-y_{i}+\epsilon$ and keeps the rest for himself, such that $D_{1}$ is a single-proposal equilibrium and he receives a payoff $\pi_{3}=1-y_{1}-y_{2}$, i.e.

$$
\begin{aligned}
\left(\begin{array}{ccc}
y_{1} & y_{2} & a_{13} \\
x_{1} & x_{2} & a_{23} \\
2 x_{1}-y_{1}+\epsilon & 0 & 1-\left(2 x_{1}-y_{1}+\epsilon\right.
\end{array}\right) \longrightarrow\left(\begin{array}{ccc}
1 & 2^{*} & 3 \\
2 & 3 & 2 \\
3 & 1 & 1
\end{array}\right) \\
D_{1}=\left(\begin{array}{lll}
0.1 & 0.25 & 0.65
\end{array}\right) \\
D_{2}=\left(\begin{array}{lll}
0.5 & 0.40 & 0.10
\end{array}\right) \\
D_{3}^{o}=\left(\begin{array}{lll}
0.9+\epsilon & 0.00 & 0.1-\epsilon
\end{array}\right)
\end{aligned}
$$

\footnotetext{
${ }^{20}$ If Player 3 only offers Player $1 x_{1}, D_{2}$ is still the only proposal with two maxima, or $D_{2}$ and $D_{3}$ are both voting equilibria but are not correlated.
} 
3. $D_{3}^{3}$

Player 3 offers Player $i 2 x_{i}-y_{i}+\epsilon(i \in\{1,2\})$ and keeps the rest for himself, such that $D_{3}$ is a single-proposal equilibrium and he receives a payoff $\pi_{3}=1-$ $\left(2 x_{i}-y_{i}+\epsilon\right)$, i.e.

$$
\begin{gathered}
\left(\begin{array}{ccc}
y_{1} & y_{2} & a_{13} \\
x_{1} & x_{2} & a_{23} \\
2 x_{1}-y_{1}+\epsilon & 0 & 1-\left(2 x_{1}-y_{1}+\epsilon\right.
\end{array}\right) \longrightarrow\left(\begin{array}{ccc}
1 & 2 & 3 \\
2 & 3 & 1 \\
3 & 1 & 2^{*}
\end{array}\right) \\
D_{1}=\left(\begin{array}{lll}
0.1 & 0.25 & 0.65
\end{array}\right) \\
D_{2}=\left(\begin{array}{lll}
0.3 & 0.60 & 0.10
\end{array}\right) \\
D_{3}^{3}=\left(\begin{array}{lll}
0.5+\epsilon & 0.00 & 0.5-\epsilon
\end{array}\right)
\end{gathered}
$$

4. $D_{3}^{T^{\epsilon}}$

Player 3 offers Player $i a_{3 i}=2 x_{i}-y_{i}+\epsilon$ and keeps the rest for himself, such that the proposal is chosen by drawing lots and he receives a payoff $\pi_{3}=\frac{1}{3}\left(1-\left(2 x_{1}-\right.\right.$ $\left.\left.y_{1}+\epsilon\right)+a_{13}+a_{23}\right)$, i.e.:

$$
\begin{gathered}
\left(\begin{array}{ccc}
y_{1} & y_{2} & a_{13} \\
x_{1} & x_{2} & a_{23} \\
2 x_{1}-y_{1}+\epsilon & 0 & 1-\left(2 x_{1}-y_{1}+\epsilon\right.
\end{array}\right) \rightarrow\left(\begin{array}{lll}
1 & 2 & 3 \\
2 & 3 & 1 \\
3 & 1 & 2
\end{array}\right) \\
D_{1}=\left(\begin{array}{lll}
0.1 & 0.1 & 0.8
\end{array}\right) \\
D_{2}=\left(\begin{array}{lll}
0.4 & 0.6 & 0.0
\end{array}\right) \\
D_{3}^{T^{\epsilon}}=\left(\begin{array}{lll}
0.7+\epsilon & 0.0 & 0.3-\epsilon
\end{array}\right)
\end{gathered}
$$

1. $D^{\underline{x}^{\epsilon}}$

Constraints on $D_{\frac{x^{\epsilon}}{3}}$ being the best proposal for Player 3:

$$
\text { 1. } y_{1}+y_{2}>\underline{x}
$$

Since Player 3 needs to offer at least Player $i a_{3 i}=\underline{x}+\epsilon$ to prevent $D_{2}$ from being a single-proposal equilibrium and $1-\underline{x}+\epsilon>\bar{a}_{i 3} i=1,2, D_{3}^{\underline{x}}$ is better than all other proposals.

- Simplex minimization for Player 3:

$$
\pi_{3}^{\min }\left(D_{3}^{x^{\epsilon}}\right)>\frac{1}{2}
$$

General example: $\quad\left(\begin{array}{ccc}0.2 & 0.4 & 0.4 \\ 0.4 & 0.6 & 0.0 \\ 0.4+\epsilon & 0.0 & 0.6-\epsilon\end{array}\right)$

Minium payoff example: $\left(\begin{array}{ccc}\frac{1}{4}-3 \epsilon & \frac{1}{4}+\epsilon & \frac{1}{2}+2 \epsilon \\ \frac{1}{2}-4 \epsilon & \frac{1}{2}+4 \epsilon & 0 \\ \frac{1}{2}-4 \epsilon+\epsilon^{\prime} & 0 & \frac{1}{2}+4 \epsilon-\epsilon^{\prime}\end{array}\right) \epsilon^{\prime}<\epsilon$ 
2. $D_{3}^{o}$

Constraints on $D_{3}^{o}$ being the best proposal for Player 3:

$\left.\begin{array}{|l|rl|}\hline 1 . & y_{1}+y_{2} & \leq \\ \hline 2 . & 2 y_{i} & \geq \\ \hline 3 . & 2 x_{j}-y_{j} & <1\end{array}\right\} i, j=1,2 i \neq j$

Note that $r_{23}=2^{*}$ is not possible, as we have

$$
2 x_{j}>y_{j}+\epsilon \Longleftrightarrow \underbrace{1-\left(2 x_{j}-y_{j}+\epsilon\right)}_{a_{33}\left(D_{3}^{o}\right)}+\underbrace{1-\left(y_{1}+y_{2}\right)}_{a_{13}}>\underbrace{2\left(1-\left(x_{1}+x_{2}\right)\right)}_{23}
$$

and if $a_{33}\left(D_{3}^{o}\right)=1-\left(2 x_{1}-y_{1}+\epsilon\right)>a_{23}$, Player 3 can rise $a_{j 3}$ so that $1-a_{3 j}=a_{23}$.

Since $\pi_{3}=1-y_{1}-y_{2}$ is only $\epsilon$ worse than the payoff of $D_{3}^{x^{\epsilon}}, D_{3}^{o}$ is the best offer.

- Simplex minimization for Player 3:

$$
\pi_{3}^{\min }\left(D_{3}^{o}\right)=\frac{1}{2}
$$

General example: $\quad\left(\begin{array}{ccc}0.1 & 0.25 & 0.65 \\ 0.5 & 0.40 & 0.10 \\ 0.9+\epsilon & 0.0 & 0.1-\epsilon\end{array}\right)$

Minium payoff example:

$$
\left(\begin{array}{ccc}
\frac{1}{4} & \frac{1}{4} & \frac{1}{2} \\
\frac{1}{2} & \frac{1}{2} & 0 \\
0 & \frac{3}{4}+\epsilon & \frac{1}{4}-\epsilon
\end{array}\right)
$$

3. $D_{3}^{3}$

Constraints for $D_{3}^{3}$ being the best proposal for Player 3:

(i)

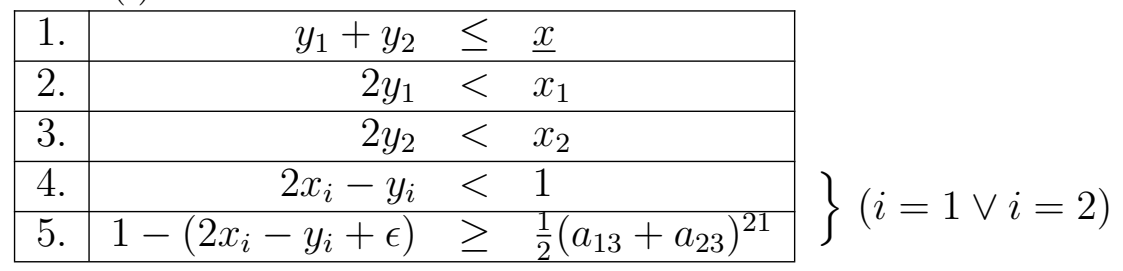

- Drawing lots versus $D_{3}^{3}$ :

With drawing lots, Player 3 cannot gain more than $\pi_{3}=\frac{1}{2}\left(a_{13}+a_{23}\right)-\epsilon^{\prime} \leq$ $\pi_{3}\left(D_{3}^{3}(i)\right)$ because of constraint (5.).

\footnotetext{
${ }^{21}$ If $2 x_{i}-y_{i}<1$ and $1-\left(2 x_{i}-y_{i}+\epsilon\right) \geq \frac{1}{2}\left(a_{13}+a_{23}\right)$ holds simultaneously for $i=1,2$, Player 3 chooses $\min _{i=1,2}\left\{2 x_{i}-y_{i}\right\}$.
} 
- Simplex minimization for Player 3:

$$
\pi_{3}^{\min }\left(D_{3}^{3}(i)\right)>\frac{1}{4}
$$

$$
\text { General example: } \quad\left(\begin{array}{ccc}
0.1 & 0.25 & 0.65 \\
0.3 & 0.60 & 0.10 \\
0.5+\epsilon & 0.0 & 0.5-\epsilon
\end{array}\right)
$$

Minium payoff example:

$$
\left(\begin{array}{ccc}
\frac{1}{4} & \frac{1}{4}-\epsilon & \frac{1}{2}+\epsilon \\
\frac{1}{2}+\epsilon & \frac{1}{2}-\epsilon & 0 \\
0 & \frac{3}{4}-\epsilon+\epsilon^{\prime} & \frac{1}{4}+\epsilon-\epsilon^{\prime}
\end{array}\right)
$$

\begin{tabular}{|c|c|c|c|}
\hline 1. & $y_{1}+y_{2}$ & $\underline{x}$ & \\
\hline 2. & $2 y_{i}$ & $x_{i}$ & \\
\hline 3. & $2 y_{j}$ & $x_{j}$ & \\
\hline 4. & $2 x_{j}-y_{j}$ & 1 & $i, j=1,2 i \neq j$ \\
\hline 5. & $2 x_{i}-y_{i}$ & 1 & \\
\hline 6 . & $1-\left(2 x_{j}-y_{j}+\epsilon\right)$ & $\frac{1}{2}\left(a_{13}+a_{23}\right)$ & \\
\hline
\end{tabular}

(ii)

- Drawing lots versus $D_{3}^{3}$ :

With drawing lots, Player 3 cannot gain more than $\pi_{3}=\frac{1}{2}\left(a_{13}+a_{23}\right)-\epsilon^{\prime} \leq$ $\pi_{3}\left(D_{3}^{3}(i i)\right)$ because of constraint (6.).

- Simplex minimization for Player 3:

$$
\pi_{3}^{\min }\left(D_{3}^{3}(i i)\right)>\frac{1}{3}
$$

General example: $\quad\left(\begin{array}{ccc}0.00 & 0.40 & 0.60 \\ 0.55 & 0.45 & 0.00 \\ 0.00 & 0.5+\epsilon & 0.5-\epsilon\end{array}\right)$

Minium payoff example:

$$
\left(\begin{array}{ccc}
0 & \frac{1}{3}+\epsilon & \frac{2}{3}-\epsilon \\
\frac{1}{2} & \frac{1}{2} & 0 \\
0 & \frac{2}{3}-\epsilon+\epsilon^{\prime} & \frac{1}{3}+\epsilon-\epsilon^{\prime}
\end{array}\right)
$$

4. $D_{3}^{T^{\epsilon}}$

Constraints on $D_{3}^{T^{\epsilon}}$ being the best proposal for Player 3: 
(i)

\begin{tabular}{|l|rl|}
\hline 1. & $y_{1}+y_{2}$ & $\leq \underline{x}$ \\
\hline 2. & $2 y_{1}$ & $<x_{1}$ \\
\hline 3. & $2 y_{2}$ & $<x_{2}$ \\
\hline 4. & $2 x_{i}-y_{i}$ & $<1$ \\
\hline 5. & $1-\left(2 x_{i}-y_{i}+\epsilon\right)$ & $<\frac{1}{2}\left(a_{13}+a_{23}\right)$ \\
\hline 6. & $1-\left(2 x_{j}-y_{j}+\epsilon\right)$ & $<\frac{1}{2}\left(a_{13}+a_{23}\right)$ \\
\hline 7. & $1-\left(2 x_{j}-y_{j}\right)$ & $\leq 1-\left(2 x_{i}-y_{i}\right)$ \\
\hline
\end{tabular}

- Since all other kinds of proposal are excluded, $D_{3}^{L^{\epsilon}}$ is the best proposal.

- Simplex minimization for Player 3:

$$
\pi_{3}^{\min }\left(D_{3}^{T^{\epsilon}}(i)\right)>\frac{1}{4}
$$

$$
\text { General example: } \quad\left(\begin{array}{ccc}
0.1 & 0.10 & 0.80 \\
0.4 & 0.6 & 0.00 \\
0.7+\epsilon & 0.0 & 0.3-\epsilon
\end{array}\right)
$$

Minium payoff example:

$$
\left(\begin{array}{ccc}
\frac{1}{4}-\epsilon & \frac{1}{4}-\epsilon & \frac{1}{2}+2 \epsilon \\
\frac{1}{2} & \frac{1}{2} & 0 \\
0 & \frac{3}{4}-\epsilon+\epsilon^{\prime} & \frac{1}{4}+\epsilon-\epsilon^{\prime}
\end{array}\right)
$$

\begin{tabular}{|c|c|c|c|}
\hline 1. & $y_{1}+y_{2} \leftrightharpoons$ & $\underline{x}$ & \\
\hline 2. & $2 y_{i}$ & $x_{i}$ & \\
\hline 3. & $2 y_{j}$ & $x_{j}$ & \\
\hline 4. & $2 x_{j}-y_{j}$ & 1 & $i, j=1,2 i \neq j$ \\
\hline 5. & $2 x_{i}-y_{i}$ & 1 & \\
\hline 6. & $1-\left(2 x_{j}-y_{j}+\epsilon\right)$ & $\frac{1}{2}\left(a_{13}+a_{23}\right)$ & \\
\hline
\end{tabular}

(ii)

- Since all other kinds of proposal are excluded, $D_{3}^{L^{\epsilon}}$ is the best proposal.

- Simplex minimization for Player 3:

$$
\pi_{3}^{\min }\left(D_{3}^{T^{\epsilon}}(i i)\right) \geq \frac{1}{3}-\epsilon
$$

General example: $\quad\left(\begin{array}{ccc}0.00 & 0.30 & 0.80 \\ 0.51 & 0.49 & 0.00 \\ 0.00 & 0.68+\epsilon & 0.32-\epsilon\end{array}\right)$

Minium payoff example:

$$
\left(\begin{array}{ccc}
0 & \frac{1}{3} & \frac{2}{3} \\
\frac{1}{2} & \frac{1}{2} & 0 \\
0 & \frac{2}{3}+\epsilon & \frac{1}{3}-\epsilon
\end{array}\right)
$$


Calculating the feasible set of $\left(D_{3}^{x^{\epsilon}} \cup D_{3}^{o} \cup D_{3}^{3} \cup D_{3}^{T^{\epsilon}}\right)$, we find that

\begin{tabular}{|l|rc|}
\hline 1. & $y_{1}+y_{2} \leq \underline{x}$ \\
\hline 2. & $2 y_{1}$ & $<x_{2}$ \\
\hline 3. & $2 y_{2}<x_{2}$ \\
\hline 4. & $2 x_{1}-y_{1} \geq 1$ \\
\hline 5. & $2 x_{2}-y_{2} \geq 1$ \\
\hline
\end{tabular}

is missing. The only combination of $\left(D_{1}, D_{2}\right)$ to fulfill this condition is given by

$A=\left(\begin{array}{ccc}0 & 0 & 1 \\ \frac{1}{2} & \frac{1}{2} & 0 \\ a_{31} & a_{32} & a_{33}\end{array}\right)$

Note that this is also submatrix $a$, excluded in Proposition 2.

Altogether we obtain for $a \neq\left(\begin{array}{cc}0 & 0 \\ \frac{1}{2} & \frac{1}{2}\end{array}\right) \wedge a \neq\left(\begin{array}{cc}\frac{1}{2} & \frac{1}{2} \\ 0 & 0\end{array}\right)$

$$
\min _{\alpha \in\left\{D_{3}^{\frac{x}{\epsilon}}, D_{3}^{o}, D_{3}^{3}, D_{3}^{T^{\epsilon}}\right\}}\left\{\pi_{3}(\alpha)\right\}=\min _{\rho \text { non-symmetric }}\left\{\pi_{3}\right\}>\frac{1}{4}
$$

\subsubsection{Proof of Corollary 2}

W.l.o.g we assume $a=\left(\begin{array}{cc}0 & 0 \\ \frac{1}{2} & \frac{1}{2}\end{array}\right) \Longrightarrow$ Player 3 must offer Player $i$ at least $a_{3 i}=\mu_{i}$ and Player $j$ at least $a_{3 j}=x_{j}+\epsilon(i, j=1,2 i \neq j)$ to prevent $D_{2}$ from being a singleproposal equilibrium with $\pi_{3}=0 . \Longrightarrow D_{3}=\left(\frac{1}{4}, \frac{1}{2}+\epsilon, \frac{1}{4}-\epsilon\right)$ or $D_{3}=\left(\frac{1}{2}+\epsilon, \frac{1}{4}, \frac{1}{4}-\epsilon\right)$ with the correlated equilibrium $C_{23}$ is the best reaction for Player 3 , resulting in the payoffs $\pi_{1}=\pi_{2}=\frac{7}{16}+\frac{\epsilon}{4}$ and $\pi_{3}=\frac{1}{8}-\frac{\epsilon}{2}$ (given $D_{1}$ and $D_{2}$, this is also the worst situation for Player 3 ).

\subsection{Proof of Propositions 3, 4, and 5, and of Corollaries 3 and 4}

We prove these propositions and corollaries by constructing $D_{2}$ in such a way that we satisfy different constraint sets in the proofs of Propositions 1 and 2 . But since we have only shown Propositions 1 and 2 for representative matrices $a$, we sometimes have to interchange indices in the constraint sets to adapt them for the following proofs. 


\subsubsection{Proof of Proposition 3}

1. Suppose that $D_{1} \in \mathcal{A} \Longrightarrow$ Player 2's payoff will be at least $\pi_{2}>\frac{3}{8}$ and $\rho=$ $\left(\begin{array}{ll}2 & 1 \\ 1 & 2\end{array}\right)$, if he proposes $D_{2}=\left(\frac{1}{2}, \frac{1}{2}, 0\right), D_{2}=\left(a_{12}, a_{11}-\epsilon, a_{13}+\epsilon\right)$, or $D_{2}=$ $\left(\frac{1}{2}-\epsilon, \frac{1}{2}-\epsilon, 2 \epsilon\right)$.

$\left.\mathcal{A}_{1}\right)$ Suppose $D_{2}=\left(\frac{1}{2}, \frac{1}{2}, 0\right)$ and $D_{3}=\left(0, \frac{1}{2}, \frac{1}{2}\right)$ is the best reaction for Player 3 and a single-proposal equilibrium $\Longrightarrow$ This requires the following rank matrix:

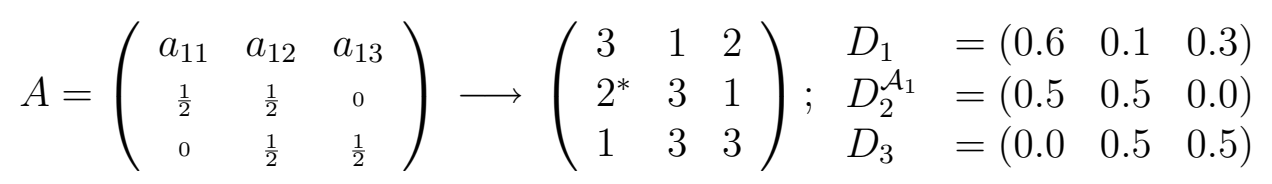

This can be satisfied if constraint set (i) of $D_{3}^{\underline{x}}$ holds. The feasible set $\mathcal{S}_{\mathcal{A}_{1}}$ is then determined by ${ }^{22}$

$$
\begin{aligned}
& \text { 1. } \quad 2 a_{21} \geq a_{11} \Longrightarrow a_{11} \leq 1 \\
& \text { 2. } \quad 2 a_{12}<a_{22} \Longrightarrow a_{12}<\frac{1}{4} \\
& \text { 3. } \quad \mu_{1}>\underline{x} \Longrightarrow a_{11}>\frac{1}{2} \\
& \text { 4. } \mu_{2}+2 y_{1}-x_{1} \geq \underline{x} \Longrightarrow a_{12} \geq 2 a_{11}-\frac{3}{2}
\end{aligned}
$$

After comparison of the inequalities, only 3. and 4. remain.

(See Figure 1)

$$
\Longrightarrow \mathcal{S}_{\mathcal{A}_{1}}=\left\{\left(\frac{1}{2}, \frac{3}{4}\right] \times\left[0, \frac{1}{4}\right] \cup\left[\frac{3}{4}, \frac{5}{6}\right] \times\left[2 a_{11}-\frac{3}{2}, 1-a_{11}\right]\right\}
$$

Minimum payoff for Player 2:

$$
\pi_{2}^{\min }\left(\mathcal{A}_{1}\right)=\frac{1}{2}
$$

$\left.\mathcal{A}_{2}\right)$ See $\mathcal{A}_{1}$, but now with constraint set (iv) of $D^{\underline{x}}$.

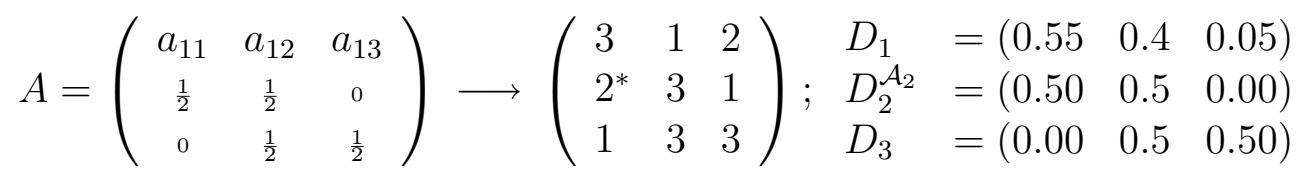

This requires

$$
\begin{array}{rr}
2 a_{21} & \geq a_{11} \Longrightarrow a_{11} \leq 1 \\
2 a_{12} & \geq a_{22} \Longrightarrow a_{12} \geq \frac{1}{4} \\
\text { 2. } & \Longrightarrow a_{12} \geq 2 a_{11}-\frac{3}{2} \\
\text { 3. } \mu_{2}+2 a_{21}-a_{11} & \geq \underline{x} \Longrightarrow a_{12} \geq \frac{3}{8}-\frac{1}{4} a_{11} \\
\text { 4. } \mu_{1}+2 a_{12}-a_{22} & \geq \underline{x} \Longrightarrow a_{11} \geq \frac{1}{2} \\
\text { 5. } & a_{11}
\end{array}
$$

\footnotetext{
${ }^{22}$ The bold numbers will denote those constraints which are binding.
} 
After comparison of the inequalities, only 2. and 5. remain.

$$
\Longrightarrow \mathcal{S}_{\mathcal{A}_{2}}=\left\{\left(\frac{1}{2}, \frac{3}{4}\right] \times\left[\frac{1}{4}, 1-a_{11}\right]\right\}
$$

(See Figure 1)

Minimum payoff for Player 2:

$$
\pi_{2}^{\min }\left(\mathcal{A}_{2}\right)=\frac{1}{2}
$$

$\left.\mathcal{A}_{3}\right)$ Suppose $D_{2}=\left(a_{12}, a_{11}-\epsilon, a_{13}+\epsilon\right)$ and $D_{3}=\left(0, \mu_{2}, 1-\mu_{2}\right)$ is a single-proposal equilibrium $\Longrightarrow$ This requires the following rank matrix:

$$
\begin{gathered}
A=\left(\begin{array}{lll}
a_{11} & a_{12} & a_{13} \\
a_{12} & a_{11}-\epsilon & a_{13}+\epsilon \\
0 & \mu_{2} & 1-\mu_{2}
\end{array}\right) \longrightarrow\left(\begin{array}{lll}
3 & 1 & 1 \\
2 & 3 & 2 \\
1 & 2^{*} & 3
\end{array}\right) ; \\
D_{1}=\left(\begin{array}{lll}
0.90 & 0.05 & 0.05
\end{array}\right) \\
D_{2}^{\mathcal{A}_{3}}=\left(\begin{array}{lll}
0.05 & 0.9-\epsilon & 0.05+\epsilon
\end{array}\right) \\
D_{3}=\left(\begin{array}{lll}
0.00 & 0.45-\frac{\epsilon}{2} & 0.55+\frac{\epsilon}{2}
\end{array}\right)
\end{gathered}
$$

This can be satisfied if the constraint set of $D_{3}^{\underline{\mu}}$ holds (note that $\mu_{2}=\mu$ ). The feasible set $\mathcal{S}_{\mathcal{A}_{3}}$ is then determined by

$$
\begin{aligned}
& \text { 1. } 2 a_{21}<a_{11} \Longrightarrow a_{11}>\frac{3}{4} \\
& \text { 2. } a_{23} \geq a_{13} \Longrightarrow \epsilon>0 \\
& \text { 3. } 1-\mu_{2}+a_{13}>2 a_{23} \Longrightarrow a_{12} \geq \epsilon^{\prime}-a_{11} \\
& \text { 4. } \mu_{2}>\frac{3}{8} \Longrightarrow a_{12}>\frac{3}{4}-a_{11}
\end{aligned}
$$

After comparison of the inequalities, constraints 1. and 4. remain.

$$
\Longrightarrow \mathcal{S}_{\mathcal{A}_{3}}=\left\{\left(\frac{3}{4}, 1\right] \times\left[0,1-a_{11}\right]\right\}
$$

(See Figure 1)

Minimum payoff for Player 2:

$$
\pi_{2}^{\min }\left(\mathcal{A}_{3}\right)>\frac{3}{8}
$$

$\mathcal{A}_{4}$ ) To obtain complete cover for $\mathcal{A}$ there remains the line given by $a_{11}=\frac{1}{2}$. Suppose $D_{2}=\left(\frac{1}{2}-\epsilon, \frac{1}{2}-\epsilon, 2 \epsilon\right)\left(\epsilon<\frac{1}{2}-a_{12}\right)$ and $D_{3}=\left(0, \frac{1}{2}-\epsilon, \frac{1}{2}+\epsilon\right)$ is the best reaction for Player 3 and a single-proposal equilibrium $\Longrightarrow$ This requires the following rank matrix:

$$
A=\left(\begin{array}{ccc}
\frac{1}{2} & a_{12} & a_{13} \\
\frac{1}{2}-\epsilon & \frac{1}{2}-\epsilon & 2 \epsilon \\
0 & \frac{1}{2}-\epsilon & \frac{1}{2}+\epsilon
\end{array}\right) \quad \longrightarrow \quad\left(\begin{array}{ccc}
3 & 1 & 2 \\
2^{*} & 3 & 1 \\
1 & 3 & 3
\end{array}\right)
$$




$$
\begin{aligned}
& D_{1}=\left(\begin{array}{lcc}
0.5 & 0.4 & 0.1
\end{array}\right) \\
& D_{2}^{\mathcal{A}_{4}}=\left(\begin{array}{llc}
0.5-\epsilon & 0.5-\epsilon & 2 \epsilon
\end{array}\right) \\
& D_{3}=\left(\begin{array}{lll}
0.00 & 0.5-\epsilon & 0.5+\epsilon
\end{array}\right)
\end{aligned}
$$

Taking $a_{11}=\frac{1}{2}, a_{21}=a_{22}=\frac{1}{2}-\epsilon$, we see that either of the constraints on $\mathcal{A}_{1}$ or $\mathcal{A}_{2}$ hold and thus we obtain

$$
\Longrightarrow \mathcal{S}_{\mathcal{A}_{4}}=\left\{\left[\frac{1}{2}, \frac{1}{2}\right] \times\left[0, \frac{1}{2}\right]\right\}
$$

(See Figure 1)

Minimum payoff for Player 2:

$$
\pi_{2}^{\min }\left(\mathcal{A}_{4}\right)=\frac{1}{2}-\epsilon
$$

2. Suppose $D_{1} \in \mathcal{B} \Longrightarrow$ Player 2's payoff will be at least $\pi_{2}>\frac{3}{8}$ and $\rho=\left(\begin{array}{ll}1 & 2 \\ 2 & 1\end{array}\right)$, if he proposes $D_{2}=\left(a_{12}+\epsilon, 1-\left(a_{12}+\epsilon\right), 0\right)$,

$D_{2}=\left(\frac{3}{4}-a_{11}+3 \epsilon, \frac{3}{4}-a_{12}+2 \epsilon, a_{11}+a_{12}-\frac{1}{2}-5 \epsilon\right), D_{2}=\left(a_{12}+\epsilon, 1-\left(a_{12}+\epsilon\right), 0\right)$, $D_{2}=\left(a_{12}+\frac{1}{4}-2 \epsilon, \frac{3}{4}-a_{12}+2 \epsilon, 0\right), D_{2}=\left(\frac{5}{8}+4 \epsilon, \frac{3}{8}-4 \epsilon, 0\right)$,

$D_{2}=\left(a_{12}+4 \epsilon, 1-\left(a_{12}+4 \epsilon\right), 0\right)$ or $D_{2}=(1,0,0)$.

$\left.\mathcal{B}_{1}\right)$ Suppose $D_{2}=\left(a_{12}+\epsilon, 1-\left(a_{12}+\epsilon\right), 0\right)$ and $D_{3}=\left(0, a_{12}, 1-a_{12}\right)$ is the best reaction for Player 3 and a single-proposal equilibrium $\Longrightarrow$ This requires the following rank matrix:

$$
\begin{gathered}
A=\left(\begin{array}{lll}
a_{11} & a_{12} & a_{13} \\
a_{12}+\epsilon & 1-\left(a_{12}+\epsilon\right) & 0 \\
0 & a_{12} & 1-a_{12}
\end{array}\right) \longrightarrow\left(\begin{array}{lll}
2 & 3 & 2 \\
3 & 1 & 1 \\
1 & 3 & 3
\end{array}\right) \\
D_{1}=\left(\begin{array}{lll}
0.1 & 0.55 & 0.35
\end{array}\right) \\
D_{2}^{\mathcal{B}_{1}}=\left(\begin{array}{lll}
0.55+\epsilon & 0.45-\epsilon & 0
\end{array}\right) \\
D_{3}=\left(\begin{array}{lll}
0.00 & 0.55 & 0.45
\end{array}\right)
\end{gathered}
$$

This can be satisfied if the constraint set (ii) of $D_{3}^{\underline{x}}$ holds (by construction we have $\underline{x}=a_{12}$ ). The feasible set $\mathcal{S}_{\mathcal{B}_{1}}$ is then determined by

$$
\begin{aligned}
& \text { 1. } 2 a_{22} \geq a_{12} \Longrightarrow a_{12}<\frac{2}{3} \\
& \text { 2. } 2 a_{11}<a_{21} \Longrightarrow a_{12} \geq 2 a_{11} \\
& \text { 3. } \quad \mu_{2}<\underline{x} \quad \Longrightarrow a_{12} \geq \frac{1}{2} \\
& \text { 4. } \mu_{1}+2 a_{22}-a_{12} \geq \mu_{2} \Longrightarrow a_{12}<\frac{3}{5}+\frac{a_{11}}{5} \\
& \text { 5. } \quad a_{23}<a_{13} \Longrightarrow a_{12}<1-a_{11} \\
& \text { 6. } 1-\mu_{2}+a_{23} \leq 2 a_{13} \Longrightarrow a_{12}<\frac{3}{4}-a_{11} \\
& \text { 7. } \frac{1}{2}\left(1-\mu_{2}+a_{13}\right) \leq 1-\underline{x} \Longrightarrow a_{12}<\frac{1}{2}+a_{11}
\end{aligned}
$$

After comparison of the inequalities, only 3., 6., and 7. remain.

$$
\Longrightarrow \mathcal{S}_{\mathcal{B}_{1}}=\left\{\left[0, \frac{1}{8}\right] \times\left[\frac{1}{2}, \frac{1}{2}+a_{11}\right) \cup\left[\frac{1}{8}, \frac{1}{4}\right] \times\left[\frac{1}{2}, \frac{3}{4}-a_{11}\right)\right\}
$$


(See Figure 2)

Minimum payoff for Player 2:

$$
\pi_{2}^{\min }\left(\mathcal{B}_{1}\right)=\frac{1}{2}
$$

$\left.\mathcal{B}_{2}\right)$ Suppose $D_{2}=\left(\frac{3}{4}-a_{11}+3 \epsilon, \frac{3}{4}-a_{12}+2 \epsilon, a_{11}+a_{12}-\frac{1}{2}-5 \epsilon\right)$ and $D_{3}=\left(0, \mu_{2}, 1-\mu_{2}\right)$ is the best reaction for Player 3 and a single-proposal equilibrium $\Longrightarrow$ This requires the following rank matrix:

$$
\begin{gathered}
A=\left(\begin{array}{lll}
a_{11} & a_{12} & a_{13} \\
\frac{3}{4}-a_{11}+3 \epsilon & \frac{3}{4}-a_{12}+2 \epsilon & 1-a_{21}-a_{22} \\
0 & \mu_{2} & 1-\mu_{2}
\end{array}\right) \longrightarrow\left(\begin{array}{lll}
2 & 3 & 2 \\
3 & 1 & 1 \\
1 & 2^{*} & 3
\end{array}\right) \\
D_{1}=\left(\begin{array}{lll}
0.2 & 0.55 & 0.25
\end{array}\right) \\
D_{2}^{\mathcal{B}_{2}}=\left(\begin{array}{lll}
0.55+3 \epsilon & 0.2+2 \epsilon & 0
\end{array}\right) \\
D_{3}=\left(\begin{array}{lll}
0.00 & 0.375+\epsilon & 0.625-\epsilon
\end{array}\right)
\end{gathered}
$$

This can be satisfied if the constraints of $D \frac{\mu}{3}$ hold. The feasible set $\mathcal{S}_{\mathcal{B}_{2}}$ is then determined by

$$
\begin{aligned}
& \text { 1. } \quad \mu_{2}<\mu_{1} \Longrightarrow \epsilon>0 \\
& \text { 2. } 2 a_{11}<a_{21} \Longrightarrow a_{11} \leq \frac{1}{4} \\
& \text { 3. } \quad a_{23} \leq a_{13} \Longrightarrow a_{12} \leq \frac{3}{4}-a_{11} \\
& \text { 4. } 1-\mu_{2}+a_{23}>2 a_{13} \Longrightarrow a_{12}>\frac{5}{8}-a_{11}
\end{aligned}
$$

After comparison of the inequalities, only 3. and 4. remain.

$$
\Longrightarrow \mathcal{S}_{\mathcal{B}_{2}}=\left\{\left[0, \frac{1}{8}\right] \times\left(\frac{5}{8}-a_{11}, \frac{3}{4}-a_{11}\right] \cup\left(\frac{1}{8}, \frac{1}{4}\right] \times\left[\frac{1}{2}, \frac{3}{4}-a_{11}\right]\right\}
$$

(See Figure 2)

Minimum payoff for Player 2:

$$
\pi_{2}^{\min }\left(\mathcal{B}_{2}\right)>\frac{3}{8}
$$

$\left.\mathcal{B}_{3}\right)$ Suppose $D_{2}=\left(a_{12}+\epsilon, 1-\left(a_{12}+\epsilon\right), 0\right)$ and $D_{3}=\left(0, a_{12}, 1-a_{12}\right)$ is the best reaction for Player 3 and a single-proposal equilibrium $\Longrightarrow$ This requires the following rank matrix:

$$
\begin{gathered}
A=\left(\begin{array}{lll}
a_{11} & a_{12} & a_{13} \\
a_{12}+\epsilon & 1-\left(a_{12}+\epsilon\right) & 0 \\
0 & a_{12} & 1-a_{12}
\end{array}\right) \\
D_{1}=\left(\begin{array}{lll}
0.35 & 0.55 & 0.10
\end{array}\right) \\
D_{2}^{\mathcal{B}_{2}}=\left(\begin{array}{lll}
0.55+\epsilon & 0.45-\epsilon & 0.00
\end{array}\right) \\
D_{3}=\left(\begin{array}{lll}
0.00 & 0.55 & 0.45
\end{array}\right)
\end{gathered}
$$


This can be satisfied if the constraint set (iv) of $D^{\underline{x}}$ holds. The feasible set $\mathcal{S}_{\mathcal{B}_{3}}$ is then determined by

$$
\begin{aligned}
2 a_{22} & \geq a_{12} \Longrightarrow a_{12}<\frac{2}{3} \\
2 a_{11} & \geq a_{21} \Longrightarrow a_{12}<2 a_{11} \\
\text { 2. } & \Longrightarrow a_{12}<\frac{1}{7} a_{11}+\frac{4}{7} \\
\text { 3. } \mu_{1}+2 a_{22}-a_{12} & \geq a_{12} \Longrightarrow a_{12}<\frac{1}{4}+a_{11} \\
\text { 4. } \mu_{2}+2 a_{11}-a_{21} & \geq a_{12} \Longrightarrow a_{12}
\end{aligned}
$$

After comparison of the inequalities only 4. remains.

$$
\Longrightarrow \mathcal{S}_{\mathcal{B}_{3}}=\left\{\left[\frac{1}{4}, \frac{3}{8}\right] \times\left[\frac{1}{2}, \frac{1}{4}+a_{11}\right) \cup\left(\frac{3}{8}, \frac{1}{2}\right] \times\left[\frac{1}{2}, 1-a_{11}\right]\right\}
$$

(See Figure 2)

Minimum payoff for Player 2:

$$
\pi_{2}^{\min }\left(\mathcal{B}_{3}\right)=\frac{1}{2}
$$

$\left.\mathcal{B}_{4}\right)$ Suppose $D_{2}=\left(a_{12}-\frac{1}{4}-2 \epsilon, \frac{3}{4}-a_{12}+2 \epsilon, 0\right)$ and $D_{3}=\left(0, \mu_{2}, 1-\mu_{2}\right)$ is the best reaction for Player 3 and a single-proposal equilibrium $\Longrightarrow$ This requires the following rank matrix:

$$
\begin{aligned}
A=\left(\begin{array}{lll}
a_{11} & a_{12} & a_{13} \\
a_{12}+\frac{1}{4}-2 \epsilon & \frac{3}{4}-a_{12}+2 \epsilon & 0 \\
0 & \mu_{2} & 1-\mu_{2}
\end{array}\right) & \longrightarrow\left(\begin{array}{lll}
2 & 3 & 2 \\
3 & 1 & 1 \\
1 & 2^{*} & 3
\end{array}\right) \\
D_{1} & =\left(\begin{array}{lll}
0.2 & 0.7 & 0.1
\end{array}\right) \\
D_{2}^{\mathcal{B}_{2}} & =\left(\begin{array}{lll}
0.95-2 \epsilon & 0.05-2 \epsilon & 0.0
\end{array}\right) \\
D_{3} & =\left(\begin{array}{lll}
0.00 & 0.375+\epsilon & 0.625-\epsilon
\end{array}\right)
\end{aligned}
$$

This can be satisfied if the constraints of $D_{3}^{\underline{\mu}}$ hold ( $a_{22}$ has to be non-negative). The feasible set $\mathcal{S}_{\mathcal{B}_{4}}$ is then determined by

$$
\begin{aligned}
& \text { 1. } \mu_{2}<\mu_{1} \Longrightarrow a_{12}>\frac{1}{2}-a_{11} \\
& \text { 2. } 2 a_{11}<a_{21} \Longrightarrow a_{12}>2 a_{11}-\frac{1}{4} \\
& \text { 3. } \quad a_{23} \leq a_{13} \Longrightarrow a_{12} \leq 1-a_{11} \\
& \text { 4. } 1-\mu_{2}+a_{23}>2 a_{13} \Longrightarrow a_{12}>\frac{11}{16}-a_{11} \\
& \text { 5. } \quad a_{22} \geq 0 \Longrightarrow a_{12} \leq \frac{3}{4}
\end{aligned}
$$

After comparison of the inequalities, only 2., 4. and 5. remain.

$$
\begin{aligned}
\Longrightarrow \mathcal{S}_{\mathcal{B}_{4}}= & \left\{\left[0, \frac{3}{16}\right] \times\left(\frac{11}{16}-a_{11}, \frac{3}{4}\right] \cup\left(\frac{3}{16}, \frac{1}{4}\right] \times\left[\frac{1}{2}, \frac{3}{4}\right] \cup\right. \\
& \left.\left(\frac{1}{4}, \frac{3}{8}\right] \times\left[\frac{1}{2}, 1-a_{11}\right] \cup\left[\frac{3}{8}, \frac{5}{12}\right] \times\left(2 a_{11}-\frac{1}{4}, 1-a_{11}\right]\right\}
\end{aligned}
$$

(See Figure 2)

Minimum payoff for Player 2:

$$
\pi_{2}^{\min }\left(\mathcal{B}_{4}\right)>\frac{3}{8}
$$


$\left.\mathcal{B}_{5}\right)$ Suppose $D_{2}=\left(\frac{5}{8}+4 \epsilon, \frac{3}{8}-4 \epsilon, 0\right)$ and $D_{3}=\left(0, \mu_{2}, 1-\mu_{2}\right)$ is the best reaction for Player 3 and implies the correlated equilibrium $C_{13} \Longrightarrow$ This requires the following rank matrix:

$$
\begin{aligned}
& A=\left(\begin{array}{lll}
a_{11} & a_{12} & a_{13} \\
\frac{5}{8}+4 \epsilon & \frac{3}{8}-4 \epsilon & 0 \\
0 & \mu_{2} & 1-\mu_{2}
\end{array}\right) \longrightarrow\left(\begin{array}{lll}
2 & 3 & 2^{*} \\
3 & 1 & 1 \\
1 & 2^{*} & 3
\end{array}\right) \\
& \longrightarrow D_{1}=\left(\begin{array}{llc}
0.05 & 0.61 & 0.34
\end{array}\right) \\
& D_{2}^{\mathcal{B}_{2}}=\left(\begin{array}{lll}
0.625-4 \epsilon & 0.375-4 \epsilon & 0.0
\end{array}\right) \\
& D_{3}=\left(\begin{array}{lll}
0.00 & 0.4925-2 \epsilon & 0.5075+2 \epsilon
\end{array}\right)
\end{aligned}
$$

This can be satisfied if the constraint set (ii) of $D_{3}^{\text {cor }}$ holds $\left(a_{12}=\underline{x}\right)$. The feasible set $\mathcal{S}_{\mathcal{B}_{5}}$ is then determined by the following and the payoff is greater than $\frac{3}{8}$

$$
\begin{aligned}
& \text { 1. } 2 a_{22} \geq a_{12} \quad \Longrightarrow a_{12}<\frac{3}{4} \\
& \text { 2. } 2 a_{11}<a_{21} \quad \Longrightarrow a_{11} \leq \frac{5}{16} \\
& \text { 3. } \mu_{1}+2 a_{22}-a_{12} \leq a_{12} \quad \Longrightarrow a_{12} \geq \frac{17}{32}+\frac{1}{4} a_{11} \\
& \text { 4. } \mu_{1}+2 a_{22}-a_{12} \leq \mu_{2} \quad \Longrightarrow a_{12} \geq \frac{7}{12}+\frac{1}{3} a_{11} \\
& \text { 5. } \quad a_{23} \leq a_{13} \quad \Longrightarrow a_{12} \leq 1-a_{11} \\
& \text { 6. } 1-\left(\mu_{1}+2 a_{22}-a_{12}\right)+a_{23} \leq 2 a_{13} \quad \Longrightarrow a_{12}<\frac{11}{16}-\frac{1}{2} a_{11} \\
& \text { 7. } \quad \frac{1}{2}\left(1-\mu_{2}+a_{13}\right) \leq 1-a_{12} \quad \Longrightarrow a_{12} \geq \frac{3}{8}+2 a_{11} \\
& \text { 8. } 1-\left(2 a_{22}-a_{12}\right)+a_{23}>2 a_{13} \quad \Longrightarrow a_{12} \geq \frac{7}{12}-\frac{2}{3} a_{11} \\
& \text { 9. } \frac{1}{3}\left(1-\left(2 a_{22}-a_{12}\right)+a_{23}+a_{13}\right)<\frac{1}{2}\left(1-\mu_{2}+a_{13}\right) \Longrightarrow a_{12}<\frac{47}{72}-\frac{2}{9} a_{11} \\
& \text { 10. } \quad a_{12}<a_{21} \quad \Longrightarrow a_{12} \leq \frac{5}{8}
\end{aligned}
$$

After comparison of the inequalities only 4. and 10. are remaining.

$$
\Longrightarrow \mathcal{S}_{\mathcal{B}_{5}}=\left\{\left[0, \frac{1}{8}\right] \times\left(\frac{7}{12}+\frac{1}{3} a_{11}, \frac{5}{8}\right)\right\}
$$

(See Figure 2)

Minimum payoff for Player 2:

$$
\pi_{2}^{\min }\left(\mathcal{B}_{5}\right) \geq \frac{51}{96}-\epsilon
$$

$\left.\mathcal{B}_{6}\right)$ Suppose $D_{2}=\left(a_{12}+4 \epsilon, 1-\left(a_{12}+4 \epsilon\right), 0\right)$ and $D_{3}=\left(0, \mu_{2}, 1-\mu_{2}\right)$ is the best reaction for Player 3 and implies the correlated equilibrium $C_{13} \Longrightarrow$ This requires the following rank matrix:

$$
\begin{gathered}
A=\left(\begin{array}{lll}
a_{11} & a_{12} & \begin{array}{l}
a_{13} \\
a_{12}+4 \epsilon
\end{array} \\
0 & 1-\left(a_{12}+4 \epsilon\right) & 0 \\
1-\mu_{2}
\end{array}\right) \longrightarrow\left(\begin{array}{lll}
2 & 3 & 2^{*} \\
3 & 1 & 1 \\
1 & 2^{*} & 3
\end{array}\right) \\
D_{1}=\left(\begin{array}{lll}
0.01 & 0.52 & 0.47
\end{array}\right) \\
D_{2}^{\mathcal{B}_{2}}=\left(\begin{array}{lll}
0.52+4 \epsilon & 0.48-4 \epsilon & 0.0
\end{array}\right) \\
D_{3}=\left(\begin{array}{lll}
0.00 & 0.5-2 \epsilon & 0.5+2 \epsilon
\end{array}\right)
\end{gathered}
$$


This can be satisfied if the constraint set (i) of $D_{3}^{\text {cor }}$ holds (by construction we have $\underline{x}=a_{12}$ ). The feasible set $\mathcal{S}_{\mathcal{B}_{6}}$ is then determined by

$$
\begin{aligned}
& \text { 1. } 2 a_{22} \geq a_{12} \Longrightarrow a_{12}<\frac{2}{3} \\
& \text { 2. } 2 a_{11}<a_{21} \Longrightarrow a_{12} \geq 2 a_{11} \\
& \text { 3. } \mu_{2}<\underline{x} \Longrightarrow a_{12} \geq \frac{1}{2} \\
& \text { 4. } \mu_{1}+2 a_{22}-a_{12} \geq \mu_{2} \Longrightarrow a_{12}<\frac{3}{5}+\frac{a_{11}}{5} \\
& \text { 5. } \quad a_{23}<a_{13} \Longrightarrow a_{12}<1-a_{11} \\
& \text { 6. } 1-\mu_{2}+a_{23} \leq 2 a_{13} \Longrightarrow a_{12}<\frac{3}{4}-a_{11} \\
& \text { 7. } \frac{1}{2}\left(1-\mu_{2}+a_{13}\right)>1-\underline{x} \Longrightarrow a_{12}>\frac{1}{2}+a_{11}
\end{aligned}
$$

After comparison of the inequalities, only 4. and 7. remain.

$$
\Longrightarrow \mathcal{S}_{\mathcal{B}_{6}}=\left\{\left[0, \frac{1}{8}\right] \times\left[\frac{1}{2}+a_{11}, \frac{3}{5}+\frac{1}{5} a_{11}\right)\right\}
$$

(See Figure 2)

Minimum payoff for Player 2:

$$
\pi_{2}^{\min }\left(\mathcal{B}_{6}\right) \geq \frac{1}{2}-\epsilon
$$

$\left.\mathcal{B}_{7}\right)$ Suppose $D_{2}=(1,0,0)$ and $D_{3}=\left(0, \mu_{2}, 1-\mu_{2}\right)$ is the best reaction fir Player 3 and a single-proposal equilibrium $\Longrightarrow$ This requires the following rank matrix:

$$
\begin{gathered}
A=\left(\begin{array}{lll}
a_{11} & a_{12} & a_{13} \\
1 & 0 & 0 \\
0 & \mu_{2} & 1-\mu_{2}
\end{array}\right) \quad \longrightarrow\left(\begin{array}{lll}
2 & 3 & 2 \\
3 & 1 & 1 \\
1 & 2^{*} & 3
\end{array}\right) \\
D_{1}=\left(\begin{array}{lll}
0.01 & 0.86 & 0.03
\end{array}\right) \\
D_{2}^{\mathcal{B}_{2}}=\left(\begin{array}{lll}
1.00 & 0.00 & 0.00
\end{array}\right) \\
D_{3}=\left(\begin{array}{lll}
0.00 & 0.43 & 0.57
\end{array}\right)
\end{gathered}
$$

This can be satisfied if the constraints of $D_{3}^{\mu}$ hold. The feasible set $\mathcal{S}_{\mathcal{B}_{7}}$ is then determined by

$$
\begin{aligned}
& \text { 1. } \quad \mu_{2}<\mu_{1} \Longrightarrow a_{12}<1+a_{11} \\
& \text { 2. } 2 a_{11}<a_{21} \Longrightarrow a_{11}<\frac{1}{2} \\
& \text { 3. } \quad a_{23} \leq a_{13} \Longrightarrow a_{12} \leq 1-a_{11} \\
& \text { 4. } 1-\mu_{2}+a_{23}>2 a_{13} \Longrightarrow a_{12}>\frac{2}{3}-\frac{4}{3} a_{11} \\
& 5 . \\
& \mu_{2}>\frac{3}{8} \Longrightarrow a_{12}>\frac{3}{4}
\end{aligned}
$$

After comparison of the inequalities only 5. remains.

$$
\Longrightarrow \mathcal{S}_{\mathcal{B}_{7}}=\left\{\left[0, \frac{1}{4}\right] \times\left(\frac{3}{4}, 1-a_{11}\right]\right\}
$$

(See Figure 2)

Minimum payoff for Player 2:

$$
\pi_{2}^{\min }\left(\mathcal{B}_{7}\right)>\frac{3}{8}
$$


Thus we have shown that $\forall D_{1} \in \mathcal{A} \cup \mathcal{B} \exists$ a proposal $D_{2}^{s}$ of Player 2, such that $\rho$ is symmetric and $\pi_{2}>\frac{3}{8}$.

\subsubsection{Proof of Corollary 3}

1. Suppose $D_{1} \in \mathcal{A}_{1} \cup \mathcal{A}_{2} \cup \mathcal{B}_{1} \cup \mathcal{B}_{3} \cup \mathcal{B}_{5}$ and $D_{2}$ is such that $\rho$ is non-symmetric and the best reaction for Player 2, then $\pi_{2} \geq \frac{1}{2}$, because if $D_{2}$ is such that $\rho$ is symmetric Player 2 obtains at least $\pi_{2}=\frac{1}{2}$ (see proof of Proposition 3 ). Further, we have $\pi_{3}>\frac{1}{4}$ from the proof of Proposition 2. Together with the resource constraint we obtain $1=\pi_{1}+\pi_{2}+\pi_{3}>\frac{1}{4}+\frac{1}{2}+\frac{1}{4}=1$

2. Suppose $D_{1} \in(\mathcal{A} \cup \mathcal{B}) \backslash\left\{\mathcal{A}_{1} \cup \mathcal{A}_{2} \cup \mathcal{B}_{1} \cup \mathcal{B}_{3} \cup \mathcal{B}_{5}\right\}$ and $D_{2}$ is such that $\rho$ is nonsymmetric and $\pi_{1} \geq \frac{1}{4}$.

First note that $\pi_{1} \geq \frac{1}{4}$ is only possible if the constraints for $D_{3}^{o}$ or $D_{3}^{L^{\epsilon}}$ hold, because otherwise Proposition 2 implies $\pi_{1}=0$ and $\pi_{2} \geq \frac{3}{8}$, since the minimum payoff with $D_{2}$ such that $\rho$ is symmetric is $\pi_{2}=\frac{3}{8}$.

(a) Suppose $D_{2}$ is such that the constraints of $D_{3}^{o}$ hold $\Longrightarrow a=\left(\begin{array}{ll}x_{1} & x_{2} \\ y_{1} & y_{2}\end{array}\right)$ $\Longrightarrow a_{21}+a_{22} \leq \underline{x}$ and $a_{21}=\pi_{1} \geq \frac{1}{4}, a_{22}=\pi_{2} \geq \frac{3}{8} \Longrightarrow \frac{5}{8} \leq \underline{x} \leq \frac{1}{2}$

(b) Suppose $D_{2}$ is such that the constraints of $D_{3}^{L^{\epsilon}}$ hold $\Longrightarrow a=\left(\begin{array}{ll}x_{1} & x_{2} \\ y_{1} & y_{2}\end{array}\right)$ and $D_{3}=\left(0,2 a_{12}-a_{22}+\epsilon, 1-\left(2 a_{12}-a_{22}+\epsilon\right)\right)$ is the best reaction for Player 3 and $\pi_{1}=\frac{1}{3}\left(a_{11}+a_{21}\right)$ with $\pi_{2}=\frac{1}{3}\left(2 a_{12}-a_{22}+\epsilon+a_{12}+a_{22}\right)=a_{12}+\frac{\epsilon}{3}$.

i. Suppose $D_{2} \in \mathcal{A} \backslash\left(\mathcal{A}_{1} \cup \mathcal{A}_{2} \cup \mathcal{A}_{4}\right) \Longrightarrow a_{12} \leq \frac{1}{4} \Longrightarrow$ $\pi_{2}=\frac{1}{4}+\frac{\epsilon}{3}<\frac{3}{8}\left(\epsilon<\frac{3}{8}\right)$

ii. Suppose $D_{2} \in \mathcal{A}_{4} \Longrightarrow \pi_{1}=\frac{1}{6}$

iii. Suppose $D_{2} \in \mathcal{B} \backslash\left(\mathcal{B}_{1} \cup \mathcal{B}_{3} \cup \mathcal{B}_{5}\right) \Longrightarrow a_{11} \leq \frac{3}{8}$ and $2 a_{21}<a_{11}$ (constraints of $\left.D_{3}^{L^{\epsilon}}\right) \Longrightarrow \pi_{1}<\frac{1}{3}\left(\frac{3}{8}+\frac{3}{16}\right)=\frac{3}{16}$

(c) Suppose $D_{2}$ is such that the constraints of $D_{3}^{L^{\epsilon}}$ hold $\Longrightarrow a=\left(\begin{array}{ll}x_{1} & x_{2} \\ y_{1} & y_{2}\end{array}\right)$ and $D_{3}=\left(2 a_{11}-a_{21}+\epsilon, 0,1-\left(2 a_{11}-a_{21}+\epsilon\right)\right)$ with $\pi_{2}=\frac{1}{3}\left(a_{12}+a_{22}\right)$

i. Suppose $D_{2} \in \mathcal{A} \backslash\left(\mathcal{A}_{1} \cup \mathcal{A}_{2} \cup \mathcal{A}_{4}\right) \Longrightarrow a_{12} \leq \frac{1}{6}$ and $\pi_{2} \leq \frac{1}{9}$ since $a_{22} \leq a_{12}$

ii. Suppose $D_{2} \in \mathcal{A}_{4} \Longrightarrow \pi_{2} \leq \frac{1}{3}<\pi_{2}\left(\mathcal{A}_{4}\right)=\frac{1}{2}-\epsilon$ 
iii. Suppose $D_{2} \in \mathcal{B} \backslash\left(\mathcal{B}_{1} \cup \mathcal{B}_{3} \cup \mathcal{B}_{5}\right) \Longrightarrow a_{12} \leq \frac{3}{4}$ and $\pi_{2}<\frac{3}{8}$ since $2 a_{22}<a_{12}$.

\subsubsection{Proof of Proposition 4 and Corollary 4}

Suppose $D_{1} \in \mathcal{C}$

$\left.\mathcal{C}_{1}\right) a_{11}+a_{12} \leq \frac{1}{2}$ and $D_{2}=\left(1-a_{22}, a_{22}, 0\right)$ with $1-\left(2 a_{22}-a_{12}+\epsilon\right)=\frac{1}{2}\left(1-a_{11}-\right.$ $\left.a_{12}\right) \Longrightarrow a_{22}=\frac{1}{4}\left(1+3 a_{12}+a_{11}-2 \epsilon\right)$ and $D_{3}=\left(0,2 a_{22}-a_{12}+\epsilon^{\prime}, 1-\left(2 a_{22}-a_{12}+\epsilon^{\prime}\right)\right)$ is the best reaction for Player 3 and a single-proposal equilibrium. This requires the following rank matrix:

$$
\begin{aligned}
& A=\left(\begin{array}{lll}
a_{11} & a_{12} & a_{13} \\
1-a_{22} & a_{22} & 0 \\
0 & 2 a_{22}-a_{12}+\epsilon^{\prime} & 1-\left(2 a_{22}-a_{12}+\epsilon^{\prime}\right)
\end{array}\right) \quad \longrightarrow \quad\left(\begin{array}{lll}
2 & 1 & 3 \\
3 & 2 & 1 \\
1 & 3 & 2^{*}
\end{array}\right) \\
& D_{1}=\left(\begin{array}{lll}
0.10 & 0.10 & 0.8
\end{array}\right) \\
& D_{2}^{\mathcal{C}_{1}}=\left(\begin{array}{lll}
0.65+\frac{\epsilon}{2} & 0.35-\frac{\epsilon}{2} & 0.0
\end{array}\right. \\
& D_{3}=\left(\begin{array}{lll}
0.00 & 0.6+\epsilon+\epsilon^{\prime} & 0.4-\left(\epsilon+\epsilon^{\prime}\right)
\end{array}\right)
\end{aligned}
$$

This can be satisfied if the constraint set (i) of $D_{3}^{3}$ hold with $a_{22}=\underline{x}$. The feasible set $\mathcal{S}_{\mathcal{C}_{1}}$ is then determined by

$$
\begin{aligned}
& \text { 1. } a_{11}+a_{12} \leq a_{22} \quad \Longrightarrow a_{12}<1-3 a_{11} \\
& \text { 2. } 2 a_{11}<a_{21} \quad \Longrightarrow a_{12} \leq 1-3 a_{11} \\
& \text { 3. } 2 a_{12}<a_{22} \quad \Longrightarrow a_{12}<\frac{1}{5}+\frac{1}{5} a_{11} \\
& \text { 4. } 2 a_{22}-a_{12}<1 \quad \Longrightarrow a_{12} \leq 1-a_{11} \\
& \text { 5. } 1-\left(2 a_{22}-a_{12}+\epsilon^{\prime}\right)>\frac{1}{2}\left(a_{13}+a_{23}\right) \Longrightarrow \epsilon^{\prime}<\epsilon \\
& \text { 6. } \quad a_{22} \leq a_{21} \quad \Longrightarrow a_{12} \leq \frac{1}{3}-\frac{1}{3} a_{11} \\
& \text { 7. } 2 a_{21}-a_{11}>2 a_{22}-a_{12} \Longrightarrow a_{12} \leq \frac{1}{2}-a_{11}
\end{aligned}
$$

After comparison of the inequalities, only 1. and 3. remain.

$$
\Longrightarrow \mathcal{S}_{\mathcal{C}_{1}}=\left\{\left(0, \frac{1}{4}\right] \times\left[0, \frac{1}{5}+\frac{1}{5} a_{11}\right) \cup\left[\frac{1}{4}, \frac{1}{3}\right) \times\left[0,1-3 a_{11}\right)\right\}
$$

(See Figure 3)

Minimum payoff for Player 2:

$$
\pi_{2}^{\min }\left(\mathcal{C}_{1}\right)>\frac{1}{2}
$$


$\left.\mathcal{C}_{2}\right) D_{2}=\left(1-a_{22}, a_{22}, 0\right)$ with $2 a_{21}-a_{11}=1 \Longrightarrow a_{21}=\frac{1}{2}\left(1+a_{11}\right)$ and $a_{22}=\frac{1}{2}\left(1-a_{11}\right)$. $D_{3}=\left(0,2 a_{22}-a_{12}+\epsilon, 1-\left(2 a_{22}-a_{12}+\epsilon\right)\right)$ is the best reaction for Player 3 and a single-proposal equilibrium. This requires the following rank matrix:

$$
\begin{aligned}
& A=\left(\begin{array}{lll}
a_{11} & a_{12} & a_{13} \\
1-a_{22} & a_{22} & 0 \\
0 & 2 a_{22}-a_{12}+\epsilon & 1-\left(2 a_{22}-a_{12}+\epsilon\right)
\end{array}\right) \quad \longrightarrow \quad\left(\begin{array}{lll}
2 & 1 & 3 \\
3 & 2 & 1 \\
1 & 3 & 2^{*}
\end{array}\right) \\
& D_{1}=\left(\begin{array}{lll}
0.08 & 0.30 & 0.62
\end{array}\right) \\
& D_{2}^{\mathcal{C}_{2}}=\left(\begin{array}{lll}
0.54 & 0.46 & 0.00
\end{array}\right. \\
& D_{3}=\left(\begin{array}{lll}
0.00 & 0.62+\epsilon & 0.38-\epsilon
\end{array}\right)
\end{aligned}
$$

This can be satisfied if the constraint set (ii) of $D_{3}^{3}$ holds with $a_{22}=\underline{x}$. The feasible set $\mathcal{S}_{\mathcal{C}_{2}}$ is then determined by

$$
\begin{aligned}
& \text { 1. } a_{11}+a_{12} \leq a_{22} \quad \Longrightarrow a_{12} \leq \frac{1}{2}-\frac{3}{2} a_{11} \\
& \text { 2. } 2 a_{11}<a_{21} \quad \Longrightarrow a_{11}<\frac{1}{3} \\
& \text { 3. } 2 a_{12} \geq a_{22} \quad \Longrightarrow a_{12} \geq \frac{1}{4}-\frac{1}{4} a_{11} \\
& \text { 4. } 2 a_{22}-a_{12}<1 \quad \Longrightarrow a_{12}>-a_{11} \\
& \text { 5. } \quad 2 a_{21}-a_{11} \geq 1 \quad \Longrightarrow \quad 1 \geq 1 \\
& \text { 6. } 1-\left(2 a_{22}-a_{12}+\epsilon\right) \geq \frac{1}{2}\left(a_{13}+a_{23}\right) \Longrightarrow a_{12}>\frac{1}{3}-a_{11} \\
& \text { 7. } \quad a_{22} \leq a_{21} \quad \Longrightarrow a_{11} \geq 0 \\
& \text { 8. } \quad 2 a_{22}-a_{12} \geq \frac{1}{2} \quad \Longrightarrow a_{12} \leq \frac{1}{2}-a_{11}
\end{aligned}
$$

After comparison of the inequalities, only 1., 3. and 6. remain.

$$
\Longrightarrow \mathcal{S}_{\mathcal{C}_{2}}=\left\{\left[0, \frac{1}{9}\right] \times\left(\frac{1}{3}-a_{11}, \frac{1}{2}-\frac{3}{2} a_{11}\right] \cup\left(\frac{1}{9}, \frac{1}{5}\right] \times\left[\frac{1}{4}-\frac{1}{4} a_{11}, \frac{1}{2}-\frac{3}{2} a_{11}\right]\right\}
$$

(See Figure 3)

Minimum payoff for Player 2:

$$
\pi_{2}^{\min }\left(\mathcal{C}_{2}\right)>\frac{1}{2}
$$

$\left.\mathcal{C}_{3}\right) D_{2}=\left(1-a_{22}, a_{22}, 0\right)$ with $2 a_{22}-a_{12}=\frac{1}{2} \Longrightarrow a_{21}=\frac{1}{4}\left(3-2 a_{12}\right)$ and $a_{22}=$ $\frac{1}{4}\left(1+2 a_{12}\right) . \quad D_{3}=\left(0,2 a_{22}-a_{12}+\epsilon, 1-\left(2 a_{22}-a_{12}+\epsilon\right)\right)$ is the best reaction for Player 3 and a single-proposal equilibrium. This requires the following rank matrix:

$$
\begin{aligned}
& A=\left(\begin{array}{lll}
a_{11} & a_{12} & a_{13} \\
1-a_{22} & a_{22} & 0 \\
0 & \frac{1}{2}+\epsilon & \left.\frac{1}{2}+\epsilon\right)
\end{array}\right) \longrightarrow\left(\begin{array}{lll}
2 & 1 & 3 \\
3 & 2 & 1 \\
1 & 3 & 2^{*}
\end{array}\right) \\
& D_{1}=\left(\begin{array}{lll}
0.02 & 0.24 & 0.74
\end{array}\right) \\
& D_{2}^{\mathcal{C}_{3}}=\left(\begin{array}{lll}
0.63 & 0.37 & 0.0
\end{array}\right. \\
& D_{3}=\left(\begin{array}{lll}
0.00 & 0.5+\epsilon & 0.5-\epsilon
\end{array}\right)
\end{aligned}
$$


This can be satisfied if the constraint set (ii) of $D_{3}^{3}$ holds with $a_{22}=\underline{x}$. The feasible set $\mathcal{S}_{\mathcal{C}_{3}}$ is then determined by

$$
\begin{aligned}
& \text { 1. } a_{11}+a_{12} \leq a_{22} \quad \Longrightarrow a_{12} \leq \frac{1}{2}-2 a_{11} \\
& \text { 2. } 2 a_{11}<a_{21} \quad \Longrightarrow a_{12}<\frac{3}{2}-4 a_{11} \\
& \text { 3. } \quad 2 a_{12} \geq a_{22} \quad \Longrightarrow a_{12} \geq \frac{1}{6} \\
& \text { 4. } 2 a_{22}-a_{12}<1 \quad \Longrightarrow \quad \frac{1}{2}<1 \\
& \text { 5. } \quad 2 a_{21}-a_{11} \geq 1 \quad \Longrightarrow a_{12} \leq \frac{1}{2}-a_{11} \\
& \text { 6. } 1-\left(2 a_{22}-a_{12}+\epsilon\right) \geq \frac{1}{2}\left(a_{13}+a_{23}\right) \Longrightarrow a_{12}>-a_{11} \\
& \text { 7. } \quad a_{22} \leq a_{21} \quad \Longrightarrow a_{12} \leq \frac{1}{2}
\end{aligned}
$$

After comparison of the inequalities, only 1. and 3. remain.

(See Figure 3)

$$
\Longrightarrow \mathcal{S}_{\mathcal{C}_{3}}=\left\{\left[0, \frac{1}{6}\right] \times\left[\frac{1}{6}, \frac{1}{2}-2 a_{11}\right]\right\}
$$

Minimum payoff for Player 2:

$$
\pi_{2}^{\min }\left(\mathcal{C}_{3}\right)>\frac{1}{2}
$$

$\left.\mathcal{C}_{4}\right) D_{2}=\left(1-a_{22}, a_{22}, 0\right)$ with $2 a_{22}-a_{12}+\epsilon=2 a_{21}-a_{11} \Longrightarrow a_{21}=\frac{1}{4}\left(2+a_{11}-a_{21}+\epsilon\right)$ and $a_{21}=\frac{1}{4}\left(2-a_{11}+a_{21}-\epsilon\right) . \quad D_{3}=\left(0,2 a_{22}-a_{12}+\epsilon^{\prime}, 1-\left(2 a_{22}-a_{12}+\epsilon^{\prime}\right)\right)$ is the reaction for Player 3 and a single-proposal equilibrium. This requires the following rank matrix:

$$
\begin{aligned}
& A=\left(\begin{array}{lll}
a_{11} & a_{12} & a_{13} \\
1-a_{22} & a_{22} & 0 \\
0 & 2 a_{22}-a_{12}+\epsilon^{\prime} & 1-\left(2 a_{22}-a_{12}+\epsilon^{\prime}\right)
\end{array}\right) \quad \longrightarrow \quad\left(\begin{array}{lll}
2 & 1 & 3 \\
3 & 2 & 1 \\
1 & 3 & 2
\end{array}\right) \\
& \begin{array}{lll}
D_{1} & =\left(\begin{array}{lll}
0.06 & 0.10 & 0.84
\end{array}\right)
\end{array} \\
& D_{2}^{\mathcal{C}_{4}}=\left(\begin{array}{lll}
0.49+\epsilon & 0.51-\epsilon & 0.0
\end{array}\right.
\end{aligned}
$$

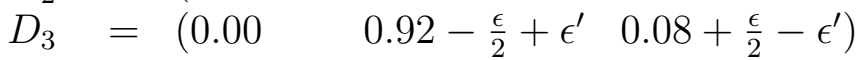

This can be satisfied if the constraint set (i) of $D_{3}^{T_{\epsilon}}$ holds with $a_{21}=\underline{x}$. The feasible set $\mathcal{S}_{\mathcal{C}_{4}}$ is then determined by

$$
\begin{aligned}
& \text { 1. } a_{11}+a_{12} \leq a_{21} \quad \Longrightarrow a_{12} \leq \frac{2}{5}-\frac{3}{5} a_{11} \\
& \text { 2. } 2 a_{11}<a_{21} \quad \Longrightarrow a_{12} \leq 2-7 a_{11} \\
& \text { 3. } 2 a_{12}<a_{22} \quad \Longrightarrow a_{12}<\frac{2}{7}-\frac{1}{7} a_{11} \\
& \text { 4. } 2 a_{22}-a_{12}<1 \quad \Longrightarrow a_{12} \geq-a_{11} \\
& \text { 5. } 1-\left(2 a_{22}-a_{12}+\epsilon^{\prime}\right)<\frac{1}{2}\left(a_{13}+a_{23}\right) \Longrightarrow a_{12}<\frac{1}{2}-a_{11} \\
& \text { 6. } \quad a_{21} \leq a_{22} \quad \Longrightarrow a_{12}>a_{11} \\
& \text { 7. } 2 a_{21}-a_{11}>2 a_{22}-a_{12} \Longrightarrow \epsilon>0
\end{aligned}
$$

After comparison of the inequalities, only 3. and 6. remain. 
(See Figure 3)

$$
\Longrightarrow \mathcal{S}_{\mathcal{C}_{4}}=\left\{\left[0, \frac{1}{4}\right] \times\left(a_{11}, \frac{2}{7}-\frac{1}{7} a_{11}\right)\right\}
$$

Minimum payoff for Player 2:

$$
\pi_{2}^{\min }\left(\mathcal{C}_{4}\right)>\frac{1}{2}-\epsilon
$$

$\mathcal{C}_{5}$ ) In the remaining part $\mathcal{S}_{\mathcal{C}_{5}}=\left\{\mathcal{C} \backslash \bigcup_{k=1}^{4} \mathcal{S}_{\mathcal{C}_{k}}\right\}$ (see Figure 3), we show that, given $\left(a_{11}, a_{12}\right) \in \mathcal{S}_{\mathcal{C}_{5}}$ the share of Player 1 is $\pi_{1}=0$ if Player 2's best proposal implies $\rho$ to be non-symmetric or the share of Player 2 is $\pi_{2}>\frac{3}{8}$ if Player 2's best proposal implies $\rho$ to be symmetric.

In order to prove this, we divide $\mathcal{S}_{\mathcal{C}_{5}}$ into three subsets:

$$
\begin{aligned}
& \mathcal{S}_{\mathcal{C}_{5}}^{1}=\left\{\left(a_{11}, a_{12}\right) \mid a_{11}+a_{12}>\frac{1}{2}, a_{11}<\frac{1}{2}, a_{12}<\frac{1}{2}\right\} \\
& \mathcal{S}_{\mathcal{C}_{5}}^{2}=\left\{\left(a_{11}, a_{12}\right) \mid a_{11}+a_{12} \leq \frac{1}{2}, a_{12} \geq \frac{2}{7}-\frac{1}{7} a_{11}, a_{12}>\frac{1}{2}-\frac{3}{2} a_{11}\right\} \\
& \mathcal{S}_{\mathcal{C}_{5}}^{3}=\left\{\left(a_{11}, a_{12}\right) \mid a_{11}+a_{12} \leq \frac{1}{2}, a_{12} \geq 1-3 a_{11}, a_{11} \geq 0\right\}
\end{aligned}
$$

(a) Given $\left(a_{11}, a_{12}\right) \in \mathcal{S}_{\mathcal{C}_{5}}^{1} \Longrightarrow$ with $D_{2}=\left(\frac{1}{2}+\epsilon, \frac{1}{2}-\epsilon, 0\right)$, the inequalities of $D_{\frac{x^{\epsilon}}{3}}$ hold and $D_{3}=\left(0, \frac{1}{2}-\epsilon+\epsilon^{\prime}, \frac{1}{2}+\epsilon-\epsilon^{\prime}\right)$ is the best proposal for Player 3 with $D_{3}$ being a proposal equilibrium including $\pi_{1}=0$ and $\pi_{2}=\frac{1}{2}-\epsilon+\epsilon^{\prime}$.

i. Suppose Player 2 constructs his proposal $D_{2}$ in such a way that $\rho$ is non-symmetric and $\pi_{1}>0$, then the inequalities of $D_{3}^{L^{\epsilon}}$ or $D_{3}^{o}$ must hold $\left(a_{11}=y_{1}, a_{12}=y_{2}\right)$. But $y_{1}+y_{2} \leq \underline{x}$ cannot hold since $\underline{x} \leq \frac{1}{2}$.

ii. Suppose Player 2 constructs his proposal $D_{2}$ in such a way that $\rho$ is symmetric and $\pi_{2} \leq \frac{3}{8}$, then this cannot be his best proposal as proposing $D_{2}=\left(\frac{1}{2}+\epsilon, \frac{1}{2}-\epsilon, 0\right)$ with non-symmetric $\rho$ and $\epsilon<\frac{1}{8}$ ensures him a share of $\pi_{2}>\frac{3}{8}$.

(b) Given $\left(a_{11}, a_{12}\right) \in \mathcal{S}_{\mathcal{C}_{5}}^{2} \cup \mathcal{S}_{\mathcal{C}_{5}}^{3} \Longrightarrow$ with $D_{2}=\left(1-\left(a_{11}+a_{12}-\epsilon\right), a_{11}+a_{12}-\epsilon, 0\right)$ the inequalities of $D_{3}^{x^{\epsilon}}$ hold and $D_{3}=\left(0, a_{11}+a_{12}-\epsilon+\epsilon^{\prime}, 1-\left(a_{11}+a_{12}-\right.\right.$ $\left.\epsilon+\epsilon^{\prime}\right)$ is the best proposal for Player 3 with $D_{3}$ being a proposal equilibrium including $\pi_{1}=0$ and $\pi_{2}=a_{12}+a_{11}-\epsilon+\epsilon^{\prime}$.

i. Suppose Player 2 constructs his proposal $D_{2}$ in such a way that $\rho$ is nonsymmetric, $\pi_{1}>0$, and the inequalities of $D_{3}^{o}$ hold, with $\pi_{2}=a_{12}$. This cannot be the best reaction for Player 2 as $D_{2}=\left(1-\left(a_{11}+a_{12}-\epsilon\right), a_{11}+\right.$ $\left.a_{12}-\epsilon, 0\right)$ with $\epsilon<a_{11}$ ensures him a share of $\pi_{2}=a_{11}+a_{12}-\epsilon+\epsilon^{\prime}>a_{12}$.

ii. Suppose Player 2 constructs his proposal $D_{2}$ in such a way that $\rho$ is non-symmetric, $\pi_{1}>0$ and the inequalities of $D_{3}^{L^{\epsilon}}$ hold with $D_{3}=$ $\left(2 a_{21}-a_{11}+\epsilon, 0,1-\left(2 a_{21}-a_{22}+\epsilon\right)\right)$ is the best proposal for Player $3\left(a_{11}=\right.$ $\left.y_{1}, a_{12}=y_{2}\right) \Longrightarrow$ This cannot be the best reaction for Player 2 because his share $\pi_{2}=\frac{1}{3}\left(x_{2}+y_{2}\right)$ must exceed his share $\pi_{2}=y_{1}+y_{2}+\epsilon-\epsilon^{\prime}$ generated by $D_{2}=\left(0, \frac{1}{2}-\epsilon+\epsilon^{\prime}, \frac{1}{2}+\epsilon-\epsilon^{\prime}\right)$. But

$$
\frac{1}{3}\left(x_{2}+y_{2}\right) \geq y_{1}+y_{2} \quad \stackrel{y_{2} \geq 1-3 y_{1} \geq \frac{1}{2}-\frac{3}{2} y_{1}}{\Longrightarrow} \quad x_{2}=1 \stackrel{x_{1} \leqq 1-x_{2}}{\Longrightarrow} x_{1}=0=\underline{x}
$$

since $a_{12} \geq \frac{1}{4}$ and $a_{11}+a_{12} \leq \underline{x}$ 
iii. Suppose Player 2 constructs his proposal $D_{2}$ in such a way that $\rho$ is symmetric and $\pi_{2} \leq \frac{3}{8} \Longrightarrow$

- $a_{11}+a_{12} \leq \frac{3}{8}$ and

- $D_{3} \in\left\{D_{3}^{\text {cor }}, D_{3}^{L}\right\}$ must hold.

Otherwise Player 2 can obtain a share of $\pi_{2}=a_{11}+a_{12}-\epsilon+\epsilon^{\prime}>$ $\frac{3}{8} \geq a_{11}>a_{12}$ by choosing $\epsilon<a_{11}+a_{12}-\frac{3}{8}$ and proposing $D_{2}=$ $\left(1-\left(a_{11}+a_{12}-\epsilon\right), a_{11}+a_{12}-\epsilon, 0\right)$ with non-symmetric $\rho$. This also implies $D_{3} \in\left\{D_{3}^{\text {cor }}, D_{3}^{L}\right\}$ following from Corollary 5 , because if $\left(a_{11}, a_{12}\right) \in$ $\left\{\left(\mathcal{S}_{\mathcal{C}_{5}}^{2} \cup \mathcal{S}_{\mathcal{C}_{5}}^{3}\right) \cap\left[0, \frac{3}{8}\right] \times\left[0, \frac{3}{8}-a_{11}\right]\right\}=\left\{\left[\frac{5}{16}, \frac{1}{3}\right] \times\left[1-3 a_{11}, \frac{3}{8}-a_{11}\right] \cup\left[\frac{1}{3}, \frac{3}{8}\right] \times\right.$ $\left.\left[0, \frac{3}{8}-a_{11}\right]\right\}$ and given symmetric $\rho$, we have $\underline{x} \leq a_{11} \leq \frac{3}{8} \vee \underline{x} \leq a_{12} \leq \frac{1}{16}$.

$\alpha$. Suppose Player 2 constructs his proposal $D_{2}$ in such a way that $\rho$ is symmetric and $D_{3}=D_{3}^{\text {cor }}$ is the best proposal for Player 3 leading to a correlated equilibrium $C_{23} \Longrightarrow$

This cannot be the best reaction for Player 2 because his share $\pi_{2}\left(D_{3}^{\text {cor }}\right)=\frac{1}{2}\left(\mu_{2}+x_{2}\right)=\frac{1}{2}\left(\frac{1}{2}\left(a_{22}+a_{12}\right)+a_{22}\right)=\frac{3}{4} a_{22}+\frac{1}{4} a_{12}$ must exceed $\pi_{2}\left(D_{3}^{\underline{x}^{\epsilon}}\right)=a_{11}+a_{12}-\epsilon+\epsilon^{\prime}$, which implies

$$
\begin{gathered}
\frac{3}{4} a_{22}+\frac{1}{4} a_{12} \underset{\substack{a_{11} \geq \frac{5}{16} \\
a_{12} \geq 0}}{\geq} a_{11}+a_{12} \Longrightarrow a_{22} \geq \frac{4}{3} a_{11}+a_{12} \\
\geq \quad \frac{5}{12}
\end{gathered}
$$

, But this is a contradiction of

$$
\hat{R}\left(D_{3}^{\text {cor }}\right)=\left(\begin{array}{lll}
3 & 1 & 1 \\
2 & 3 & 2^{*} \\
1 & 2^{*} & 3
\end{array}\right)
$$

which implies

$$
a_{23} \stackrel{a_{11}+a_{12} \leq \frac{3}{8}}{\geq} \frac{5}{8} \Longrightarrow a_{22} \leq \frac{3}{8}
$$

$\beta$. Suppose Player 2 constructs his proposal $D_{2}$ in such a way that $\rho$ is symmetric and $D_{3}=D_{3}^{\text {cor }}$ is the best proposal for Player 3 leading to a correlated equilibrium $C_{13} \Longrightarrow$

This cannot be the best reaction for Player 2 since his share is given by $\pi_{2}=\frac{1}{2}\left(a_{12}+a_{32}\right) \leq a_{12}$ because $\Phi\left(a_{32}\right) \leq 2$.

$\gamma$. Suppose Player 2 constructs his proposal $D_{2}$ in such a way that $\rho$ is symmetric and $D_{3}=D_{3}^{L}$ is the best proposal for Player 3 leading to a selection of the proposal by drawing lots $\Longrightarrow$

This cannot be the best reaction for Player 2 because if $a_{32}=2 a_{12}-$ $a_{22}+\epsilon$ we have $\pi_{2}\left(D_{3}^{L}\right)=\frac{1}{3}\left(a_{12}+a_{22}+a_{32}\right)=a_{12}+\frac{\epsilon}{3}<\pi_{2}\left(D_{3}^{x^{\epsilon}}\right)$ and if $a_{32}=0, \pi_{2}\left(D_{3}^{L}\right)>\pi_{2}\left(D_{3}^{\underline{x}^{\epsilon}}\right)$ would imply

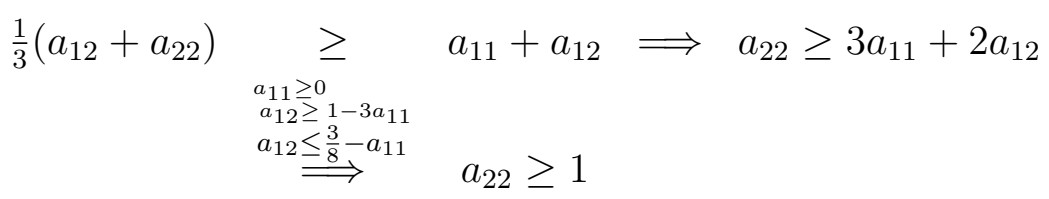


But then we have $a_{23}=0, a_{13} \geq \frac{5}{8}$, and $a_{33} \leq 1$, which cannot lead to a decision by drawing lots with $\Phi\left(a_{33}\right)=3$.

iv. Suppose $\left(a_{11}, a_{12}\right) \in \mathcal{S}_{\mathcal{C}_{5}}^{2}$ and Player 2 constructs his proposal $D_{2}$ in such a way that $\rho$ is non-symmetric, $\pi_{1}>0$, and the inequalities of $D_{3}^{L^{\epsilon}}(i)$ hold with $D_{3}=\left(0,2 a_{22}-a_{12}+\epsilon, 1-\left(2 a_{22}-a_{12}+\epsilon\right)\right)$ is the best proposal for Player $3\left(a_{11}=y_{1}, a_{12}=y_{2}\right) \Longrightarrow$

$$
\begin{array}{cccc}
3 y_{2} & \substack{2 y_{2}<x_{2} \\
x_{1} \leq 1-x_{2}} & 2 x_{2}-y_{2} & 2 x_{2}-y_{2}<2 x_{1}-y_{1} \\
\leq & 2\left(1-x_{2}\right)-y_{1} & \stackrel{2 y_{2}<x_{2}}{<} & 2 x_{1}-y_{1}
\end{array} \quad \Longrightarrow y_{2}<\frac{2}{7}-\frac{1}{7} y_{1}
$$

since $a_{12} \geq \frac{2}{7}-\frac{1}{7} a_{11}$ must hold in $\mathcal{S}_{\mathcal{C}_{5}}^{2}$.

v. Suppose $\left(a_{11}, a_{12}\right) \in \mathcal{S}_{\mathcal{C}_{5}}^{2}$ and Player 2 constructs his proposal $D_{2}$ in such a way that $\rho$ is non-symmetric, $\pi_{1}>0$, and the inequalities of $D_{3}^{L^{\epsilon}}(i i)$ hold with $D_{3}=\left(0,2 a_{22}-a_{12}+\epsilon, 1-\left(2 a_{22}-a_{12}+\epsilon\right)\right)$ is the best proposal for Player $3\left(a_{11}=y_{1}, a_{12}=y_{2}\right) \Longrightarrow$

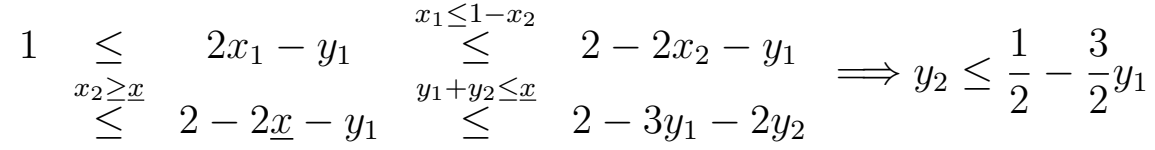

since $a_{12} \leq \frac{1}{2}-\frac{3}{2} a_{11}$ must hold in $\mathcal{S}_{\mathcal{C}_{5}}^{2}$.

vi. Suppose $\left(a_{11}, a_{12}\right) \in \mathcal{S}_{\mathcal{C}_{5}}^{3}$ and Player 2 constructs his proposal $D_{2}$ in such a way that $\rho$ is non-symmetric, $\pi_{1}>0$, and the inequalities of $D_{3}^{L^{\epsilon}}$ hold with $D_{3}=\left(0,2 a_{22}-a_{12}+\epsilon, 1-\left(2 a_{22}-a_{12}+\epsilon\right)\right)$ is the best proposal for Player $3\left(a_{11}=y_{1}, a_{12}=y_{2}\right) \Longrightarrow$

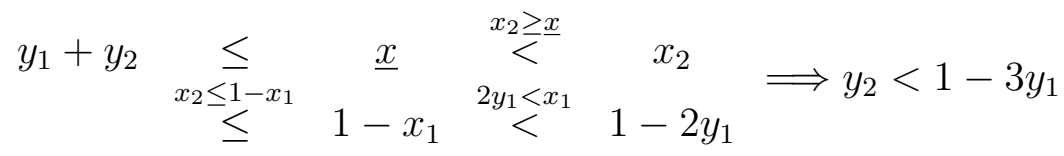

since $a_{12} \geq 1-3 a_{11}$ must hold in $\mathcal{S}_{\mathcal{C}_{5}}^{3}$.

Thus we have proved Proposition 4 and Corollary 4 .

\subsubsection{Proof of Proposition 5}

In the following, we only give binding constraints.

If $a=\left(\begin{array}{cc}0 & 1 \\ 1 & 0\end{array}\right) \Longrightarrow \pi_{2}=\frac{1}{4}$ is already shown in Corollary 1 .

1. Suppose $a=\left(\begin{array}{cc}0 & 1 \\ 0 & a_{22}\end{array}\right) \Longrightarrow D_{3}=(\epsilon, 0,1-\epsilon)$ is the best reaction for Player 3 with $D_{3}$ being a single-proposal equilibrium $\Longrightarrow \pi_{2}=0$ 
The solution set is given by

(See Figure 4)

$$
\mathcal{S}_{1}=\{[0,0] \times[0,1]\}
$$

2. Suppose $a=\left(\begin{array}{cc}0 & 1 \\ a_{21} & a_{22}\end{array}\right)$ and

$$
\begin{aligned}
\text { 1. } a_{22} & <\frac{1}{2} \\
\text { 2. } 1-\mu_{1} & >2 a_{23}
\end{aligned} \Longrightarrow a_{22}>\frac{1}{2}-\frac{3}{4} a_{21}
$$

then the constraints of $D^{\underline{\mu}}$ hold and $D^{\underline{\mu}}=\left(\mu_{1}, 0,1-\mu_{1}\right)$ is the best reaction for Player 3 with $D_{3}^{\underline{\mu}}$ being a single-proposal equilibrium. $\Longrightarrow \pi_{2}=0$

The solution set is given by

$$
\mathcal{S}_{2}=\left\{\begin{array}{l}
{\left[0, \frac{1}{2}\right] \times\left(\frac{1}{2}-\frac{3}{4} a_{21}, \frac{1}{2}\right) \cup} \\
\left(\frac{1}{2}, \frac{2}{3}\right] \times\left(\frac{1}{2}-\frac{3}{4} a_{21}, 1-a_{21}\right] \cup \\
\left.\left(\frac{2}{3}, 1\right) \times\left[0,1-a_{21}\right]\right\}
\end{array}\right.
$$

(See Figure 4)

3. Suppose $a=\left(\begin{array}{cc}0 & 1 \\ a_{21} & a_{22}\end{array}\right)$ and

$$
\begin{aligned}
& \text { 1. } a_{22}<\frac{1}{2}
\end{aligned}
$$

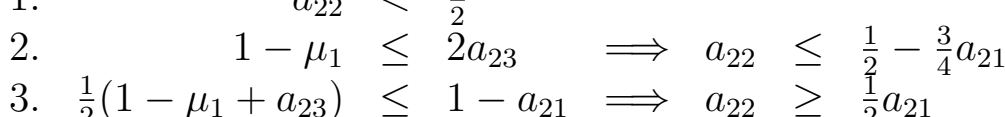

then the constraints (ii) of $D_{3}^{\underline{x}}$ hold and $D^{\underline{x}}=(\underline{x}, 0,1-\underline{x})$ is the best reaction for Player 3 with $D_{3}^{\underline{x}}$ being a single-proposal equilibrium. $\Longrightarrow \pi_{2}=0$

The solution set is given by

(See Figure 4)

$$
\mathcal{S}_{3}=\left\{\left(0, \frac{2}{5}\right] \times\left[\frac{1}{2} a_{21}, \frac{1}{2}-\frac{3}{4} a_{21}\right]\right\}
$$

4. Suppose $a=\left(\begin{array}{cc}0 & 1 \\ a_{21} & a_{22}\end{array}\right)$ and

$$
\text { 1. } \begin{aligned}
1-\mu_{1} & \leq 2 a_{23} \Longrightarrow a_{22} \leq \frac{1}{2}-\frac{3}{4} a_{21} \\
\text { 2. } \frac{1}{2}\left(1-\mu_{1}+a_{23}\right) & >1-a_{21} \Longrightarrow a_{22}<\frac{1}{2} a_{21}
\end{aligned}
$$

then the constraints (a) of $D_{3}^{\text {cor }}$ hold and $D_{3}^{\text {cor }}=\left(\mu_{1}, 0,1-\mu_{1}\right)$ is the best reaction for Player 3 with $C_{23}$ being a correlated equilibrium. $\Longrightarrow \pi_{2}=\frac{1}{2} a_{22}<\frac{1}{10}$ The solution set is given by

$$
\mathcal{S}_{4}=\left\{\left(0, \frac{2}{5}\right] \times\left[0, \frac{1}{2} a_{21}\right) \cup\left(\frac{2}{5}, \frac{2}{3}\right] \times\left[0, \frac{1}{2}-\frac{3}{4} a_{21}\right)\right\}
$$

(See Figure 4) 
5. Suppose $a=\left(\begin{array}{cc}0 & 1 \\ a_{21} & a_{22}\end{array}\right)$ and

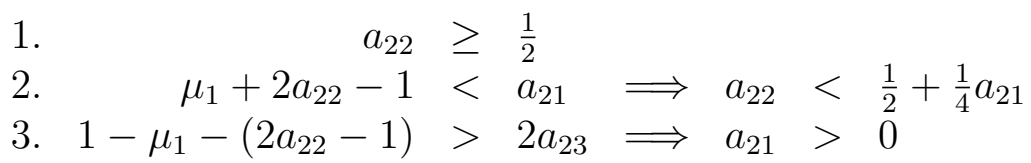

then the constraints (i) of $D_{3}^{\mu_{i}}$ hold and $D_{3}^{\mu_{i}}=\left(\mu_{1}, 2 a_{22}-1+\epsilon, 1-\left(\mu_{1}+2 a_{22}-1+\epsilon\right)\right)$ is the best reaction for Player 3 with $D_{3}^{\mu_{i}}$ being a single-proposal equilibrium. $\Longrightarrow$ $\pi_{2}=2 a_{22}-1+\epsilon<\frac{1}{5}+\epsilon$

The solution set is given by

$$
\mathcal{S}_{5}=\left\{\left(0, \frac{2}{5}\right] \times\left[\frac{1}{2}, \frac{1}{2}+\frac{1}{4} a_{21}\right) \cup\left(\frac{2}{5}, \frac{1}{2}\right] \times\left[\frac{1}{2}, 1-a_{21}\right]\right\}
$$

(See Figure 4)

6. Suppose $a=\left(\begin{array}{cc}0 & 1 \\ a_{21} & a_{22}\end{array}\right)$ and

$$
\text { 1. } \begin{aligned}
a_{21} & >0 \\
\text { 2. } \mu_{1}+2 a_{22}-1 & \geq a_{21} \Longrightarrow a_{22} \geq \frac{1}{2}+\frac{1}{4} a_{21}
\end{aligned}
$$

then the constraints (i) of $D^{\underline{x}}$ hold and $D^{\underline{x}}=(\underline{x}, 0,1-\underline{x})$ is the best reaction for Player 3 with $D^{\frac{x}{3}}$ being a single proposal equilibrium. $\Longrightarrow \pi_{2}=0$

The solution set is given by

$$
\mathcal{S}_{6}=\left\{\left(0, \frac{2}{5}\right] \times\left[\frac{1}{2}+\frac{1}{4} a_{21}, 1-a_{21}\right]\right\}
$$

(See Figure 4)

Altogether we have shown that $D_{2}=(1,0,0)$ is the best reaction for Player 2 given $D_{1}=(0,1,0)$ 


\section{Literature}

Aumann, R., 1974, Subjectivity and Correlation in Randomized Strategies, Journal of Mathematical Economics, 1, 67-96.

Baron, D.P., Ferejohn, J., 1989, Bargaining in legislatures, American Political Science Review, 83, 1181-1206.

Bernholz, P., Breyer, F., 1994, Grundlagen der Politischen Ökonomie, Vol. 2: Ökonomische Theorie der Politik, $3^{\text {rd }}$ edition, Tübingen.

Binmore, K., Rubinstein, A., Wolinsky, A. 1986, The Nash bargaining solution in economic modelling, Rand Journal of Economics 17, 176-188.

Fudenberg, D., Tirole, J., 1992, Game Theory, The MIT Press, Cambridge, Massachusetts.

Gersbach, H., Wehrspohn, U., 2001, "Dividing a cake by majority decisions", mimeo.

Harrington, J.E., Jr. 1986, A noncooperative bargaining game with risk averse players and an uncertain finite horizon, Economics Letter 20, 9-13.

Kihlstrom, R., Roth, A.E., Schmeidler, D. , 1981, Risk aversion and solutions to Nash's bargaining problem, in: O. Moeschlin and D. Pallaschke, eds., Game Theory and Mathematical Economics, North-Holland, Amsterdam.

Krehbiel, K., 1991, Information and Legislative Organization, The University of Michigan Press, Ann Arbor.

Mueller, D.C., 1978, Voting by veto, Journal of Public Economics 10, 57-75.

Myerson, R.B., 1991, Game Theory: Analysis of Conflict, Harvard University Press.

Nielsen, L.T., 1984, Risk sensitivity in bargaining with more than two participants, Journal of Economic Theory 32, 371-376.

Osborne, M.J., 1985, The role of risk aversion in a simple bargaining model, in A.E. Roth, ed., Game-theoretic models of bargaining. Cambridge University Press, Cambridge.

Roth, A.E., 1979, Axiomatic models of bargaining, Lecture Notes in Economics and Mathematical Systems 170, Springer, Berlin.

Roth, A.E., 1985, A note on risk aversion in a perfect equilibrium model of bargaining, Econometrica 53, 207-211.

Roth, A.E., and U.G. Rothblum, 1982, Risk aversion and Nash's solution for bargaining games with risky outcomes, Econometrica 50, 639-648.

Selten, R., 1975, Reexamination of the perfectness concept for equilibrium points in extensive games, International Journal of Game theory 4, 25-55.

Zeuthen, F., 1930, Problems of monopoly and economic warfare, Rutledge, London. 


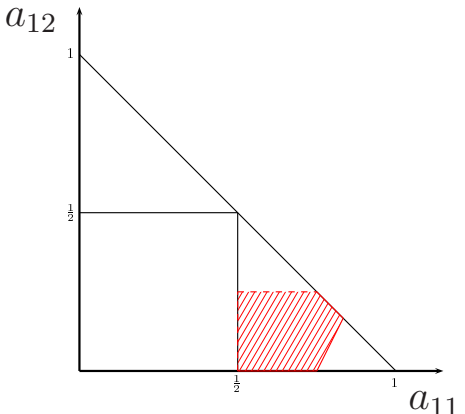

Feasible set $\mathcal{S}_{\mathcal{A}_{1}}$

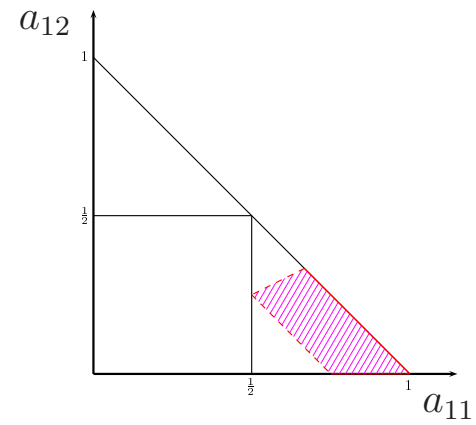

Feasible set $\mathcal{S}_{\mathcal{A}_{3}}$

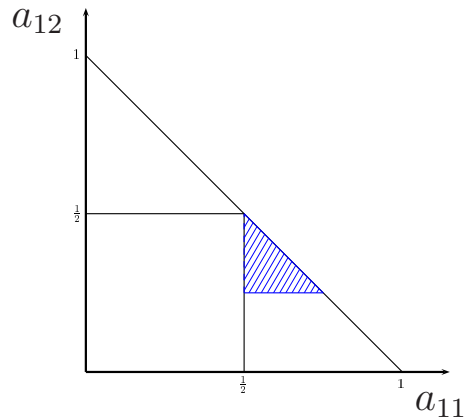

Feasible set $\mathcal{S}_{\mathcal{A}_{2}}$

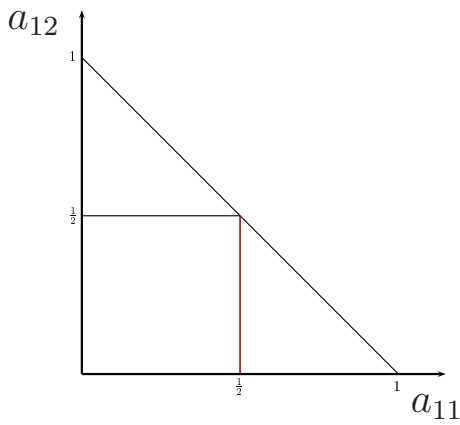

Feasible set $\mathcal{S}_{\mathcal{A}_{4}}$

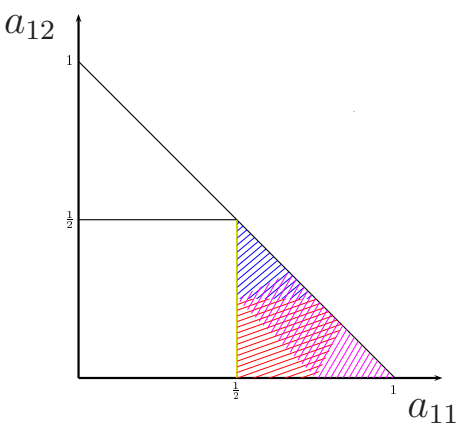

Feasible sets $\mathcal{S}_{\mathcal{A}_{1}}-\mathcal{S}_{\mathcal{A}_{4}}$

Figure 1: Feasible set $\mathcal{S}_{\mathcal{A}}$. 


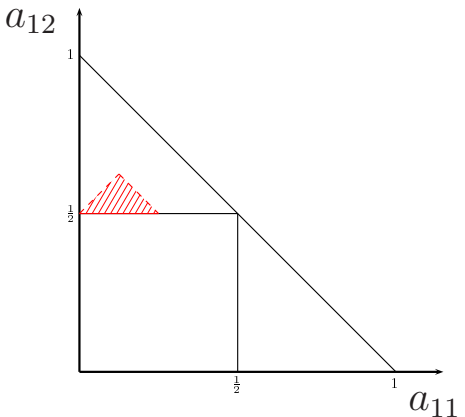

Feasible set $\mathcal{S}_{\mathcal{B}_{1}}$

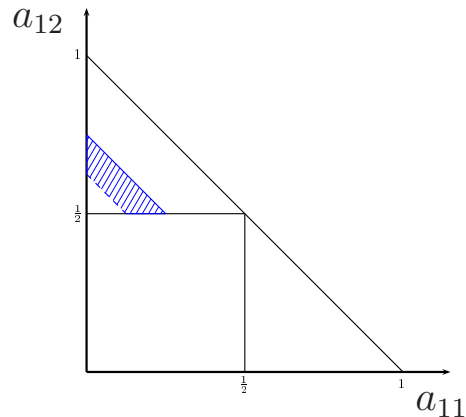

Feasible set $\mathcal{S}_{\mathcal{B}_{2}}$

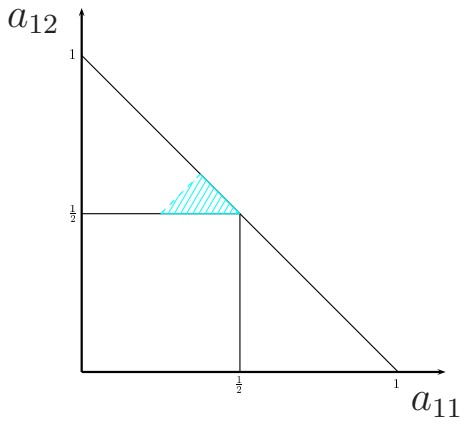

Feasible set $\mathcal{S}_{\mathcal{B}_{3}}$

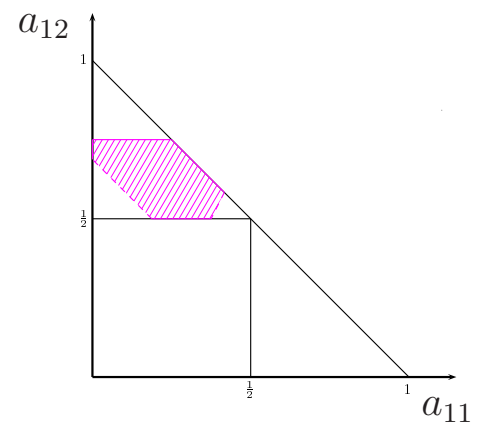

Feasible set $\mathcal{S}_{\mathcal{B}_{4}}$

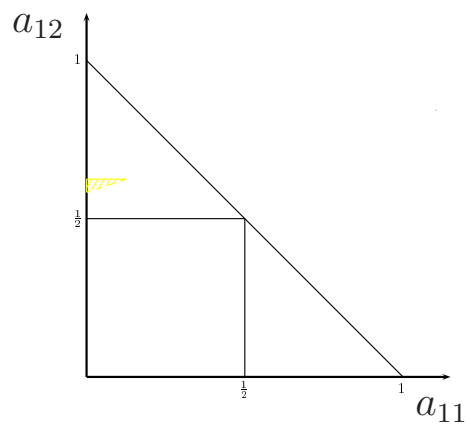

Feasible set $\mathcal{S}_{\mathcal{B}_{5}}$

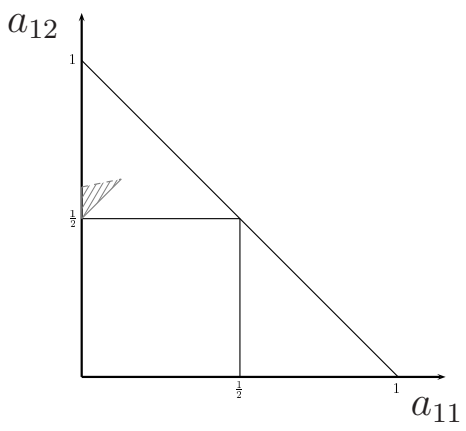

Feasible set $\mathcal{S}_{\mathcal{B}_{6}}$

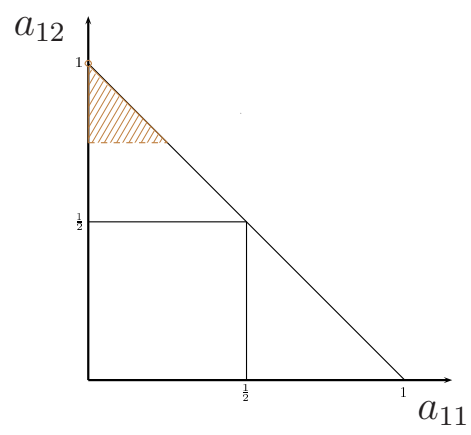

Feasible set $\mathcal{S}_{\mathcal{B}_{7}}$

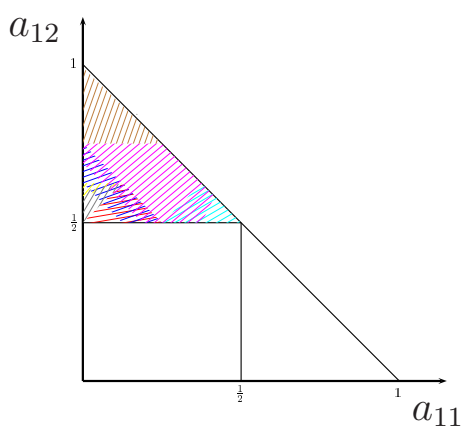

Feasible sets $\mathcal{S}_{\mathcal{B}_{1}}-\mathcal{S}_{\mathcal{B}_{7}}$

Figure 2: Feasible set $\mathcal{S}_{\mathcal{B}}$. 


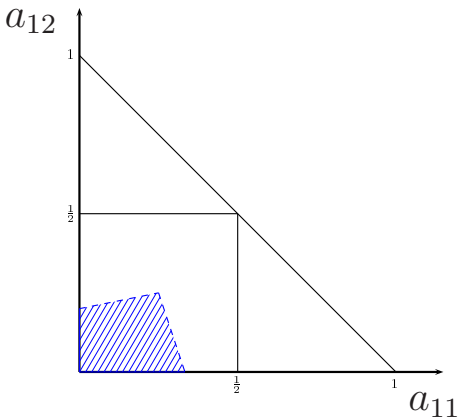

Feasible set $\mathcal{S}_{\mathcal{C}_{1}}$

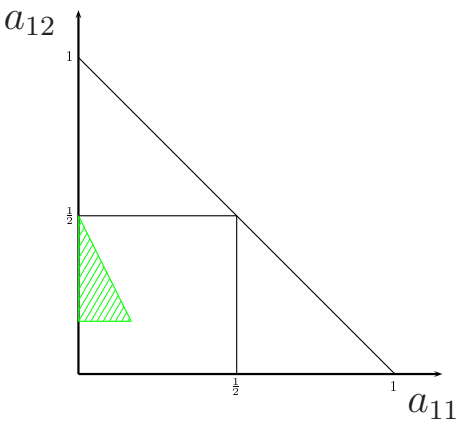

Feasible set $\mathcal{S}_{\mathcal{C}_{3}}$

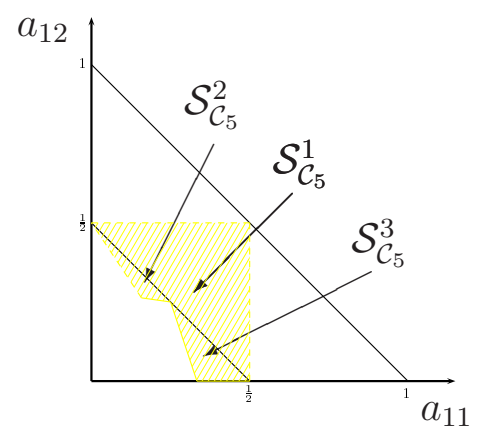

Feasible set $\mathcal{S}_{\mathcal{C}_{5}}$

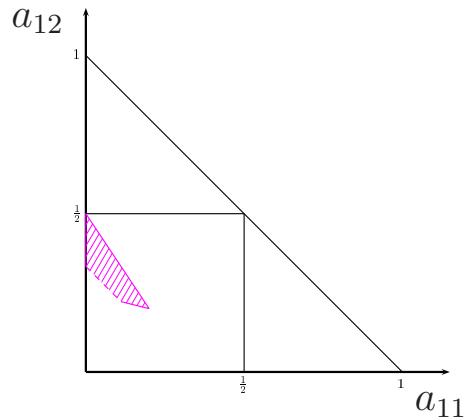

Feasible set $\mathcal{S}_{\mathcal{C}_{2}}$

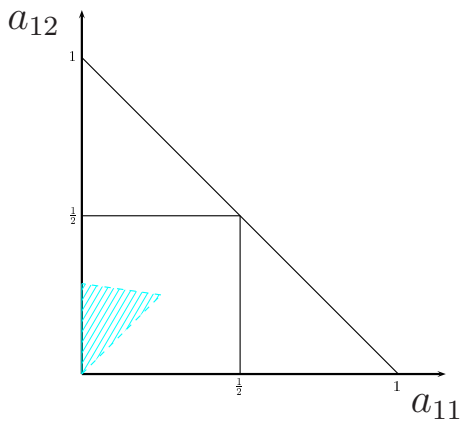

Feasible set $\mathcal{S}_{\mathcal{C}_{4}}$

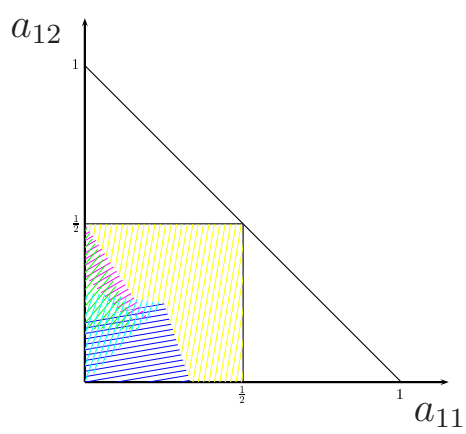

Feasible set $\mathcal{S}_{\mathcal{C}_{1}}-\mathcal{S}_{\mathcal{C}_{5}}$

Figure 3: Feasible set $\mathcal{S}_{\mathcal{C}}$. 


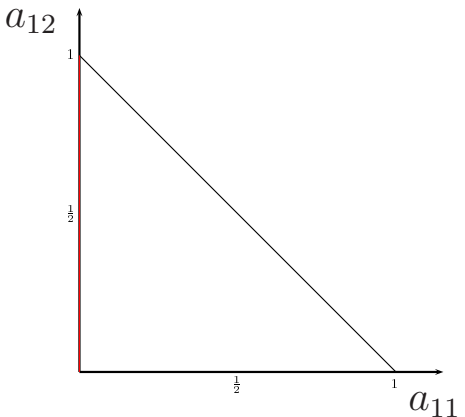

Feasible set $\mathcal{S}_{1}$

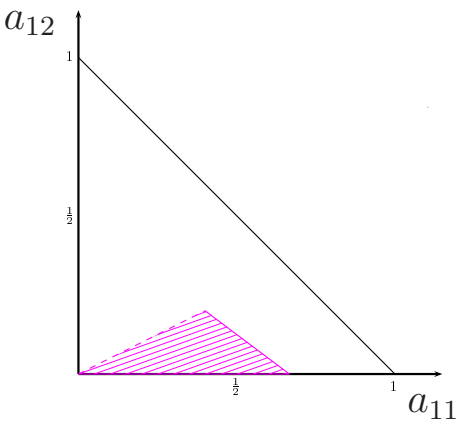

Feasible set $\mathcal{S}_{4}$

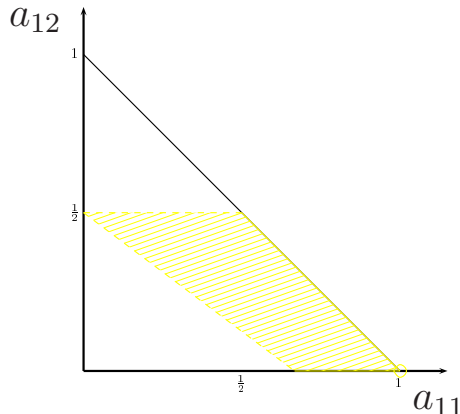

Feasible set $\mathcal{S}_{2}$

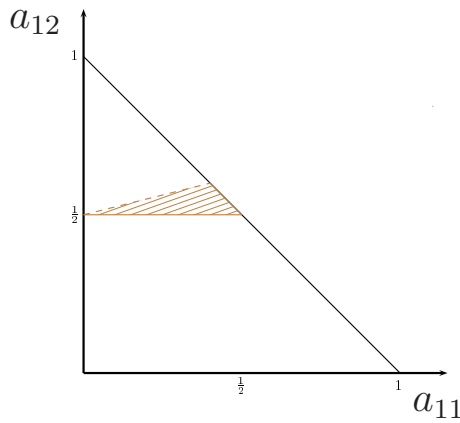

Feasible set $\mathcal{S}_{5}$

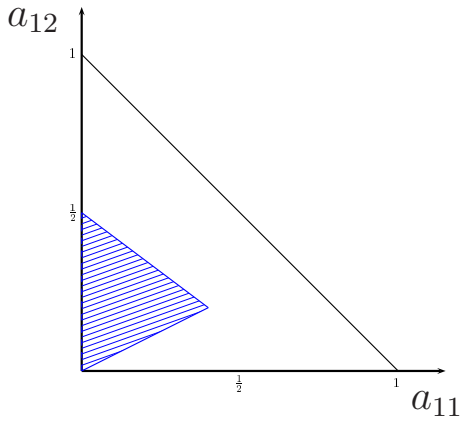

Feasible set $\mathcal{S}_{3}$

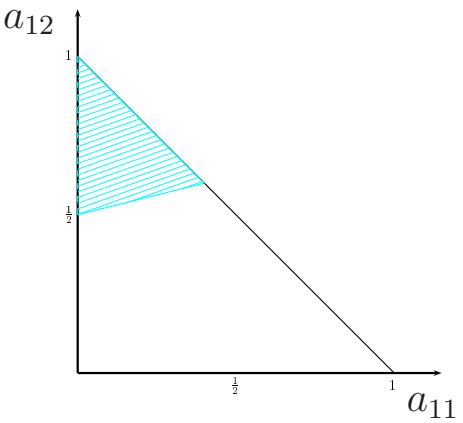

Feasible sets $\mathcal{S}_{6}$

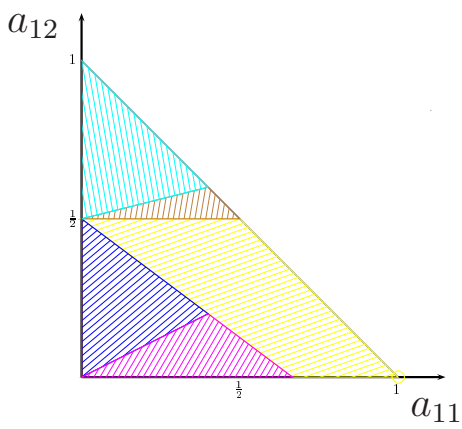

Feasible set $\mathcal{S}_{1}-\mathcal{S}_{6}$

Figure 4: Feasible set $\mathcal{S}$. 


\section{CESifo Working Paper Series}

(for full list see www.cesifo-group.de)

1812 Robert S. Chirinko and Hisham Foad, Noise vs. News in Equity Returns, September 2006

1813 Oliver Huelsewig, Eric Mayer and Timo Wollmershaeuser, Bank Behavior and the Cost Channel of Monetary Transmission, September 2006

1814 Michael S. Michael, Are Migration Policies that Induce Skilled (Unskilled) Migration Beneficial (Harmful) for the Host Country?, September 2006

1815 Eytan Sheshinski, Optimum Commodity Taxation in Pooling Equilibria, October 2006

1816 Gottfried Haber and Reinhard Neck, Sustainability of Austrian Public Debt: A Political Economy Perspective, October 2006

1817 Thiess Buettner, Michael Overesch, Ulrich Schreiber and Georg Wamser, The Impact of Thin-Capitalization Rules on Multinationals' Financing and Investment Decisions, October 2006

1818 Eric O’N. Fisher and Sharon L. May, Relativity in Trade Theory: Towards a Solution to the Mystery of Missing Trade, October 2006

1819 Junichi Minagawa and Thorsten Upmann, Labor Supply and the Demand for Child Care: An Intertemporal Approach, October 2006

1820 Jan K. Brueckner and Raquel Girvin, Airport Noise Regulation, Airline Service Quality, and Social Welfare, October 2006

1821 Sijbren Cnossen, Alcohol Taxation and Regulation in the European Union, October 2006

1822 Frederick van der Ploeg, Sustainable Social Spending in a Greying Economy with Stagnant Public Services: Baumol’s Cost Disease Revisited, October 2006

1823 Steven Brakman, Harry Garretsen and Charles van Marrewijk, Cross-Border Mergers \& Acquisitions: The Facts as a Guide for International Economics, October 2006

1824 J. Atsu Amegashie, A Psychological Game with Interdependent Preference Types, October 2006

1825 Kurt R. Brekke, Ingrid Koenigbauer and Odd Rune Straume, Reference Pricing of Pharmaceuticals, October 2006

1826 Sean Holly, M. Hashem Pesaran and Takashi Yamagata, A Spatio-Temporal Model of House Prices in the US, October 2006 
1827 Margarita Katsimi and Thomas Moutos, Inequality and the US Import Demand Function, October 2006

1828 Eytan Sheshinski, Longevity and Aggregate Savings, October 2006

1829 Momi Dahan and Udi Nisan, Low Take-up Rates: The Role of Information, October 2006

1830 Dieter Urban, Multilateral Investment Agreement in a Political Equilibrium, October 2006

1831 Jan Bouckaert and Hans Degryse, Opt In Versus Opt Out: A Free-Entry Analysis of Privacy Policies, October 2006

1832 Wolfram F. Richter, Taxing Human Capital Efficiently: The Double Dividend of Taxing Non-qualified Labour more Heavily than Qualified Labour, October 2006

1833 Alberto Chong and Mark Gradstein, Who's Afraid of Foreign Aid? The Donors' Perspective, October 2006

1834 Dirk Schindler, Optimal Income Taxation with a Risky Asset - The Triple Income Tax, October 2006

1835 Andy Snell and Jonathan P. Thomas, Labour Contracts, Equal Treatment and WageUnemployment Dynamics, October 2006

1836 Peter Backé and Cezary Wójcik, Catching-up and Credit Booms in Central and Eastern European EU Member States and Acceding Countries: An Interpretation within the New Neoclassical Synthesis Framework, October 2006

1837 Lars P. Feld, Justina A.V. Fischer and Gebhard Kirchgaessner, The Effect of Direct Democracy on Income Redistribution: Evidence for Switzerland, October 2006

1838 Michael Rauscher, Voluntary Emission Reductions, Social Rewards, and Environmental Policy, November 2006

1839 Vincent Vicard, Trade, Conflicts, and Political Integration: the Regional Interplays, November 2006

1840 Erkki Koskela and Mikko Puhakka, Stability and Dynamics in an Overlapping Generations Economy under Flexible Wage Negotiation and Capital Accumulation, November 2006

1841 Thiess Buettner, Michael Overesch, Ulrich Schreiber and Georg Wamser, Taxation and Capital Structure Choice - Evidence from a Panel of German Multinationals, November 2006

1842 Guglielmo Maria Caporale and Alexandros Kontonikas, The Euro and Inflation Uncertainty in the European Monetary Union, November 2006 
1843 Jan K. Brueckner and Ann G. Largey, Social Interaction and Urban Sprawl, November 2006

1844 Eytan Sheshinski, Differentiated Annuities in a Pooling Equilibrium, November 2006

1845 Marc Suhrcke and Dieter Urban, Are Cardiovascular Diseases Bad for Economic Growth?, November 2006

1846 Sam Bucovetsky and Andreas Haufler, Preferential Tax Regimes with Asymmetric Countries, November 2006

1847 Luca Anderlini, Leonardo Felli and Andrew Postlewaite, Should Courts always Enforce what Contracting Parties Write?, November 2006

1848 Katharina Sailer, Searching the eBay Marketplace, November 2006

1849 Paul De Grauwe and Pablo Rovira Kaltwasser, A Behavioral Finance Model of the Exchange Rate with Many Forecasting Rules, November 2006

1850 Doina Maria Radulescu and Michael Stimmelmayr, ACE vs. CBIT: Which is Better for Investment and Welfare?, November 2006

1851 Guglielmo Maria Caporale and Mario Cerrato, Black Market and Official Exchange Rates: Long-Run Equilibrium and Short-Run Dynamics, November 2006

1852 Luca Anderlini, Leonardo Felli and Andrew Postlewaite, Active Courts and Menu Contracts, November 2006

1853 Andreas Haufler, Alexander Klemm and Guttorm Schjelderup, Economic Integration and Redistributive Taxation: A Simple Model with Ambiguous Results, November 2006

1854 S. Brock Blomberg, Thomas DeLeire and Gregory D. Hess, The (After) Life-Cycle Theory of Religious Contributions, November 2006

1855 Albert Solé-Ollé and Pilar Sorribas-Navarro, The Effects of Partisan Alignment on the Allocation of Intergovernmental Transfers. Differences-in-Differences Estimates for Spain, November 2006

1856 Biswa N. Bhattacharyay, Understanding the Latest Wave and Future Shape of Regional Trade and Cooperation Agreements in Asia, November 2006

1857 Matz Dahlberg, Eva Mörk, Jørn Rattsø and Hanna Ågren, Using a Discontinuous Grant to Identify the Effect of Grants on Local Taxes and Spending, November 2006

1858 Ernesto Crivelli and Klaas Staal, Size and Soft Budget Constraints, November 2006

1859 Jens Brøchner, Jesper Jensen, Patrik Svensson and Peter Birch Sørensen, The Dilemmas of Tax Coordination in the Enlarged European Union, November 2006 
1860 Marcel Gérard, Reforming the Taxation of Multijurisdictional Enterprises in Europe, "Coopetition" in a Bottom-up Federation, November 2006

1861 Frank Blasch and Alfons J. Weichenrieder, When Taxation Changes the Course of the Year - Fiscal Year Adjustments and the German Tax Reform 2000/2001, November 2006

1862 Hans Jarle Kind, Tore Nilssen and Lars Sørgard, Competition for Viewers and Advertisers in a TV Oligopoly, November 2006

1863 Bart Cockx, Stéphane Robin and Christian Goebel, Income Support Policies for PartTime Workers: A Stepping-Stone to Regular Jobs? An Application to Young LongTerm Unemployed Women in Belgium, December 2006

1864 Sascha O. Becker and Marc-Andreas Muendler, The Effect of FDI on Job Separation, December 2006

1865 Christos Kotsogiannis and Robert Schwager, Fiscal Equalization and Yardstick Competition, December 2006

1866 Mikael Carlsson, Stefan Eriksson and Nils Gottfries, Testing Theories of Job Creation: Does Supply Create Its Own Demand?, December 2006

1867 Jacques H. Drèze, Charles Figuières and Jean Hindriks, Voluntary Matching Grants Can Forestall Social Dumping, December 2006

1868 Thomas Eichner and Marco Runkel, Corporate Income Taxation of Multinationals and Unemployment, December 2006

1869 Balázs Égert, Central Bank Interventions, Communication and Interest Rate Policy in Emerging European Economies, December 2006

1870 John Geweke, Joel Horowitz and M. Hashem Pesaran, Econometrics: A Bird's Eye View, December 2006

1871 Hans Jarle Kind, Marko Koethenbuerger and Guttorm Schjelderup, Taxation in TwoSided Markets, December 2006

1872 Hans Gersbach and Bernhard Pachl, Cake Division by Majority Decision, December 2006 